\title{
Optimal PEEP settings in Mechanical Ventilation using EIT
}

\author{
by \\ Ravi Baldev Govindgi Bhanabhai, B.Eng, Ryerson University
}

\begin{abstract}
A thesis submitted to the
Faculty of Graduate Studies and Postdoctoral Affairs

in partial fulfillment of the requirements for the degree of

Master of Applied Science in Electrical and Computer Engineering

Ottawa-Carleton Institute for Electrical and Computer Engineering

Department of Systems and Computer Engineering

Carleton University

Ottawa, Ontario, Canada, K1S 5B6
\end{abstract}

(C) Copyright 2012

Ravi B. G. Bhanabhai, April 2012 
Library and Archives

Canada

Published Heritage

Branch

395 Wellington Street

Ottawa ON K1A ON4

Canada
Bibliothèque et

Archives Canada

Direction du

Patrimoine de l'édition

395 , rue Wellington

Ottawa ON K1A ON4

Canada
Your file Votre référence

ISBN: 978-0-494-91577-6

Our file Notre référence

ISBN: $978-0-494-91577-6$
NOTICE:

The author has granted a nonexclusive license allowing Library and Archives Canada to reproduce, publish, archive, preserve, conserve, communicate to the public by telecommunication or on the Internet, loan, distrbute and sell theses worldwide, for commercial or noncommercial purposes, in microform, paper, electronic and/or any other formats.

The author retains copyright ownership and moral rights in this thesis. Neither the thesis nor substantial extracts from it may be printed or otherwise reproduced without the author's permission.
AVIS:

L'auteur a accordé une licence non exclusive permettant à la Bibliothèque et Archives Canada de reproduire, publier, archiver, sauvegarder, conserver, transmettre au public par télécommunication ou par l'Internet, prêter, distribuer et vendre des thèses partout dans le monde, à des fins commerciales ou autres, sur support microforme, papier, électronique et/ou autres formats.

L'auteur conserve la propriété du droit d'auteur et des droits moraux qui protege cette thèse. $\mathrm{Ni}$ la thèse ni des extraits substantiels de celle-ci ne doivent être imprimés ou autrement reproduits sans son autorisation.
In compliance with the Canadian Privacy Act some supporting forms may have been removed from this thesis.

While these forms may be included in the document page count, their removal does not represent any loss of content from the thesis.
Conformément à la loi canadienne sur la protection de la vie privée, quelques formulaires secondaires ont été enlevés de cette thèse.

Bien que ces formulaires aient inclus dans la pagination, il n'y aura aucun contenu manquant. 


\section{Abstract}

Ventilator Induced Lung Injury (VILI) is a serious condition caused by sub-optimal settings of mechanical ventilation in Acute Lung Injury (ALI) patients. The main contributors to VILI are 1) cyclic opening and closing of collapsed lung tissue which occur at low pressure and 2) overdistension of lung tissue which occur at high pressures. The key clinical measure to reduce VILI is selecting an appropriate Positive-End Expiratory Pressure (PEEP) to make a balance between keeping lung units open while not overdistending them. Electrical Impedance Tomography (EIT) provides regional lung air volume information which promises to help improve clinical selection of PEEP. The goal of this thesis is to develop automated methods to analyse EIT data to select a PEEP value. A novel algorithm is proposed to: 1) locate regional inflection points (IP) using a linear spline method and 2) to classify lung tissue as Collapsed, Nor-

mal, or Overdistened using a Fuzzy Logic System and to suggest an Optimal PEEP. These algorithms were implemented, tested, and compared to previously suggested approaches, using a clinical database of ALI and healthy lung patients. 


\section{Acknowledgments}

I would like to take this opportunity to to thank my supervisor, Dr. Andy Adler, whose guidance and extensive knowledge made this thesis possible and an excellent experience. I would also like to acknowledge his interests in computer systems in which he provided us with excellent equipment to perform our calculations. In particular the server provided that had the following specifications: Intel Corporation 5520 chipset, 16 Intel Corporation Xeon 5500 processors running at $2.67 \mathrm{GHz}$ with 4 cores and $8 \mathrm{MB}$ of cache in each processor, and 64GB of RAM. Working on his sever was a real fun experience.I would also like to acknowledge my colleagues whose advice, friendship, and intellectual conversations helped me progress through my masters. Without their help and companionship the long hours within the lab would not have been possible. In addition to my colleagues I would like to extend a thank you to the Systems and Computer Engineering Technical Department and Administrative staff. Their help with my computer issues and paper work helped save invaluable time.

Lastly, I would like to thank my family. Words can not say how much help they have provided me throughout my career at Carleton University. Their presence alone helped me get through the darkest times of my masters. To them I raise a glass. 
To my family,

who were always there during

the lows and the highs 


\section{Contents}

Front Matter $\quad$ iii

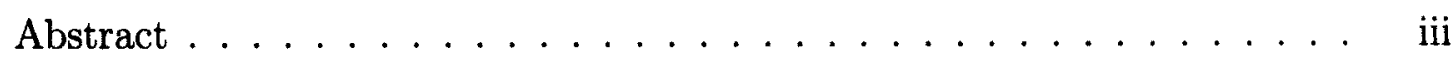

Acknowledgments ................... iv

Dedication . . . . . . . . . . . . . . .

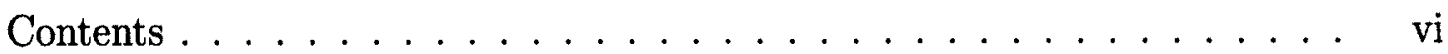

List of Figures . . . . . . . . . . . . . . $\mathrm{x}$



List of Acronyms \& Terminology $\ldots \ldots \ldots \ldots \ldots$ xvii

I Background 1

1 Introduction $\quad 2$

1.1 Thesis Objective $\ldots \ldots \ldots \ldots \ldots \ldots \ldots \ldots \ldots \ldots$

1.2 Thesis Contributions $\ldots \ldots \ldots \ldots \ldots \ldots$

2 Lung Injury $\quad 5$

2.1 Acute Lung Injury $\ldots \ldots \ldots \ldots \ldots \ldots \ldots \ldots$

2.2 Ventilated Induced Lung Injury $\ldots \ldots \ldots \ldots \ldots \ldots$

2.3 Pressure - Volume Curves $\ldots \ldots \ldots \ldots$ 
2.3.1 Models of Respiratory Function . . . . . . . . . . . 9

3 Electrical Impedance Tomography 12

3.1 EIT in Mechanical Ventilation . . . . . . . . . . . . . . . 13

4 Data and EIT Reconstruction $\quad 16$

4.1 Data . . . . . . . . . . . . . . . 16

4.1 .1 Patients . . . . . . . . . . . . . 17

4.1 .2 Pressure Maneuver . . . . . . . . . . . . . . 17

4.2 EIT Reconstruction . . . . . . . . . . . . . . . 18

4.2 .1 Forward Model . . . . . . . . . . . . . . . 22

4.2 .2 Reconstruction Model Used . . . . . . . . . . . . . . 23

4.2.3 Data pre-analysis . . . . . . . . . . . . . . 23

5 Fuzzy Logic $\quad 27$

5.1 Fuzzifier . . . . . . . . . . . . . . . . . . . 29

5.1 .1 Fuzzy Sets . . . . . . . . . . . . . . . . 29

5.1 .2 Membership Functions $\ldots \ldots \ldots \ldots$

5.2 Inference . . . . . . . . . . . . . . . . . 32

5.3 Defuzzifier . . . . . . . . . . . . . . . . 32

5.3 .1 Centroid Method . . . . . . . . . . . . . . 33

5.3 .2 Center-of-Sums . . . . . . . . . . . . . . . 33

5.3 .3 Height Defuzzifier . . . . . . . . . . . . . . . 33

5.3 .4 Center-of-Sets . . . . . . . . . . . . . . . . 34

II Contributions $\quad 35$

6 Inflection Points $\quad 36$ 
CONTENTS

6.1 What are Inflection Points . . . . . . . . . . . . 37

6.2 Importance of Inflection Points $\ldots \ldots \ldots \ldots \ldots \ldots$

6.3 Techniques to Locate Inflection Points $\ldots \ldots \ldots \ldots$

6.3 .1 Visual Heuristics $\ldots \ldots \ldots \ldots \ldots$

6.3 .2 Sigmoid Function $\ldots \ldots \ldots \ldots \ldots$

6.3.3 Three - Piece Linear Spline . . . . . . . . . . . . . . 43

6.4 Results . . . . . . . . . . . . . . . . . . . 44 44

6.5 Summary and Discussion . . . . . . . . . . . . . . 44

7 Algorithm Design $\quad 47$

7.1 Inflection Point Calculation $\ldots \ldots \ldots \ldots \ldots \ldots$



7.3 Premise Calculation . . . . . . . . . . . . . . . 53

7.4 Defuzzification . . . . . . . . . . . . . . . 54

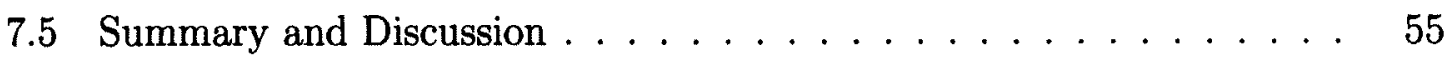

8 Results and Analysis $\quad 57$

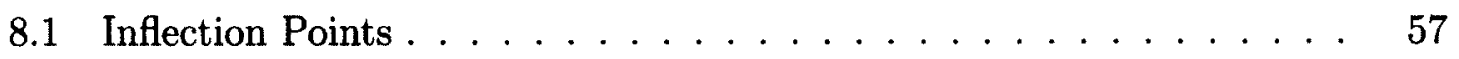

8.1.1 Inflection Points for Individual Algorithms . . . . . . . 57

8.1.2 Linear Spline vs. Sigmoid Method . . . . . . . . . . . 58

8.1.3 Linear and Sigmoid vs. Visual Heuristics . . . . . . . . . . 63

8.1 .4 Conclusion . . . . . . . . . . . . . . . 66

8.2 Membership Graph and IF-THEN Rules $\ldots \ldots \ldots \ldots$

8.3 Optimal PEEP $\ldots \ldots \ldots \ldots \ldots \ldots \ldots$

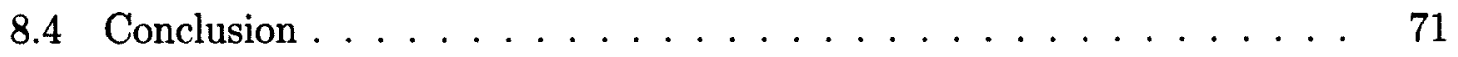

$\begin{array}{lll}9 & \text { Summary and Future Work } & 77\end{array}$ 
CONTENTS

9.1 Future Work . . . . . . . . . . . . . . . 78

9.1.1 Linear Spline Improvements ... . . . . . . . . . . 78

9.1.2 Fuzzy Logic Improvements . . . . . . . . . . . . . . . 79

9.1.3 Future Testing ................. 80

A MATLAB Code $\quad 82$

A.1 Linear Spline . . . . . . . . . . . . . . . . . . 82

A.1.1 Bin function needed for Spline . . . . . . . . . . 85

$\begin{array}{lr}\text { B Results Extra } & 89\end{array}$

B.1 Human vs. Linear Table . . . . . . . . . . . . . . . . . . 89

B.2 Visual Heuristics Pixel Locations . . . . . . . . . . . . . . . 90

$\begin{array}{ll}\text { References } & 91\end{array}$ 


\section{List of Figures}

2.1 An example of a pressure-volume and pressure-impedance curve for both inflation and deflation limbs. From these curves inflection points can be found to be used within guided ventilation strategies. For more information on inflection points refer to Chapter $6 \ldots \ldots \ldots$

4.1 Pressure, Volume, and Flow of the recruitment maneuver performed. Only the ramp regions, as segmented between the two lines was used for this study. The data before and after the black lines are of ventilation

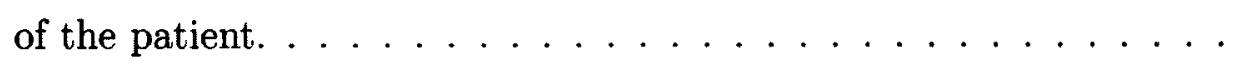

4.2 Location of the plane where the electrodes are placed equallyspaced around the patient, $5^{\text {th }}$ intercostal plane. Reproduced from [Rawlins et al., 2003] . . . . . . . . . . . . .

4.3 Example reconstructions using the GREIT methods of a healthy lung patient (patient 7). The outer most region in each image is from the reconstruction itself and has no physiological meaning. The second region from the outside in represents the chest and non-lung regions. The third and inner most region represents the lungs. Within the lung region the scale goes from light to dark, with the corresponding impedance going from high to low respectively. . . . . . . . . . 
4.4 Forward model based on adult human used to train GREIT with 16 electrodes. . . . . . . . . . . . . . . . . . .

4.5 Forward model used in the inverse solution for the data pre-analysis stage. . . . . . . . . . . . . . . . .

4.6 Pressure and EIT data aligned so peak is at time 0 demonstrating the relationship between the pressure ramp the measured impedance difference. In this image the impedance difference is for the entire lung. 26

5.1 Fuzzy Logic Design Schematic. Adapted from [Mendel, 2001] . . . . .

5.2 Triangle based membership function. Other types exist with this thesis using the trapezoidal function. . . . . . . . . . . .

6.1 Sigmoid function fit illustrating the location of each parameter. The data in this model was the same data used for the entire experiment. Each parameter is labeled and displays its value at optimization. $\mathrm{Pa}$ rameter $\mathrm{d}$ can be found using the value of 'c' the inflection points and $(6.2)$

6.2 Two examples of a sigmoid and linear spline fitted pressure-impedance curves with inflection points. The pressure-impedance data was selected from the indicated spot in the EIT lung image in the top left corner. It can be seen from the sigmoid graphs that most often both IP are not found, while the linear spline method is always able to locate

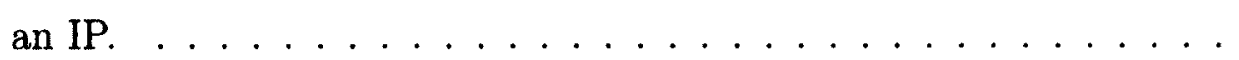


7.1 Example outputs from each stage of the algorithm. Starting with inflection point location the algorithm works toward fuzzification using the IP found the previous stage for the membership graphs. Once applying the rule base the premise is created and shown clearly. Finally each pixel is average and the PEEP is selected as shown in the last



7.2 Trapezoidal Fuzzy Membership graph. This is the type of membership graph used within the designed algorithm. . . . . . . . . .

8.1 How frequent each curve fitting method was able to locate an IP. The box around the median line represents the $75^{\text {th }}$ to $25^{\text {th }}$ quartile. The dashed lines outside of the box indicates the max and min values. . .

8.2 Difference in IP between the Sigmoid and Linear methods. The boxes around the median line represent the $75^{\text {th }}$ to $25^{\text {th }}$ quartile while the dashed line represents the max and min for each difference. A lack of comparative data is indicated in Patients with no quartile boxes. N/A signifies patients in which neither algorithm was able to locate an IP in the same pixel. . . . . . . . . . . . . .

8.3 Histogram of the minimum and maximum pressures for all patients. The maximum pressure for the inflation and deflation limb are the same. 61

8.4 Examples of both 'good' and 'bad' case scenarios encountered during the visual heuristic experiment. The PI data is taken from the pixel shown in the EIT image in the top left corner. . . . . . . . . . 
8.5 Box plot showing the difference in IP from the human heuristics method and the linear spline method. The boxes around the median line represents the $75^{\text {th }}$ to $25^{\text {th }}$ quartile while the dashed lines represents the $\max$ and $\min$ for each difference. $\ldots \ldots \ldots \ldots$

8.6 Box plot showing the difference in IP from the human heuristics method and the linear spline method. The boxes around the median line represents the $75^{\text {th }}$ to $25^{\text {th }}$ quartile while the dashed lines represents the max and min for each difference. A lack of comparative data is represented by no quartile box surrounding the median. N/A means the sigmoid method was unable to find an inflection points for any of the four pixels. $\ldots \ldots \ldots \ldots \ldots \ldots \ldots$

8.7 The progressive change of lungs regions as the pressure increases and decreases. The classifications are derived from the fuzzy logic system and show the progressive change from collapse to overdistended as the pressure increases. This figure also demonstrates the heterogeneous nature of the lungs. . . . . . . . . . . . . . .

8.8 Difference and average of the optimal PEEP found between $F S_{p}$ and $F S_{e} \ldots \ldots \ldots \ldots \ldots \ldots \ldots \ldots$

8.9 Difference and average of the optimal PEEP for both FLS and [Takeuchi et al., 2002] suggested PEEP of using LIP +2 mbar. Within Fig. $8.9 \mathrm{~b}$ the circles indicate further analysis was performed. . . . .

8.10 Difference between the human heuristic method and the linear spline method for the lower inflection point on the inflation limb only. . .

8.11 Global PI curve with LIP, UIP, and the LIP+2 mbar pressure and the Fuzzy optimal selection with according FLS based pressure. . . . . 
8.12 The PEEP suggested using both Pressure based and EIT based systems. Each data point represents a patient. From the figure it can be seen the inflation limb suggested PEEP is lower compared to respective deflation suggested PEEP. . . . . . . . . . . . . . . 76

9.1 Algorithm improvement Tree Chart . . . . . . . . . . . . . . 78

B.1 Locations of the pixels used in the visual heuristic experiment. . . . . 90 


\section{List of Tables}

4.1 Initial inverse model settings used for pre-analysis stage of the data. . 24

5.1 Fuzzy Operators $\ldots \ldots \ldots \ldots \ldots \ldots \ldots \ldots$

7.1 Details on creating the trapezoidal based fuzzy membership functions 52

7.2 Rule base table for the first and second fuzzy systems . . . . . . . 54

8.1 Mean, standard deviation, and median difference between the sigmoid and linear inflection points averaged over all patients. The units are in

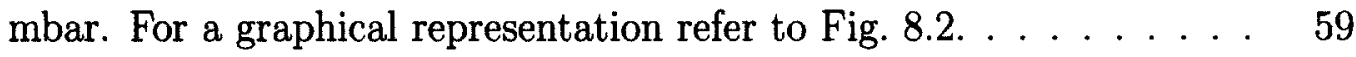

8.2 Correlations used to explain large Inflection Point difference between the Sigmoid and Linear method . . . . . . . . . . . . . . . 62

8.3 [Wu and Mendel, 2011] criteria for testing IF-THEN systems. $\ldots . .67$

8.4 Results of the difference comparison for the optimal PEEP between Fuzzy Rule System $\mathrm{p}$ and $\mathrm{e} \ldots \ldots \ldots \ldots$

8.5 Results of the comparison between Fuzzy Rule system p and e with [Takeuchi et al., 2002] result of LIP +2 mbar as a good PEEP. . . . 71 
B.1 Results from the comparison between the visual heuristics and the three piece linear methods. The table is formatted such that the first values indicate the median difference followed by it's standard deviation for the lower inflection points and then the median difference followed by it's standard deviation for the upper inflection point all for pixel 1. From here the pattern repeats for the last three pixels. . . . . 


\title{
List of Acronyms \& Terminology
}

\author{
ALI Acute Lung Injury \\ ARDS Acute Respiratory Distress Syndrome \\ CT Computed Tomography \\ EIDORS Electrical Impedance and Diffuse Optics Reconstruction Software \\ EIT Electrical Impedance Tomography \\ FEM Finite Element Method \\ FLS Fuzzy Logic System \\ GREIT Graz consensus Reconstruction algorithm for EIT \\ IP Inflection Points \\ LIP Lower Inflection Point \\ PAOP Pulmonary Artery Occlusion Pressure \\ PEEP Positive End-Expiration Pressure \\ PI Pressure-Impedance \\ PV Pressure-Volume \\ UIP Upper Inflection Point \\ VILI Ventilator Induced Lung Injury
}




\section{Part I}

\section{Background}




\section{Chapter 1}

\section{Introduction}

Ventilator Induced Lung Injury (VILI) is a serious condition associated with suboptimal settings within mechanical ventilation of Acute Lung Injury (ALI) patients. Its main causes are: 1) cyclical opening and closing of collapsed lung tissue occurring at low pressures and 2) overdistension of lung tissue occurring at high pressure. The purpose of this thesis was to investigate the use of Electrical Impedance Tomography (EIT) as a technology to help reduce VILI. Automated algorithms to process EIT data and make clinical assessments were developed and retrospectively tested.

EIT is an impedance based, non-invasive, and non-ionizing modality. It estimates the impedance distribution within a medium using electrical stimulation and voltage recordings at surface electrodes. This technique is relatively inexpensive and is an easy-to-use medical device for continuous bedside usage. It has medical applications in the fields of monitoring of pulmonary and cardiac functions, measurement of brain function, detection of hemorrhages, measurement of gastric imaging, detection and classification of tumors in breast tissue, and functional imaging of the thorax [Holder, 2004]. This thesis focused on the functional imaging of the thorax.

Using EIT, clinicians are now able to record the functional behavior of the lungs 
during a mechanical ventilation recruitment maneuver. EIT thus provides information for regional behavior and regional pressure-volume relations. It has been shown that air distribution within the lungs is heterogeneous [Grychtol et al., 2009, Harris, 2005, Andersen, 2008, Hasan, 2010] giving rise to erroneous recordings when considering global measurements. This thesis examines the use of EIT to categorize regions as collapsed, normal or overdistended which can then be used to suggest pressure settings to reduce VILI [Borges et al., 2006, Venegas et al., 1998, Takeuchi et al., 2002].

\subsection{Thesis Objective}

This thesis proposes a new method for the selection of mechanical ventilation settings from EIT data. The objective was split into two separate sections. The first was to use the EIT data along with pressure data, taken from the ventilation protocol, to locate regional inflection points from the pressure-impedance graphs. Such graph and inflection points can be seen in Fig. 6.2. The second was to create an automated system to use the fitted data to classify the lung regions as normal, overdistended, or collapsed. The algorithm is described in Chapter 7.

\subsection{Thesis Contributions}

This thesis looks into the use of a new technique for the location of regional inflection points (IP) from a short-time based low-flow recruitment maneuver. The regional inflection points are then used in a novel way within a Fuzzy Logic System (FLS) to produce a suggested pressure. The thesis contributions are divided into three separate sections and are listed below.

T-1 Summarize scholarly papers on acute respiratory distress syndrome (ARDS) - 
Reading and summarization of numerous papers on the topics ARDS, acute lung injury (ALI) and mechanical ventilation for a single source of knowledge on this topic.

T-2 EIT based methodology feature extraction - Using EIT and its associated regional data on the air movement in lungs, inflection points are located using a piece wise linear regression technique.

T-3 Using the IP obtained earlier a Fuzzy Logic System (FLS) was designed to classify the regions as being in healthy or injured. The location of the suggested pressure was found by maximizing the healthy states while minimizing injured states. 


\section{Chapter 2}

\section{Lung Injury}

This chapter is broken into three descriptive sections. The first being a description of Acute Lung Injury (ALI), the second of Ventilator Induced Lung Injury (VILI), and the third a description of Pressure-Volume (PV) curves. This overview of lung disease and clinical assessment tools motivates the technical work of further chapters in this thesis.

The lung is an essential organ within the respiratory system with its main function being the transport of oxygen into the bloodstream and moving carbon dioxide out of the bloodstream. Respiratory failure is a medical condition where the patient is unable to adequately control blood-gases transactions. It can come on abruptly as seen with acute respiratory failure or slowly as seen with chronic respiratory failure. Typically, respiratory failure initially affects the transfer of oxygen to the blood or the removal of carbon dioxide from the blood [Schraufnagel, 2010]. Oxygenation Failure usually is a sign of ALI and is discussed in this thesis. For further information on Ventilatory Failure (failure to remove carbon dioxide) refer to Chapter 20 from [Schraufnagel, 2010]. 


\subsection{Acute Lung Injury}

Acute Lung Injury is the umbrella term used to describe hypoxemic respiratory failure. ALI covers Acute Respiratory Distress Syndrome but also other milder degrees of lung injury [Schraufnagel, 2010]. ARDS is respiratory failure that results from widespread injury to the lungs and is characterized by fluid in the alveoli (pulmonary edema) with an abnormally high amount of protein in the edematous fluid and by hypoxemia [Merriam-Webster, 2010]. Two types of ARDS exist, the first is primary which is caused by direct injury and include inhalation based injury, near-drowning, and pneumonia. Secondary ARDS is caused by a chain of causation including burns and multiple blood transfusions [Hasan, 2010, Neligan, 2006].

The definition of ALI was clarified and categorized into four observations. [Hasan, 2010, Bernard et al., 1994].

1. Acute onset of respiratory failure (Minutes to Hours after injury)

2. Diffuse, bilateral pulmonary infiltrates on radiological images

3. Severe hypoxemia

4. Absence of a raised pulmonary artery occlusion pressure (PAOP)

Severe hypoxemia is quantified as the ratio of PAOP over inspired oxygen concentration $\left(\frac{\mathrm{PaO}_{2}}{\mathrm{~F}_{\mathrm{I}} \mathrm{O}_{2}}\right)$. Peer defined thresholds for ALI and ARDS have been defined as $266 \leq \frac{\mathrm{PaO}_{2}}{\mathrm{~F}_{1} \mathrm{O}_{2}} \leq 400 \mathrm{mbar}$ for ALI and $\frac{\mathrm{PaO}_{2}}{\mathrm{~F}_{1} \mathrm{O}_{2}} \leq 266 \mathrm{mbar}$ for ARDS. These thresholds help determine if a patient has undertaken severe hypoxemia or not. If the PAOP is $>24$ mbar it is considered raised thus anything less would satisfy criteria 4 [Hasan, 2010].

Normal alveoli have an inner layer of surfactant which helps to keep the lung tissue open during expiration, in diseased lungs this surfactant is lacking making the alveoli 
unstable and prone to collapse. When diseased lung tissue are repeatedly open and closed, shear stresses are generated. The shear stresses can produce ruptures causing damage [Hasan, 2010].

To treat ALI mechanical ventilation systems are used to alleviate the work of breathing. Either pressure controlled or volume controlled systems are used. A crucial support strategy is to set and maintain the Positive-End Expiratory Pressure (PEEP). PEEP is support pressure during exhalation to help maintain open lung regions leading to better oxygenation and reduction of cyclic opening and closing of lung tissue. Normal selection of PEEP is done by an iterative procedure where PEEP is adjusted till an appropriate oxygenation is reached. Each step in PEEP usually takes around 15-20 minutes in order to let oxygenation reach steady state [Hasan, 2010]. Because of the heterogeneous nature of lung tissue in ALI/ARDS patients not all the alveoli are collapsed at the same time thus while some tissue are opening others can be overdistending lending to Ventilator Induced Lung Injury (VILI) [Hasan, 2010, Andersen, 2008].

\subsection{Ventilated Induced Lung Injury}

Ventilator Induced Lung Injury is when acute lung injury is worsened by the use of mechanical ventilation [Hasan, 2010]. VILI can be broken down into three separate categories [Andersen, 2008].

1. Barotrauma / Volutrauma - is the rupture of the lung tissue caused by high pressure or high volumes. This can cause air leakage into the interstitial space making it difficult to breath among other complications.

2. Atelectrauma - is the injury associated with cyclic opening and closing of col- 
lapsed alveoli and is commonly caused by lack of pressure or volume to maintain open alveoli.

3. Biotrauma - is the increase in pulmonary and systemic inflammatory mediators. This tends to be a major source of death in ARDS patients as the inflammatory mediators can lead to organ failure This tends to occur during cyclic opening and closing of lung units.

Injuries in ventilated ARDS patients can lead to further alveolar ruptures causing complications such as pneumothorax. This case tends to happen when excess airway pressures is used. Commonly referred to barotrauma or alveolar overdistension. In addition to overdistension injury from ventilation systems can be caused by cyclic opening and closing of lung units. This cyclic action releases cytokines, a protein transmitter released during inflammatory response. This can be remedied by keeping lung units open via PEEP thus reducing the opening and closing behavior but insurances must be made on the max pressure or volume to avoid overdistension which can lead to barotrauma [Neligan, 2006]. Another notable factor in ventilation related injury is the level of inspiration fraction of $O_{2}\left(F_{I} O_{2}\right)$. High $F_{I} O_{2}$ can cause lung regions to be vulnerable to collapse as the high level of oxygen gets rapidly absorbed into the blood stream [Neligan, 2006].

\subsection{Pressure - Volume Curves}

Pressure-volume curves were used in 1946 to measure the mechanical function of the respiratory system. They were first used to diagnose ALI/ARDS patients in 1976, since ALI patients would have different mechanical properties compared to healthy lung patients. The curves were used to diagnose the progress of ALI patients in 1984, 
and in 1998 it was used as a means to guide ventilation settings [Andersen, 2008]. An example of a pressure-volume (PV) curve with its associated pressure-impedance (PI) curve is shown in Fig. 2.1. From the PV/PI curves IP can be located for the use in guided ventilation strategies.
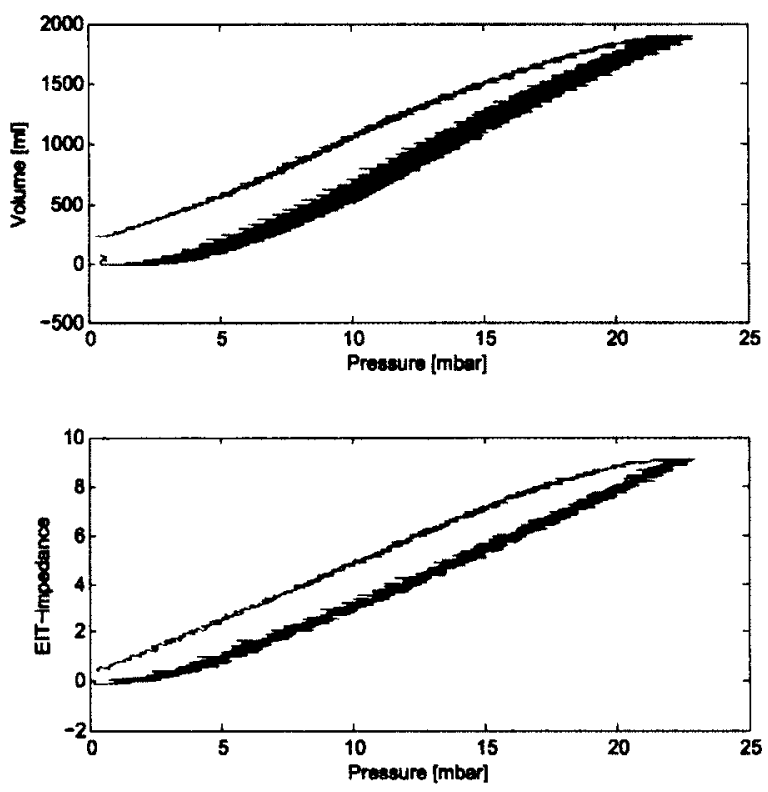

Figure 2.1: An example of a pressure-volume and pressure-impedance curve for both inflation and deflation limbs. From these curves inflection points can be found to be used within guided ventilation strategies. For more information on inflection points refer to Chapter 6

\subsubsection{Models of Respiratory Function}

There exist two main types of PV curves, static and dynamic. Both measure pressure and volume from the mouth but are performed differently and thus measure different physiological functions. (2.1) represents the relationship between airway pressure and volume where $P_{A O}$ is pressure at the airway opening, $V$ is the lung volume, $C$ is the respiratory system compliance, $\dot{V}$ is the gas flow, $R$ is the airway resistance, $\ddot{V}$ is the convective gas acceleration, $I$ is the impedance, and $P_{m u s}$ is the pressure generated from respiratory muscles [Harris, 2005]. 


$$
P_{A O}=\frac{V}{C}+\dot{V} R+\ddot{V} I-P_{m u s}
$$

Dynamic PV loops are obtained during a gas-flow intensive maneuver. From (2.1) we can see that dynamic PV curves will cause the resistive and impedance components to play a factor in the airway pressure. The respiratory muscles play a role in the airway pressure; thus, if the patient is inadequately sedated the recorded PV curve may be illustrating factors other then compliance. For the purpose of lung mechanics the compliance is of most importance as it represents ease of which lung tissue is ventilated so removal of the resistive, impedance, and respiratory muscles components are ideal. To remove the unwanted components from (2.1) static PV curves are used. Static PV curves use very slow airflow (near 0) causing the resistive component to be minimized. To reduce the impedance factors a constant airflow needs to be used causing the derivative to be zero. Removing the unnecessary components is beneficial as the resulting measurements will closely model the static compliance rather then the dynamic [Andersen, 2008, Hasan, 2010]. There exist three methods to acquire static PV curves.

1. Super syringe method is done by applying a syringe to the intubation tube and apply air in steps of 50 to $100 \mathrm{ml}$ till a max of $1.5 \mathrm{l}$ or 3.01 or 40 to 45 mbar of air have been applied. Between each step the air flow is ceased for 3 to 10 seconds to ensure static conditions are reached. The maneuver is slow and forces the patient to be disconnected from the ventilation system itself but also removes the resistive and impedance components of the respiration systems thus leaving the elastic components. The inflation maneuver alone takes between 45-60sec. [Andersen, 2008, Hasan, 2010, Lu et al., 1999].

2. Constant flow technique is a quasi-static method which does not require removal 
of the patient from the ventilation system. It performs a single inflation and deflation maneuver with constant flow all while taking continuous measurements of the pressure and volume. The slow flow is done to minimize the resistive component, with the constant property removing the impedance factor. Studies have shown that a flow $<9 \mathrm{~L} /$ min will suffice with larger flows producing right shifts in the PV curves when compared to static versions [Andersen, 2008, Harris, 2005, Hasan, 2010, Lu et al., 1999].

3. Multiple occlusion technique consists of various plateau volumes. At each plateau occlusion measurements are taken, once during inspiration and the second during expiration. Each measurement only takes 3 seconds and any volume decrease due to oxygen intake is considered negligible. Similar to the constant flow technique the patient does not need to be removed from the ventilation system but does require sedation to ensure no spontaneous breaths are taken during the procedure. The procedure lasts between 5 and 10 minutes [Andersen, 2008, Hasan, 2010, Lu et al., 1999].

For this thesis the [Pulletz et al., 2011] data was used which consisted of a slow constant flow maneuver with rates of $4 \mathrm{l} / \mathrm{min}$. Each patient was also sedated to remove the thorax muscle response. More details on the data and patients involved are listed in Chapter 4. 


\section{Chapter 3}

\section{Electrical Impedance Tomography}

EIT is an imaging modality that is non-invasive, non-ionizing and relatively inexpensive (thousands of dollars) [Boyle, 2010]. It produces a 2D conductivity image of a medium. With respect to imaging lung aeration the volumetric accuracy of an EIT system is within $10 \%$ of spirometric measurements [Holder, 2004]. EIT applies low frequency current $(50 \mathrm{kHz}$ and $\max 5 \mathrm{~mA})$ and measures the difference voltage through electrodes on the surface of the medium [Boyle, 2010, Holder, 2004]. The imaging system uses Finite Element Models to solve the forward problem where it simulates the voltage on the medium surface with known current and conductivity distribution of the medium. The reconstruction of EIT will be further discussed in Chapter 4. In Canada, EIT machines used with human patients must first comply with the International Electrotechnical Commission standard 60601-1 and must be reviewed by a certified laboratory like the Canadian Standards Association [Boyle, 2010]. The introduction of the EIT system for human studies occurred in the mid 1980s, with the first written book in 1990 [Holder, 2004]. Starting in the field of mineral exploration it moved to the biomedical domain in the early 1980s [Holder, 2004, Allud and Martin, 1977]. Measuring the conductivity of a medium 
EIT allows for a new look at the distribution of air, blood, and extravascular liquid within the lungs [Adler et al., 1997]. Having gone through rigorous validation, [Hinz et al., 2003b, Victorino et al., 2004, Gattinoni et al., 1987] EIT is now taking the next step into specific applications with focus on lung-air distributions.

\subsection{EIT in Mechanical Ventilation}

Using EIT to measure lung aeration provides increased measurement accuracy and allows for better PEEP selection for ventilation systems. This is of particular significance given that thoracic CT scans reveal heterogeneity of air distribution within a pathological lung [Gattinoni et al., 1987]. CT images are considered a gold standard for detailed images of the thorax but they require moving an unstable patients to the imaging area and expose the patient to ionizing radiation making it a poor choice for repeated bedside use. EIT on the other hand is non-ionizing and is capable of measuring the distribution of air within the thorax. For instance with a current input of $50 \mathrm{kHz}$ the resistivity of deflated lung tissue is 12.5 $\Omega \cdot m$ while inflated lung tissue has a resistivity of around $25.0 \Omega \cdot m$ [Holder, 2004]. With this large difference in resistivity between inflated and deflated lung tissue EIT has been recommended for regional lung monitoring [Hahn et al., 1996]. In the past global PV curves were used to locate IP and were used to set PEEP accordingly [Hinz et al., 2006, Papadakis and Lachmann, 2007]. It has come to knowledge that within ALI patients the distribution of air within the lungs is heterogeneous making global PV measurements too general to customize PEEP choices [Victorino et al., 2004]. EIT provides regional conductivity information and is able to produce pressure-impedance curves where pressure settings can be extracted. With all the advancements in this field much work still needs to be done by standardizing 
inflection point detection algorithms and features to use for classification systems.

The use of EIT in mechanical ventilation is still in a inchoate stages of development. Early studies using EIT in the lung domain focused primarily on validation [Hahn et al., 1996, Frerichs et al., 2002, Hinz et al., 2003a, Victorino et al., 2004]. Subsequent research worked toward extracting information, like inflection points and pressure-impedance curves, and drawing conclusions related to the state of lung tissue [Kunst et al., 2000, Gattinoni et al., 1987, Amato et al., 1998, Kunst et al., 1999, Genderingen et al., 2004, Adler et al., 2012]. More research is necessary to understand guided ventilation strategies, create automated systems, to determine accurate rule bases and incorporate uncertainty into the Fuzzy Logic Systems [Grychtol et al., 2010, Luepschen et al., 2007].

Research comparing global and regional PV curves shows that the former can be represented as a sum of the latter [Kunst et al., 2000]. Using PV curves to identify therapeutic pressure to reduce VILI has been confirmed. Chapter 6 further elaborates on the use of PV curves and inflection points. Furthermore, [Kunst et al., 2000, Kunst et al., 1999] noticed that dependent regions have higher LIP then the counter parts in the non-dependent region. The airs dependency on gravity increases collapsed regions in the direction of gravity. This gravitational dependency helps explain the continual recruitment along the linear portion of a global PV curve and the need for a regional bedside imaging systems like EIT.

The regional information provided by EIT clarifies the understanding of ventilation distributions, locating regions of overdistension and regions of collapse, one sided ventilation, regional compliance, LIP and UIP, and delays in ventilation [Adler et al., 2012]. The reconstruction system used for this thesis produced images of size $32 \times 32$ pixels. The average lung-region of interest (lung-ROI) for all patients was $248.88 \pm 53.33$ pixels. From Chapter 4 the average height for all patients was 
$177 \pm 9 \mathrm{~cm}$. Using the median relationship between external chest size and height from [Todd, 2010], which can be seen in (3.1), a max circumference of $105.4 \mathrm{~cm}$ and minimum circumference of $95.2 \mathrm{~cm}$ was found. This resulted in a resolution of $3.38 \pm 0.923 \mathrm{~cm}^{2}$ per pixel. The resolution value is an approximation due to the use of lung-region of interest and the relationship matching external chest size to height. Using the chest-region of interest of which the size is 575 pixels for all patients as a replacement for the lung-ROI a resolution of $1.39 \mathrm{~cm}^{2}$ per pixel is realized. It can then be argued that the resolution for this particular EIT system is between 1.39 and $3.38 \pm 0.923 \mathrm{~cm}^{2}$ per pixel.

$$
c i r_{\text {median }}=\frac{1}{2}(h e i g h t)+\frac{1}{15}(h e i g h t)
$$

Some research has been conducted to apply the regional information to create automated mechanical ventilation systems [Grychtol et al., 2010]. However, many gaps in the literature still exist. For example, determining which features should be extracted from the regional information collected is an area of research garnering further attention. With EIT as a bedside device options to have fast and reliable warning parameters can be available. Being able to detect collapsed and overdistended regions will allow for warnings to signal the physician or alternate the PEEP to reduce potential injuries [Adler et al., 2012].

Further research is necessary to improve the current use of EIT. Shortcomings of current EIT systems include 2D dependencies, errors associated with posture and diaphragm position, and the use of difference based EIT measurements [Adler et al., 2012]. 


\section{Chapter 4}

\section{Data and EIT Reconstruction}

This chapter describes the data and reconstruction algorithm used within this thesis. The first section covers the number of patients dividing them into ALI and control. It also covers the ventilation protocol along with the mechanical ventilation unit and the EIT system used in the measurements. Data pre-analysis is explained in the data section of this chapter and covers EIT-pressure-alignment and locating the start and end of the pressure ramp aka the recruitment maneuver.

In the second section the algorithm used to reconstruct the EIT data is described with focus on the parameters used, a description of the reconstruction, and the advanced nature of the algorithm.

\subsection{Data}

The data was obtained from [Pulletz et al., 2011] where a low-flow pressure based recruitment maneuver with synchronized EIT was performed. 


\subsubsection{Patients}

The data used for this thesis was of human trials from the medical university center at Schleswig Holstein campus in Kiel. The experiment received local ethics approval for 26 patients. The patients were taken from the surgical intensive care unit and the operations theaters with written consent provided from each patient or a legal representative. All of the patients within the study were sedated, paralyzed and artificially ventilated using a pressure controlled ventilation mode while in the supine position. The distribution of the patients consisted of 8 healthy lung patients (age: $41 \pm 14$ years, height: $177 \pm 8 \mathrm{~cm}$, weight: $76 \pm 8 \mathrm{~kg}$, mean $\pm \mathrm{SD}$ ) and 18 ALI patients, (age: $58 \pm 14$ years, height: $177 \pm 9 \mathrm{~cm}$, weight: $80 \pm 11 \mathrm{~kg}$ ) which fulfilled

the American-European consensus criteria for ALI (rapid onset, $\frac{P_{a} O_{2}}{F^{2} O_{2}} \leq 300 \mathrm{mmHg}$, bilateral infiltrates, and no clinical sign for left atrial hypertension.

\subsubsection{Pressure Maneuver}

A low flow inflation-deflation-pressure-volume maneuver was performed using an Evita XL (Draeger, Luebeck, Germany) mechanical ventilator. Prior to the ramp recruitment maneuver ventilation of the patient took place and can be seen in Fig. 4.1 as the oscillations before and after the ramp. The maneuver started at zero Positive End-Expiratory Pressure (PEEP) with a constant gas flow of $4 \mathrm{l} / \mathrm{min}$ and went up to a tidal volume of 21 or until a maximum airway pressure of 35 mbar. Fig. 4.1 illustrates a sample of the pressure, volume and volume flow used. The mechanical ventilator sampled the input data at a rate of $126 \mathrm{~Hz}$ and was synchronized to an EIT data acquisition system via USB with a sampling rate of $25 \mathrm{~Hz}$.

The EIT data was gathered using a GOE-MF II EIT (CareFusion, Hoechberg, Germany) system with 16 self-adhesive electrodes (Blue sensor L-00-S, Ambu, Ballerup, 

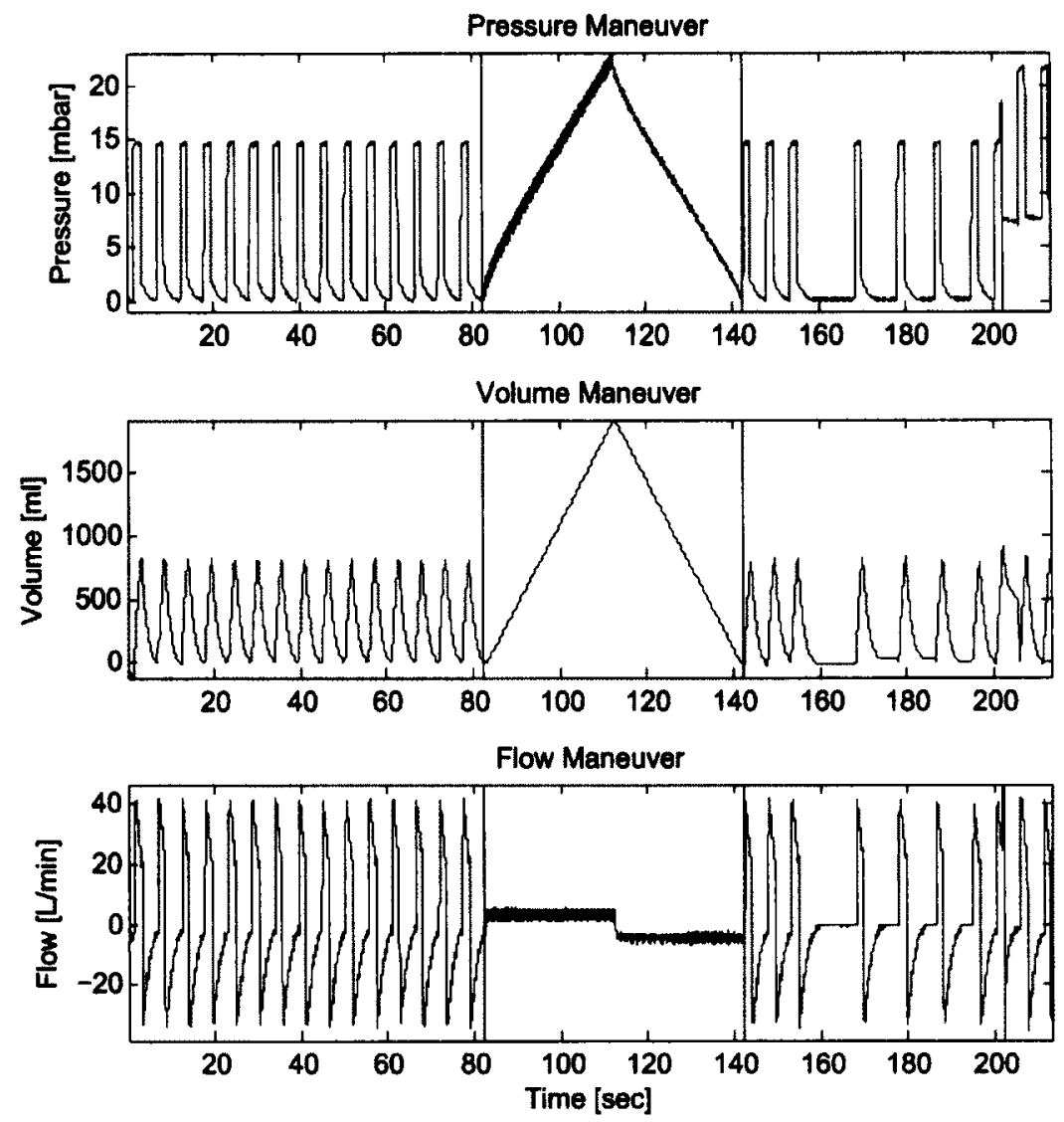

Figure 4.1: Pressure, Volume, and Flow of the recruitment maneuver performed. Only the ramp regions, as segmented between the two lines was used for this study. The data before and after the black lines are of ventilation of the patient.

Denmark). The electrodes were positioned approximately along the 5th intercostal plane around the patients chest as shown in Fig. $4.2 .50 \mathrm{kHz}$ and $5 \mathrm{~mA}_{\mathrm{rms}}$ electrical current was applied through an adjacent pair configuration with the remaining electrodes being used to measure the voltage after the current injection. The EIT data was acquired at a rate of 25 scans per second and processed offline.

\subsection{EIT Reconstruction}

For this thesis the Graz consensus Reconstruction algorithm for Electrical Impedance Tomography (GREIT) was used. Started in 2007 at the ICEBI conference in Graz, 


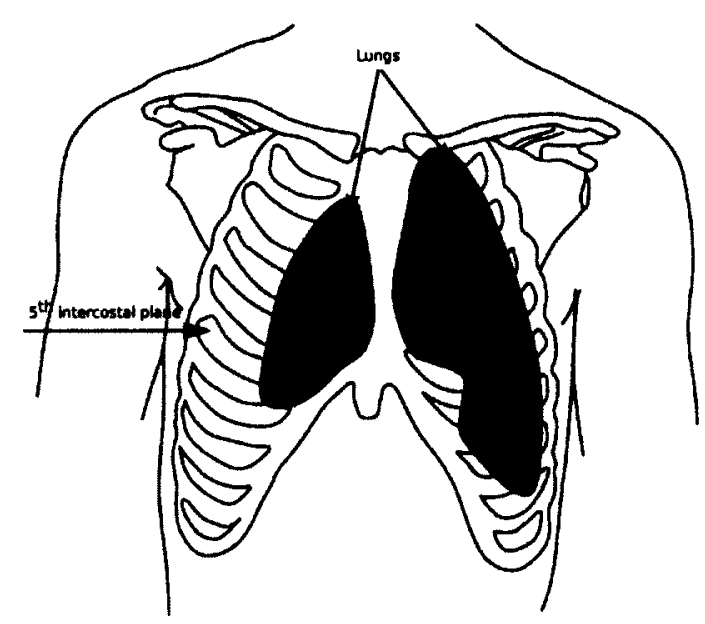

Figure 4.2: Location of the plane where the electrodes are placed equally-spaced around the patient, $5^{\text {th }}$ intercostal plane. Reproduced from [Rawlins et al., 2003]

Austria, GREIT came about as a way to draw a standard for linear EIT reconstruction. The work was done by researchers from a wide range of disciplines. GREIT was made to standardize the linear reconstruction of EIT images and does not consider calibration tests, data formats or phantoms, standards in image interpretation, or EIT base lung parameters. The following specifications are known not to be ideal but are rather a basis which to improve upon. GREIT suggests the following for an EIT system [Adler et al., 2009].

Use a single ring electrode configuration with a Sheffield-type EIT system using adjacent current injection and measurement. Linear (i.e. real-time) reconstruction of a $2 \mathrm{D}$ conductivity change image, based on a 3D forward model. Quantitative difference reconstruction for which units can be assigned to EIT images. Reconstruction onto a $32 \times 32$ pixel array for a single ring of 16,12 and 9 electrodes, for the shapes: a) neonatal chest, b) male and female adult chest, and c) cylindrical tank. [Adler et al., 2009]

GREIT adjusts the linear reconstruction to meet certain performance requirements. The performance requirements were found via consensus. In doing so the 
reconstruction algorithm modifies its minimization criteria thus not satisfying the underlying mathematical model. A linear reconstruction algorithm is used rather then an iterative approach as they tend to fail. The failure is brought upon by measurement noise and geometric uncertainty in clinical and experimental EIT data. A linear approach also facilitates real-time reconstruction.

The GREIT inverse model $\mathbf{R}$ differs depending on the choice of the forward model, noise model, and the desired performance metrics. The forward model allows calculations of voltage differences, $\mathbf{y}^{(\mathbf{k})}$, from a conductivity change, $\mathbf{x}^{(\mathbf{k})}$, where $\mathbf{k}$ represents the indices for the training set. The forward model provides the details of the body's geometry, the electrode size and contact impedance, and the reference conductivity $\left(\sigma_{\mathbf{r}}\right)$ around which conductivity changes occur. GREIT uses a 3D FEM forward model using the complete electrode model [Adler et al., 2009]. From the forward model the measurement variance is calculated.

For the noise model the measurement data from the forward model is used. In GREIT two sources of noise are considered: electronic measurement noise, and electrode movement artifacts. The electronic measurement noise can be modeled as various distributions but uniform Gaussian is acceptable for generic EIT reconstruction and was used. The measurement noise is usually related to the EIT hardware and patient connections such as different gain settings on each channel. Electrode movement artifacts happen when electrodes move with posture or chest movement during breathing and have reported to cause significant artifacts [Adler et al., 1996, Zhang and Patterson, 2005, Coulombé et al., 2005]. To reduce the noise, augmented forward models based on both the conductivity change and electrode movement can be made to reduce the artifacts. This is currently implemented by deformations of the FEM but can be based on a calibration protocol in an implemented system. 
Using the desired performance metrics, training is performed to find a well suited reconstruction matrix. This is done by using "desired images", $\tilde{\mathbf{x}}_{\mathbf{t}}^{(\mathbf{k})}$, in a training set of size $(k)$. The "desired images" correspond to locations of conductivity in the forward model, $\mathbf{x}_{\mathbf{t}}^{(\mathbf{k})}$. $\tilde{\mathbf{x}}_{\mathbf{t}}$ is centered at the same location as $\mathbf{x}_{\mathbf{t}}$ but is designed to be circular and have an accompanying blur radius around the center. The use of the desired image allows for uniform resolution throughout the entire image at the expense of lower resolution along the boundaries. For each "desired image" a weight image $\mathbf{w}^{(\mathbf{k})}$ is created to put weights to each pixel within the "desired image". These weights are used to put more emphasis on some metrics rather than others. For each "desired image" there exist two circular regions, the first is centered around the targeted position to emphasis a flat amplitude while the second regions is outside of the first and represents a region in which the amplitude is to be zero. In between the two regions the desired image is supposed to smoothly transition from the inner region amplitude to zero, the outer region. Larger weight is given to these regions to enforce that performance metrics are met while smaller weights are selected for the in transition region to allow the algorithm to have flexibility to meet other specifications.

In addition to the conductivity targets, noise training samples are used for electronic noise and movement artifacts. For noise training the desired image $\left(\tilde{\mathbf{x}}_{\mathbf{n}}^{(\mathbf{k})}\right)$ is zero. The weight image $\left(\mathbf{w}^{(\mathbf{k})}\right)$ is large to enforce a penalty on noise.

Based on the forward model, noise model, and desired performance an improved GREIT reconstruction matrix can be created by solving the minimization express for error $\epsilon^{2}$ as seen in (4.1). 


$$
\begin{aligned}
\epsilon^{2} & =\sum_{k}\left\|\tilde{\mathbf{x}}^{(\mathbf{k})}-\mathbf{R} \mathbf{y}^{(\mathbf{k})}\right\|_{\mathbf{w}^{(\mathbf{k})}}^{2} \\
& =\sum_{k} \sum_{i}\left(\left[\tilde{\mathbf{x}}^{(\mathbf{k})}\right]_{i}^{2}\left[\mathbf{w}^{(\mathbf{k})}\right]_{i}^{2}-2\left[\tilde{\mathbf{x}}^{(\mathbf{k})}\right]_{i}\left[\mathbf{w}^{(\mathbf{k})}\right]_{i}^{2}\left(\sum_{j} \mathbf{R}_{\mathbf{i j}}\left[\mathbf{y}^{(\mathbf{k})}\right]_{j}\right)\right. \\
& \left.+\left[\mathbf{w}^{(\mathbf{k})}\right]_{i}^{2}\left(\sum_{j} \mathbf{R}_{\mathbf{i j}}\left[\mathbf{y}^{(\mathbf{k})}\right]_{j}\right)^{2}\right)
\end{aligned}
$$

The sum $k$ is over all the training measurement and noise samples. $\mathbf{W}^{(\mathbf{k})}=$ (diag $\left.\mathbf{w}^{(\mathbf{k})}\right)^{2}$ is a diagonal matrix representing the weight corresponding to each measurement. From (4.1) an expression for $\mathbf{R}=\arg \min \epsilon^{2}$ is found by setting the derivative $\frac{\partial \epsilon^{2}}{\partial \mathbf{R}_{i j}}$ to zero. The math is worked through in [Adler et al., 2009] and was simplified to (4.2).

$$
=\mathbf{A}_{\mathbf{i j}}-\sum_{l} \mathbf{R}_{\mathbf{i l}} \mathbf{B}_{\mathbf{i j l}}
$$

Matrix $\mathbf{A} \in \mathbb{R}^{n_{N} \times n_{M}}$ and tensor $\mathbf{B} \in \mathbb{R}^{n_{N} \times n_{M} \times n_{M}}$ and are defined as $\mathbf{A}=$ $\sum_{k}\left[\tilde{\mathbf{x}}^{(\mathbf{k})}\right]_{i}\left[\mathbf{w}^{(\mathbf{k})}\right]_{i}^{2}\left[\mathbf{y}^{(\mathbf{k})}\right]_{j}$ and $\mathbf{B}=\sum_{k}\left[\mathbf{y}^{(\mathbf{k})}\right]_{l}\left[\mathbf{w}^{(\mathbf{k})}\right]_{i}^{2}\left[\mathbf{y}^{(\mathbf{k})}\right]_{j}$. If the weights, w, are kept the same for each training data set, (4.2) can be simplified to $\mathbf{A}=\mathbf{R}_{\mathbf{G B}} \mathbf{B}$ which gives the final GREIT reconstruction matrix of $\mathbf{R}_{\mathbf{G B}}=\mathbf{A B}^{-\mathbf{1}}$.

\subsubsection{Forward Model}

Creating the training data for the learning methods takes a forward model to map conductivity contrasts targets, $\mathbf{x}_{t}$ to difference measurements, $\mathbf{y}$. The forward models are built using a 3D first order tetrahedral finite element model and are solved using preconditioned linear solvers [Polydorides and Lionheart, 2002]. Finite element models which have been made are: male and female adult chests, a neonatal chest, 


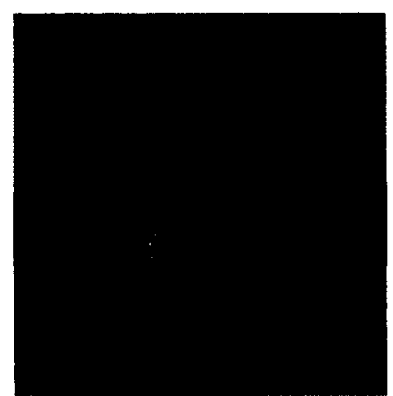

(a) Start of Inflation



(b) Max Pressure

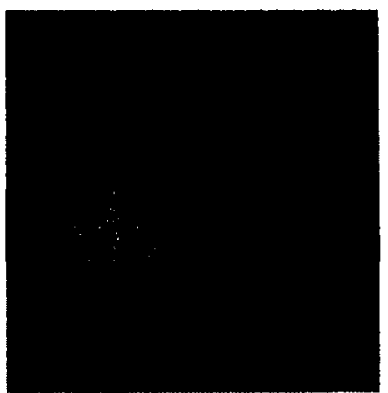

(c) End of Deflation

Figure 4.3: Example reconstructions using the GREIT methods of a healthy lung patient (patient 7). The outer most region in each image is from the reconstruction itself and has no physiological meaning. The second region from the outside in represents the chest and non-lung regions. The third and inner most region represents the lungs. Within the lung region the scale goes from light to dark, with the corresponding impedance going from high to low respectively.

and a cylinder. It is possible to create patient specific FEM which would provide a better reconstruction but as [Adler et al., 2009] has stated from experience working with time-difference EIT the four models provided work with most of the accuracy of an adaptive meshing. For this thesis the male human thorax FEM model was used.

\subsubsection{Reconstruction Model Used}

For this thesis the adult male model with 16 electrodes was used. The first electrode was placed on the median dorsal part of the model and the ninth electrode placed directly across. Fig. 4.4 shows both the 3D model as well as the $2 \mathrm{D}$ version with electrode placement used. The training was done using normalized measurements with 500 samples of $3 \%$ of the model diameter in size targets.

\subsubsection{Data pre-analysis}

All analysis was done offline with the majority of work being done in the Fuzzy Logic System FLS. This section explains the code designed by [Pulletz et al., 2011] and was 

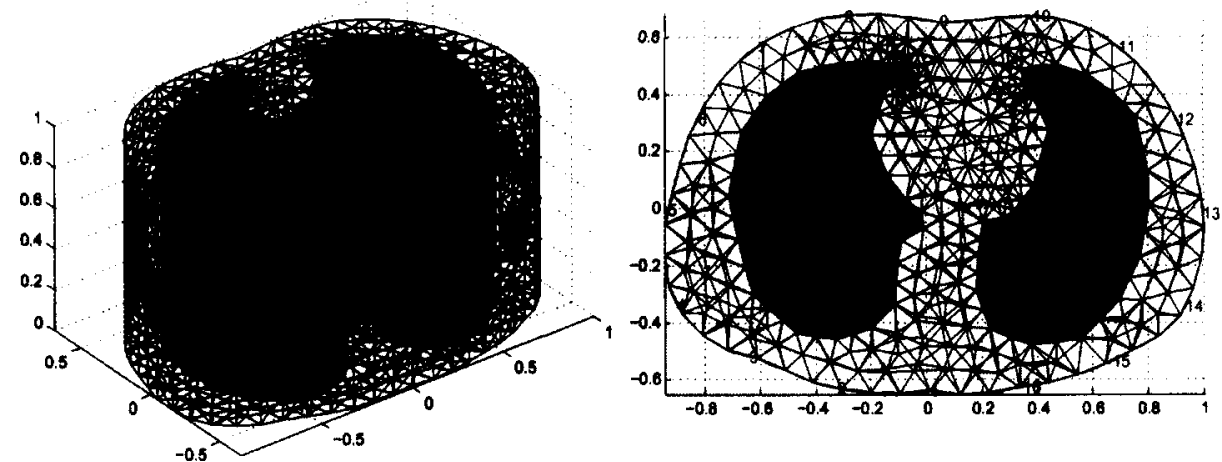

Figure 4.4: Forward model based on adult human used to train GREIT with 16 electrodes.

used to setup the input data to the algorithm explained in Chapter 7.

The first step in data preparation was to locate the start, max, and end points in the pressure-volume maneuver. This was done by first inputting the pressure and EIT data into the analysis program. From here the image was reconstructed using a $2 \mathrm{D}$ chest thorax forward model with 1024 elements, with reference being the mean of the entire voltage signal and normalized measurements set. Fig. 4.5 displays the forward model used. The software used to perform the inverse solution and forward model calculations was EIDORS [EIDORS, 2011]. The settings for the inverse model is displayed are Table 4.1 .

\begin{tabular}{|l|l|c|}
\hline \multicolumn{2}{|c|}{ Parameter } & Value \\
\hline \hline RtR_prior & $=$ & Image=LaPlace Prior \\
& & Movement=Smoothness Constraint \\
rm.aa_e_move_image_prior.parameters & $=$ & 0.1 \\
rm.hyperparameter.value & $=$ & 0.06 \\
rm.inv_solve.select_parameters & $=$ & $1: 1024$ \\
rm.aa_e_move_image_prior.RegC.func & $=$ & LaPlace Prior \\
\hline
\end{tabular}

Table 4.1: Initial inverse model settings used for pre-analysis stage of the data.

The EIT impedance values were then averaged over the Finite Element Model (FEM) element area. From here a time vector was created for the EIT and pressure signals using the sampling rate of $29 \mathrm{~Hz}$ and $126 \mathrm{~Hz}$ respectively. Both time signals 


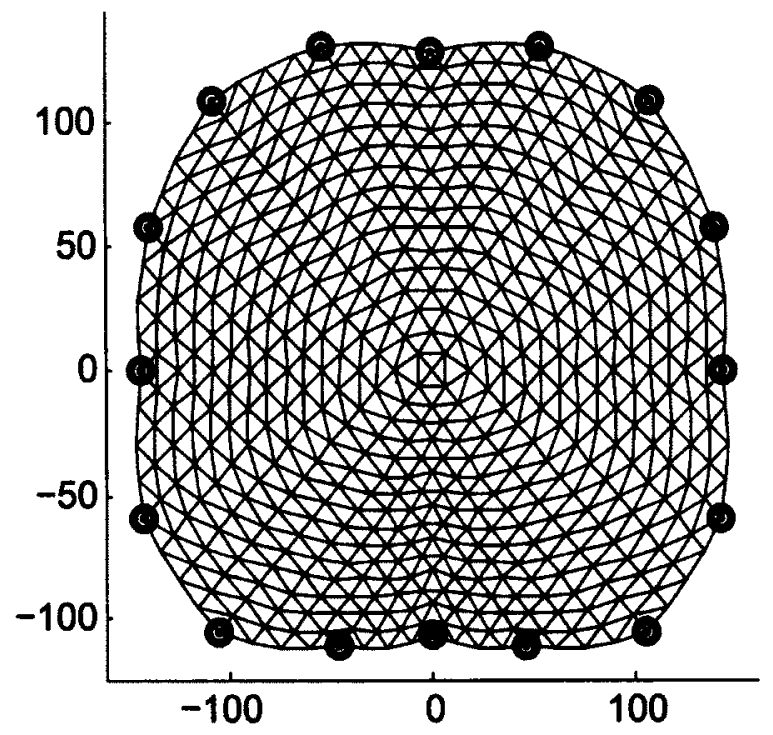

Figure 4.5: Forward model used in the inverse solution for the data pre-analysis stage.

were centered so that the max pressure / EIT values were situated at time index 0 as illustrated in Fig. 4.6. At this point EIT vs time and Pressure vs time plots were created and a user was asked to manually locate the beginning and end of the ramp maneuver. These start-end time values were stored for later use. Using the time vector and linear interpolation an index value (a number between 1 and length of the signal) was found for the start, end, and peak of the ramp maneuver. This was done for both the EIT and pressure data. But since the pressure sampling frequency was much higher than that of EIT the length of the two vectors did not coincide. To resolve this issue down sampling was done by finding the locations in the time vectors where the EIT time and pressure time were closest. This modified down sampling technique was done since the ratio of the two sampling frequencies was not an integer. With the completion of the timing index vector and down sampling, the GREIT based reconstruction was done. This GREIT based reconstruction was a second and more sophisticated reconstruction for the impedance images which were used in the final algorithm. The settings for the GREIT reconstruction are exampled in Chapter 4.2. 
The inverse using the GREIT model was performed with the reference voltage being the average of the first five samples from the start of the ramp maneuver. The ROI was found by locating any group of pixels which were connected with a minimum size of 20 pixels and were greater than $25 \%$ of the max EIT value [Pulletz et al., 2006].



Figure 4.6: Pressure and EIT data aligned so peak is at time 0 demonstrating the relationship between the pressure ramp the measured impedance difference. In this image the impedance difference is for the entire lung. 


\section{Chapter 5}

\section{Fuzzy Logic}

This chapter introduces the theory of Fuzzy Logic Systems (FLS). A FLS is a sequence of functions and methods which non-linearly map data (features) to a scalar output. It does this by turning crisp values (input data) into fuzzy sets (via fuzzification) and then back into crisp values (via defuzzification). A FLS is made up of three main components. 1) The fuzzifier takes the crisp input values(linguistic variables, which for this thesis are pressure and impedance change) and maps them into fuzzy sets with a degree of membership valued from 0 to 1 . From here the fuzzy sets are passed to the inference system which is composed of IF-THEN rules. These rules combine the fuzzy sets by using "AND" or "OR" operators resulting in another set of fuzzy sets called consequent sets, also with an according membership value. Finally the consequent sets are passed to the defuzzifier which combines the consequent sets and calculates a crisp value from the resulting curve. Each block can be seen in Fig. 5.1 with the accompanying decisions which need to be made for each block.

Fuzzy Logic is an excellent way to integrate engineering with expert knowledge. For this thesis it was a method to mix lung physiology and mechanical ventilation with medical imaging. In this thesis Fuzzy Logic was used along with Electrical Impedance 
Tomography to classify lung tissue as collapsed, normal, or overdistended. With this classification a Positive-end Expiratory Pressure (PEEP) was selected to maximize normal regions while minimizing collapsed and overdistended regions. The linguistic variables for this thesis were pressure and impedance change. With the fuzzy sets being Below, In Between, and Above. Finally the consequent sets for this thesis were: Collapsed, Normal, and Overdistended.



Figure 5.1: Fuzzy Logic Design Schematic. Adapted from [Mendel, 2001] 


\section{$5.1 \quad$ Fuzzifier}

\subsubsection{Fuzzy Sets}

Unlike classical sets where the distinction between member and non-member is clear, $\{0,1\}$, fuzzy sets can have a range of membership, $[0,1]$. The membership value dictates the degree to which they are associated to the fuzzy set, with higher values having larger associations. A fuzzy set can have elements which have varying degrees of membership, unlike classical sets where elements are either associated or not. Since membership is not complete a single element can have associations with multiple fuzzy sets. For instance an element $(x)$ with a range of possible values as dictated by universe $\mathbf{X}$ can have memberships to fuzzy sets $F_{1}$ and $F_{2}$. The membership for fuzzy set $F_{1}$ is indicated as $\mu_{F_{1}}(x)=\Re$ where $\mu_{F_{1}}(x) \in[0,1]$, similar with $F_{2}$ but with different subscripts. The association is performed by a membership function which takes elements, $x$, and places them in a fuzzy set. Fig. 5.2 displays an example of a membership function for input variable $x$ and fuzzy set $F_{1}$. It should be noted since elements can be associated with multiple fuzzy sets each fuzzy set has its own membership function.

Fuzzy sets also work with classical logical operations such as those displayed in Table 5.1. Within Table 5.1 operators which have no implementation can be broken down into union, intersection, and complement thus are not needed. With respect to the Law of Excluded Middles and the Law of Contradictions fuzzy logic is incongruent with classical logic. This is due to the inherent property of fuzzy sets and how they overlap as do their complements, thus you may never have complete membership or zero membership depending on which law is applied.

Multiple methods for performing the union and intersection are available and are referred to as t-conorms (union) and t-norms (intersection). A common alternative 


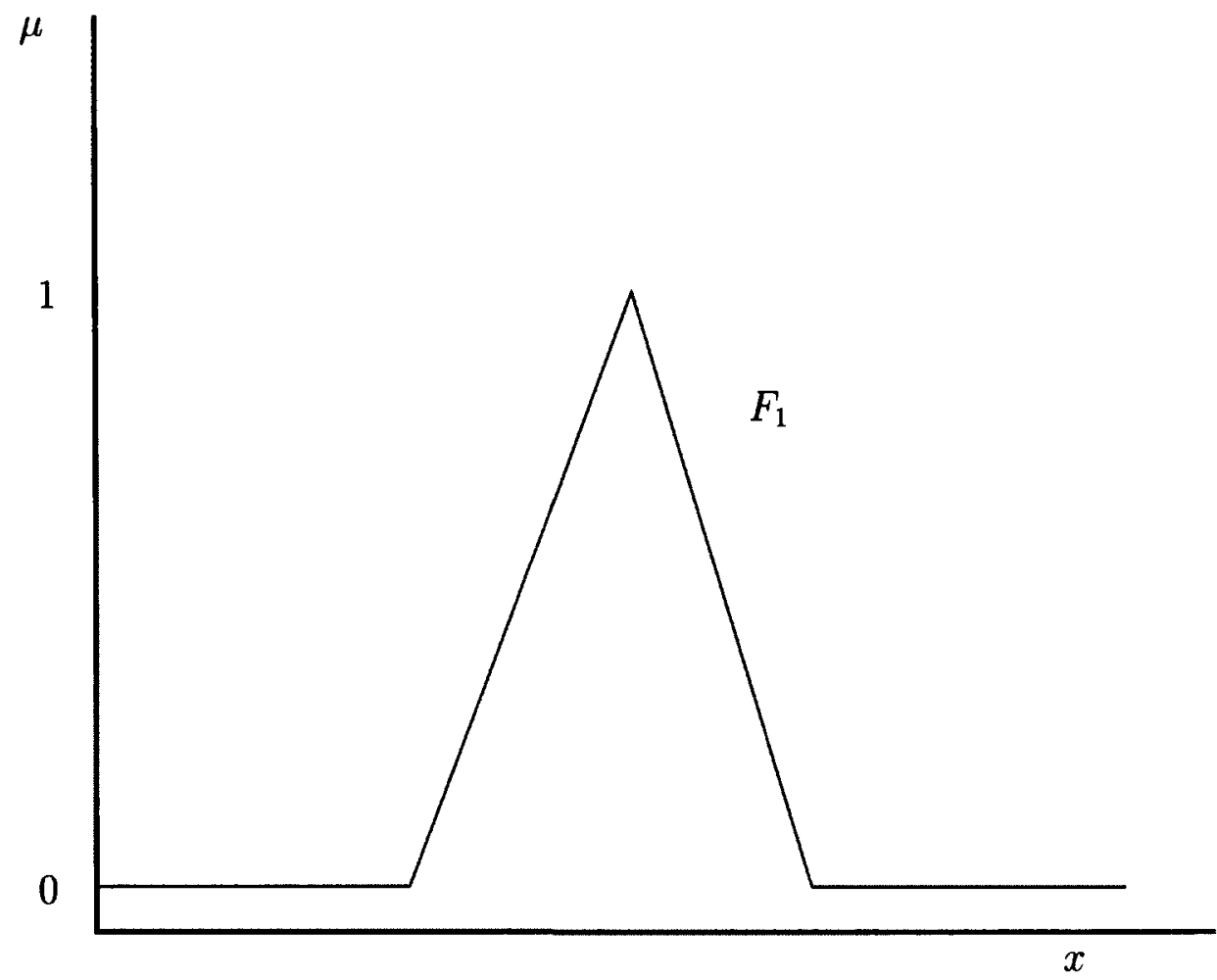

Figure 5.2: Triangle based membership function. Other types exist with this thesis using the trapezoidal function.

\begin{tabular}{|l|c|r|}
\hline Set Operator & Mathematical Format & Implementation Format \\
\hline Union & $\mu_{F_{1} \cup F_{2}}(x)=\mu_{F_{1}}(x) \vee \mu_{F_{2}}(x)$ & $\max \left(\mu_{F_{1}}(x), \mu_{F_{2}}(x)\right)$ \\
Intersect & $\mu_{F_{1} \cap F_{2}}(x)=\mu_{F_{1}}(x) \wedge \mu_{F_{2}}(x)$ & $\min \left(\mu_{F_{1}}(x), \mu_{F_{2}}(x)\right)$ \\
Compliment & $\mu_{F_{1}}(x)=1-\mu_{F_{1}}(x)$ & $1-\left(\mu_{F_{1}}(x)\right)$ \\
De Morgan & $\overline{F_{1} \cap F_{2}}=\overline{F_{1}} \cup \overline{F_{2}}$ & \\
Excluded Middle & $\overline{F_{1} \cup F_{2}}=\overline{F_{1}} \cap \overline{F_{2}}$ & \\
Contradictions & $F_{1} \cup \overline{F_{2}} \neq \mathbb{X}$ & \\
\hline
\end{tabular}

Table 5.1: Fuzzy Operators

to the $\max ()$ union operator is the algebraic sum operator and is illustrated in (5.1). A common alternative to the $\min ()$ intersection operator is the algebraic product operator and is illustrated in (5.2) [Mendel, 2001]. 


$$
\begin{gathered}
\mu_{F_{1} \cup F_{2}}(x)=\mu_{F_{1}}(x)+\mu_{F_{2}}(x)-\mu_{F_{1}}(x) \mu_{F_{2}}(x) \\
\mu_{F_{1} \cap F_{2}}(x)=\mu_{F_{1}}(x) \mu_{F_{2}}(x)
\end{gathered}
$$

\subsubsection{Membership Functions}

In a rule based FLS membership functions are used to take crisp values to fuzzy sets and vice versa. Theses fuzzy sets are then used in the antecedents and conclusions, refer to Fig. 5.1 to see the input of membership graphs to the two according fuzzy logic blocks. The main sections of any membership function are the Core, Support, and Boundary. The core region is the interval where the fuzzy set, for instance $F_{1}$, has full membership i.e. $\mu_{F_{1}}(x)=1$. The support region is the interval where membership is non-zero i.e. $\mu_{F_{1}}(x)>0$. The boundary region is the interval where the membership is between 0 and 1 i.e. $0<\mu_{F_{1}}(x)<1$. When designing fuzzy membership functions it is important to consider carefully the location of these regions as they play a large role in the performance of a FLS [Sivanandam et al., 2006].

Two types of membership functions exist. One which considers uncertainty in the data (Type-2) and the other which does not (Type-1). This thesis focuses on Type1 systems with extra detail on Type-2 systems available in [Mendel, 2001]. Within Type-1 systems two different fuzzy membership functions exist, singleton and nonsingleton. The difference between the two is an extra module, which models the datas uncertainties prior to fuzzification, for a non-singleton systems.

Referring to Fig. 5.1 for a overview of the variable names. For a singleton system $\mu_{\mathbf{X}}(\mathbf{x})=1$ if $\mathbf{x}=\mathbf{x}^{\prime}$ and $\mu_{\mathbf{X}}(\mathbf{x})=0$ for all $\mathbf{x} \neq \mathbf{x}^{\prime}$. Where $\mathbf{x}$ is equal to $p$ crisp elements each with there own universe. i.e. $\mathbf{x}=\left(x_{1}, \ldots, x_{p}\right)^{T} \in X_{1} \times X_{2} \times \cdots \times X_{p} \equiv \mathbf{X}$. 
$\mathbf{x}^{\prime}$ is the crisp input value of interest. From here the firing level is calculated by $\sup _{x_{i} \in X_{i}} \mu_{Q_{i}^{l}}\left(x_{i}\right) \equiv \mu_{X_{i}}\left(x_{i}\right) \star \mu_{F_{i}^{l}}\left(x_{i}\right)$, where $\star$ is a t-norm. For a singleton system the firing level is simplified since $\mu_{X_{i}}\left(x_{i}\right)$ is non-zero at one location, $x_{i}=x_{i}^{\prime}$. This calculated value is now the membership value for the fuzzy set $i$ and for rule $l$.

Setting up fuzzy membership functions can be done in multiple way, such as; intuition and neural networks [Mendel, 2001].

\subsection{Inference}

Fuzzy inferences are IF-THEN based rules from which consequences are derived from. Connectors between each term in the IF-THEN statement are made up of "AND" or "OR" which in turn have various mathematical equivalents, t-norms and t-conorms, respectively. The standard format for most fuzzy rules is IF (linguistic variable 1 is fuzzy set 1) AND/OR (linguistic variable 2 is fuzzy set 2) AND/OR ... THEN (output $\mathrm{n}$ is consequent n) [Sivanandam et al., 2006]. For instance IF $x_{1}$ is $F_{1}^{l}$ AND/OR ... AND/OR $x_{p}$ is $F_{p}^{l}$ THEN $y$ is $G^{l} . \quad l=1 \ldots m$ and represents the number of rules, $p$ represents the number of linguistic variables each with their own universe $\left(x_{1} \in X_{1} \ldots x_{p} \in X_{p}\right)$ and $y \in Y$ output linguistic variable for fuzzy set $G^{l}$. It can be shown that fuzzy systems with multiple outputs can be broken into multiple systems each with one output [Mendel, 2001].

\subsection{Defuzzifier}

Defuzzification is the processes of turning fuzzy sets into crisp values. Many methods of defuzzification exist with a main criteria being computational simplicity. The common ones are: Centroid, Center-of-Sums, Height, and Center-of-Sets [Mendel, 2001]. 


\subsubsection{Centroid Method}

The centroid method combines the consequent sets by using the max t-norm. $B=$ $\cup_{l=1}^{m} B^{l}$.

$$
y_{c}(\mathbf{X})=\frac{\sum_{i=1}^{N} y_{i} \mu_{B}\left(y_{i}\right)}{\sum_{i=1}^{N} \mu_{B}\left(y_{i}\right)}
$$

Where $i$ indexes the combined set $B$. This method is usually difficult to compute due to the union of the output fuzzy sets, $B^{l}$ [Mendel, 2001].

\subsubsection{Center-of-Sums}

This method combines the consequent sets by addition. $\mu_{B}(y)=\sum_{l=1}^{m} \mu_{B}^{l}(y)$. Then proceeds to find the centroid of $\mu_{B}(y)$.

$$
y_{a}(\mathbf{X})=\frac{\sum_{l=1}^{m} c_{B^{l}} a_{B^{l}}}{\sum_{l=1}^{m} a_{B^{l}}}
$$

Where $c_{B^{l}}$ is the centroid of the consequent set $B^{l}$ for rule $l$ and $a_{B^{l}}$ is the area underneath the consequent set $B^{l}$ for rule $l$.

\subsubsection{Height Defuzzifier}

This method locates the maximum point from the output set via a singleton method. For multiple maximums the average can be taken. It takes these values and adds then into a set of size $\mathrm{m}$, and then calculates the centroid of the resulting set.

$$
y_{h}(\mathbf{X})=\frac{\sum_{l=1}^{m} \bar{y}^{l} \mu_{B^{l}}\left(\bar{y}^{l}\right)}{\sum_{l=1}^{m} \mu_{B^{l}}\left(\bar{y}^{l}\right)}
$$

Where $\bar{y}^{l}$ is the location of the maximum value with $\mu_{B^{\prime}} \bar{y}^{l}$ being the according output set membership value. A common modifier to this method is to scale (5.5) by 
some measure of the spread of $\mu_{B^{l}}\left(y^{l}\right)$

$$
y_{m h}(\mathbf{X})=\frac{\sum_{l=1}^{m} \bar{y}^{l} \mu_{B^{l}}\left(\bar{y}^{l}\right) / \delta^{l^{2}}}{\sum_{l=1}^{m} \mu_{B^{l}}\left(\bar{y}^{l}\right) / \delta^{l^{2}}}
$$

\subsubsection{Center-of-Sets}

This method is fairly quick as it replaces the rule consequent set with a singleton located at the centroid with an amplitude scaled by the firing level, then finds the centroid of the combined singletons. The firing level $\left(f_{l}\right)$ is the combined membership value for each fuzzy set or $T_{i=1}^{p} \mu_{F_{i}^{l}}\left(x_{i}\right)$, where $T$ is short for the t-norm.

$$
y_{\cos }(\mathbf{X})=\frac{\sum_{l=1}^{m} c_{g^{l}} f_{l}}{f_{l}}
$$




\section{Part II}

\section{Contributions}




\section{Chapter 6}

\section{Inflection Points}

This chapter describes what inflection points (IP) are, how they are found, and their use with EIT in base pressure suggestion for mechanical ventilation systems. In this context inflection points are the locations along a pressure-volume/impedance (PV/PI) curve where the slope dramatically changes usually from a low compliance region to a high compliance region or a high compliance region to a low one. There exists multiple ways of locating IP [Harris et al., 2000] with this thesis exploring the sigmoid method, visual heuristics, and the 3-piece linear spline method. The basis behind this thesis is to locate pressure(s) where the alveoli are not in a state of collapse or overdistension which are known to be large contributors to ventilator induced lung injury (VILI) [Bigatello et al., 1999, Amato et al., 1998, Borges et al., 2006, Venegas et al., 1998, Takeuchi et al., 2002]. The use of inflection points in clinical settings are well known and are currently used to set the base-pressure (PEEP) in Acute Lung Injury (ALI) patients [Venegas et al., 1998, Harris et al., 2000]. In order to find inflection points which are meaningful particular ventilation strategies are used in order to reduce certain physiological effects and are described in detail in Chapter 2. 


\subsection{What are Inflection Points}

Inflection points are locations on a pressure-volume/impedance curve where alveoli efficiency increases/decreases dramatically depending on if the pressure is low or high respectively. There are various ways to locate the lower inflection point (LIP). The location where the pressure-volume curve starts to have a linear compliance [Matamis et al., 1984a, Brunet et al., 1995]. The pressure at which rapid increase in the compliance of the pressure-volume curve occurs [Harris et al., 2000]. Thirdly, to place two lines one along the low compliance region and the other on the high compliance region and locate the intersection [Takeuchi et al., 2002, Gattinoni et al., 1987, Amato et al., 1995]. Finally find the lower point in which the PV/PI curve first deviates from its high compliance region [Dambrosio et al., 1997]. All of these methods provide a distinct indication of a beginning and end of the high compliance regions.

\subsection{Importance of Inflection Points}

The work on inflection points is important as it is one of the common methods for optimizing PEEP in mechanical ventilation [Matamis et al., 1984b, Amato et al., 1998]. Patients in need of mechanical ventilation in particular patients with acute respiratory failure have a high mortality and morbidity rate [Hudson, 1989]. Mechanical ventilation can damage the lungs [Amato et al., 1998] causing lesions at the alveolar-capillary interface [Fu et al., 1992], create alterations in permeability [Carlton et al., 1990], and cause edema [Tsuno et al., 1990, Dreyfuss and Saumon, 1993]. VILI is a significant problem when it comes to critical patients with acute respiratory failure [Slutsky, 1994]. A contributing problem to VILI is the cyclic opening and closing of the alveoli [Ranieri et al., 1999, Pulletz et al., 2011, Mead et al., 1970] and can increase mortal- 
ity. [Amato et al., 1998] showed that two groups (one using protective ventilation strategies and one without) revealed higher survival rates in the protective ventilation group. From the experiment $11 / 29$ patients died in the protective ventilation group while 17/24 died in the non-protective ventilation group. [Amato et al., 1998] study using human subjects revealed that the length of being on mechanical ventilation impacted the survival after a 28-day trial period. A great deal of controversy exists in finding the appropriate PEEP settings with over 9000 papers published in this topic and no standard conclusion [Rouby et al., 2002]. With the use of EIT in clinical settings regional information is now available which will help in locating an optimal PEEP.

Some clinics use the lower and upper inflection points for pressure settings [Venegas et al., 1998]. In the study conducted by [Brunet et al., 1995] it was shown that the use of PEEP was able to increase normal regions while being able to decrease non-aerated regions. A study conducted by [Dambrosio et al., 1997] showed that the use of PEEP resulted in large reductions of collapsed regions. Albeit the given benefits of PEEP, there is a lack of unification amongst researchers on how to locate valid PEEP readings [Rouby et al., 2002]. Pressure-Volume curves have been suggested for finding the optimal PEEP value by looking for points which maximize the recruitment [Gattinoni et al., 1984]. Most current PV curves study the use of global pressurevolume readings to create a curve which is used to find associated parameters. The benefits of using global LIP and UIP are visible [Amato et al., 1998, Hinz et al., 2006]. [Hinz et al., 2006, Harris, 2005, Venegas et al., 1998] have suggested the use of UIP to set as the maximum airway pressure while [Lu et al., 1999, Takeuchi et al., 2001, Takeuchi et al., 2002, Harris et al., 2000, Venegas et al., 1998] have suggested using the LIP for the PEEP. It is also suggested that the PEEP should be set from the deflation limb since the alveoli would be recruited once and tend to collapse at a lower 
pressure once opened. This idea helps since PEEP is used to maintain open alveoli not to open them up initially [Papadakis and Lachmann, 2007, Albaiceta et al., 2004, Takeuchi et al., 2002, Harris et al., 2000].

[Papadakis and Lachmann, 2007] showed that when PEEP was set below the global LIP damage and collapse occurred most. In the same study by [Papadakis and Lachmann, 2007] it was also noticed that an increase in recruitment occurred, up to $40 \%$ of the lung, with a pressure set above the lower inflection point. [Muscedere et al., 1994] showed similar results to [Papadakis and Lachmann, 2007] in which large amounts of lung tissue were collapsed when PEEP was set to zero and below the lower inflection point. Much of the air volume was distributed to the anterior segments causing overdistension. This phenomenon was likely observed due to the use of animal subject in supine positions during lavage. When [Muscedere et al., 1994] set PEEP above the LIP collapse occurred in the early stages and progressively inflated over 2 hours with large areas expanding first followed by smaller focal areas. The total airway injury score for the PEEP > LIP group was similar to that of the control group. A similar independent study was conducted by [Takeuchi et al., 2002] and found setting the PEEP to LIP +2 mbar provided the best compliance and best arterial oxygen partial pressure at a fraction of inspired oxygen of 0.5 . LIP $+2 \mathrm{mbar}$ also minimized lung inflammation and mRNA expression for interleukin - $1 \beta$, which along with mRNA expression for interleukin- 8 are inflammatory responses indicative of inadequate PEEP [Takeuchi et al., 2002]. [Amato et al., 1995] conducted an experiment displaying the benefits of using LIP for PEEP. [Amato et al., 1995] set the PEEP above the LIP with tidal volume $<6 \mathrm{ml} / \mathrm{kg}$ and peak pressure $<40 \mathrm{mbar}$ and compared it to a volume cycled ventilation with $V_{t}<12 \mathrm{ml} / \mathrm{kg}$, PEEP set via $\mathrm{FIO}_{2}$, and normal $\mathrm{PaCO}_{2}$ levels. The experiment found that the first scheme improved oxygen-blood transaction in patients with ARDS increasing the chance of 
early weaning and lung recovery. The first scheme was also better in $\frac{\mathrm{PaO}_{2}}{\mathrm{FIO}_{2}}$, compliance, and higher weaning rates, but had no significant improved survival rate. The survival rates were $5 / 15$ and $7 / 13$ for first and second scheme respectively. It is clear that the use of PEEP has its benefits and setting PEEP to the LIP or slightly above works quite well.

In the past global PV curves were one of the best available methods for PEEP selection but with recent advancements, in particular EIT, regional information is now attainable [Kunst et al., 2000, Meier et al., 2008, Hinz et al., 2006, Pulletz et al., 2011, Frerichs et al., 2003, Hinz et al., 2003b, Victorino et al., 2004, Grychtol et al., 2009, Wolf et al., 2010]. Due to the heterogeneity of air distribution within diseased lung tissue recruitment happens along the entire PV curve. With regional information being available this phenomenon can be better understood [Hinz et al., 2006, Pulletz et al., 2011] and hopefully more accurate PEEP selections can be found.

\subsection{Techniques to Locate Inflection Points}

To locate inflection points multiple methods exist with this thesis testing three methods. The first method discussed below is visual heuristics. Many clinics already use this method to locate inflection points. The second and more classic automated approach is the sigmoid model (6.3.2). This is an automated method which fits the PV/PI data to a sigmoid function using a particular minimization criteria and regression algorithm. [Venegas et al., 1998] and [Grychtol et al., 2009] used the LevenbergMarquardt regression algorithm and minimized the sums of squared residual, in this thesis the trust region reflective algorithm was used with the sums of squared residual minimization criteria. When compared the trust region reflective algorithm found the 
same optimal parameters as the Levenberg-Marquardt algorithm. The last and fairly new approach is to use a three-piece linear spline (6.3.3). [Grychtol et al., 2009] used a least squares minimization in their study with this thesis using a partitioned least squares criteria. The function used in this thesis was created by [D'Errico, 2011] and is explained in Chapter 7.

\subsubsection{Visual Heuristics}

In the clinical setting inflection points are located by the clinician using their expert knowledge from a global pressure-volume curve. These inflection points are then used as the PEEP value with lower inflection point +2 mbar being a common choice [Harris et al., 2000, Matamis et al., 1984a, Venegas et al., 1998, Martin-Lefevre et al., 2001, Takeuchi et al., 2002]. Variability between visual based IP exists within clinical settings [Harris et al., 2000]. When compared to the sigmoid method [Venegas et al., 1998] visual inspection produced a 0.89 and 0.94 correlation between static and quasi static curves [Martin-Lefevre et al., 2001]. [Martin-Lefevre et al., 2001] also compared the visually inspected inflection points to a linear segmental regression method and found a correlation of 0.54 and 0.84 for the lower and upper inflection points respectively. [Rossi et al., 2008] conducted a study in which the LIP were found by multiple people and averaged together with a repeat of the experiment if the two inflection points differed by more then 2 mbar. In this study the lower inflection point were reported to be similar to other papers using the same animal model and showed that setting PEEP to the lower inflection point achieves more normal states compared to High Frequency Oscillatory Ventilation (HFOV) when the possible states were collapsed, normal and overdistended [Rossi et al., 2008] 


\subsubsection{Sigmoid Function}

[Venegas et al., 1998] introduced the use of the sigmoid model as an automated method to find inflection points and has been used by many other researches since [Hinz et al., 2006, Grychtol et al., 2009, Albaiceta et al., 2004, Harris et al., 2000]. With the usage of the sigmoid function in global pressure-volume curves clinicians were able to locate both upper and lower inflection points to help reduce cases of cyclic opening and closing and overdistension. The idea of using a sigmoid function was to obtain a method which would allow for non zero asymptotic limits [Salazar and Knowles, 1964, Colebatch et al., 1979].

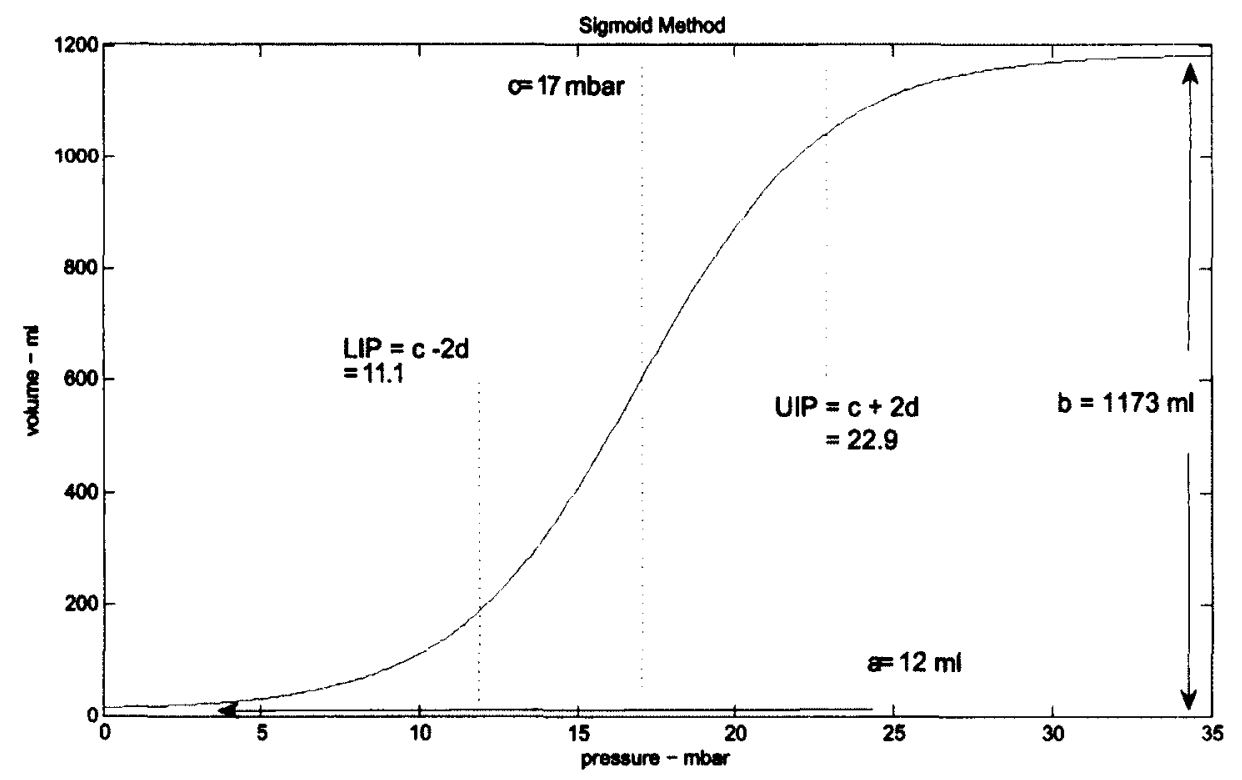

Figure 6.1: Sigmoid function fit illustrating the location of each parameter. The data in this model was the same data used for the entire experiment. Each parameter is labeled and displays its value at optimization. Parameter $d$ can be found using the value of ' $c$ ' the inflection points and (6.2)

The sigmoid method fits the data to (6.1) where $V$ is the inflation or absolute volume, $P$ is the airway or transpulmonary pressure, $a$ is the volume corresponding the lower asymptote which is used to approximate the residual volume when abso- 
lute volume is used, $b$ is the vital capacity or total volume change between the two asymptotes, $c$ is the pressure at the highest compliance and is also the true inflection point, and $d$ is proportional to the pressure range where most of the volume changes takes place, or in other words it's the pressure difference between the actual inflection and the zone of high compliance. The inflection points are located using (6.2) which are also the locations where the tangents from the two asymptotes intersect with the tangent at the best compliance $(P=c)$. Constraints on the final parameters were also set in order to achieve logical solutions. For parameters $b, c, d$ a lower bound of 0 was set while parameters $c, d$ had an upper bound of the maximum pressure. All other constraints were set to $-\infty$ and $\infty$. The initial search parameters used were $a=\min ($ of impedance), $b=\max$ (of impedance) - $\min ($ of impedance), $c=$ median (of pressure), $d=0.5$. An example of the the fitted sigmoid and the inflection points calculated can be seen in Figure 6.2. The data used in this thesis is explained in [Pulletz et al., 2011] and in Chapter 4.

$$
\begin{gathered}
V=a+\left[\frac{b}{1+e^{-(P-c) / d}}\right] \\
P_{u, l}=c \pm 2 d
\end{gathered}
$$

\section{Sigmoid method on EIT data}

With every stride forward new challenges appear and the use of the sigmoid method on global PV curves is no exception. In current mechanical ventilation systems the assumption that the lungs behave in a global homogeneous manner is false [Grychtol et al., 2009, Hinz et al., 2006]. [Hinz et al., 2006] showed with the use of an EIT system that the regional information of pressure-impedance curves could pro- 
vide some important insight into possible optimal pressure values.

\subsubsection{Three - Piece Linear Spline}

A main objective of this thesis was to test the three-piece linear spline for locating inflection points within EIT data. The three piece linear spline fits PV/PI curves with three linear segments with optimal break point placement. (6.3) shows the mathematical definition where $a$ indicates the respective slopes and $b$ the respective $\mathrm{y}$-intercept. This thesis implemented the partitioned least squares criteria and is explained in Chapter 7. The MATLAB implementation was designed and coded by John D'Errico [D'Errico, 2011]. [Grychtol et al., 2009] used a slightly different algorithm with additional constraints on the first and last line segment. [Grychtol et al., 2009] ensured the first and last segments had a specific range of slope, this idea is looked at in Chapter 9. In this thesis to ensure three distinct line segments were placed the length of each segment was set to be at least 1 mbar. The inflection points were the locations where each line segments meets its adjacent which is also the location of the break points. An example of a three-piece fitting can be seen in Figure 6.2. The linear spline method is preferred over the sigmoid method because of its resemblance to manual curve analysis[Hinz et al., 2006]. Despite the relative novelty of this method it has a great intuitive appeal as it picks out the location of the high compliance rather easily which has shown to resemble normal lung function [Harris, 2005].

$$
V / I= \begin{cases}a_{1} P+b_{1} & 0 \leq P \leq \mathrm{LI} \\ a_{2} P+b_{2} & \mathrm{LI} \leq P \leq \mathrm{UI} \\ a_{3} P+b_{3} & \mathrm{UI} \leq P\end{cases}
$$




\subsection{Results}

The results from the comparison between the sigmoid method and linear method, along with the comparison of the visual heuristic method with the linear method and sigmoid method can be seen in Chapter 8 .

\subsection{Summary and Discussion}

In summary currently some clinics are using inflection points to set the PEEP value for conventional mechanical ventilation [Harris et al., 2000]. An issue which was raised earlier is the variability in locating the inflection points, this can be seen as there exists various method in which to locate the lower inflection point. The first is to locate the pressure where the PV curve starts its trend of high compliance [Matamis et al., 1984b, Brunet et al., 1995]. The second is to locate the pressure where a rapid increase in slope occurs [Harris et al., 2000]. The third is to locate the pressure where the tangents of the lower compliance and high compliance sections cross [Takeuchi et al., 2002, Gattinoni et al., 1987, Amato et al., 1995]. The fourth method is to locate the lower pressure point where the curve first deviates from its high compliance region [Dambrosio et al., 1997]. Model based techniques were created to help curb this variability and to improve reproducibility. [Venegas et al., 1998] introduced the sigmoid method and [Grychtol et al., 2009] used the 3-piece linear method. Another point which should be noted in this study and others is that for all pressurevolume/impedance curves the pressure is assumed to be homogeneous throughout the lungs [Hinz et al., 2006]. In addition when using regional information from the EIT the image is restricted to one plane and does not consider the cephalo-caudal plane [Hinz et al., 2006]. 

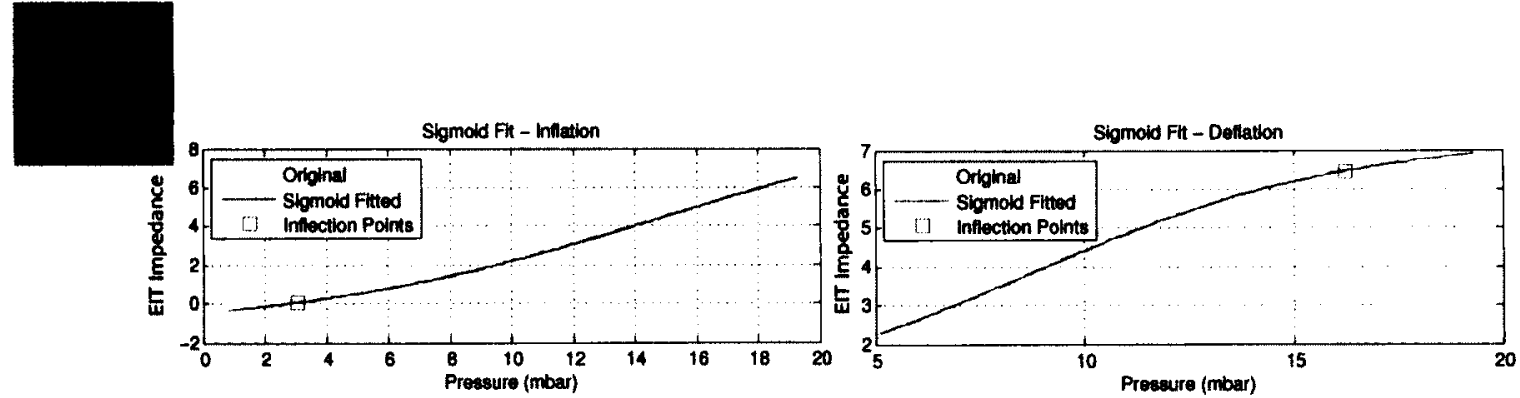

(a) Sigmoid Fitted - Patient 08
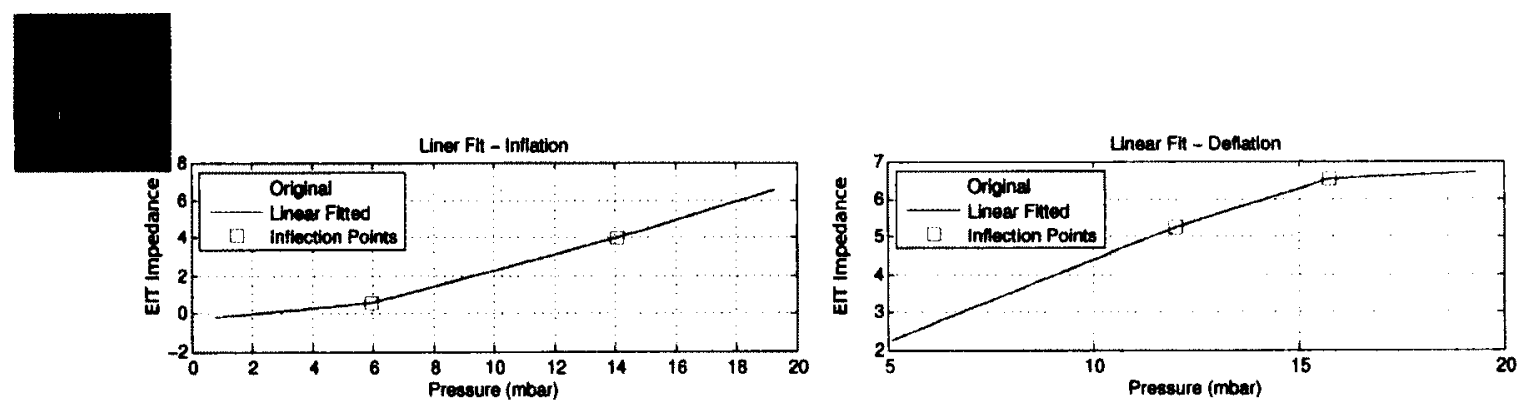

(b) Linear Fitted - Patient 08
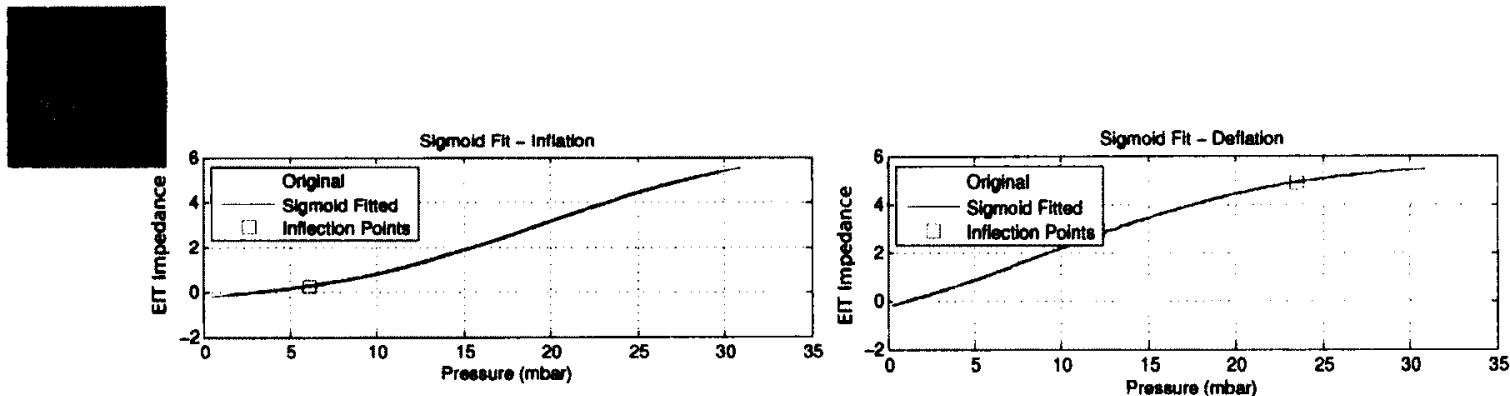

(c) Sigmoid Fitted - Patient 15
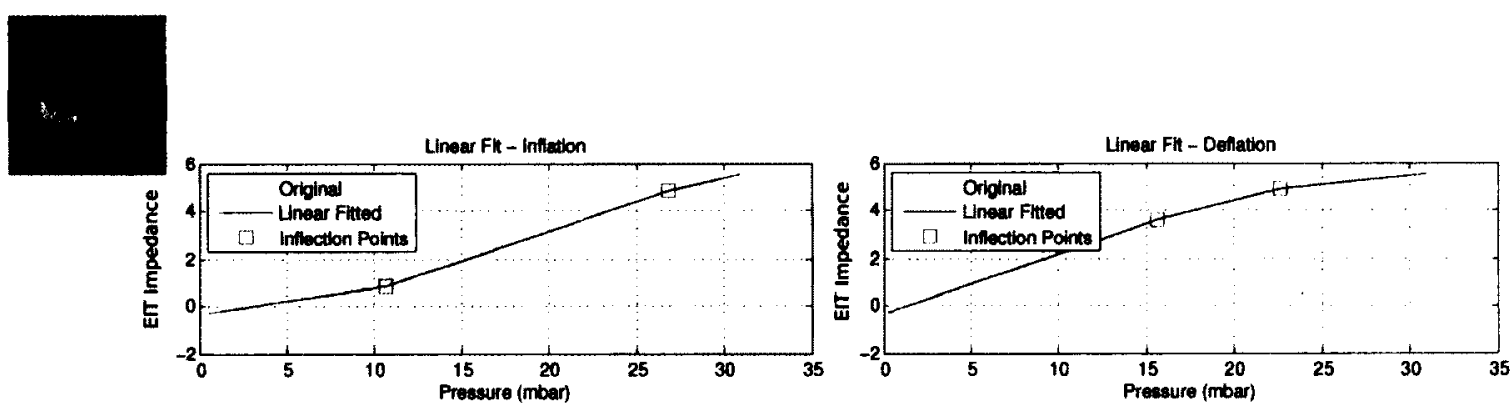

(d) Linear Fitted - Patient 15

Figure 6.2: Two examples of a sigmoid and linear spline fitted pressure-impedance curves with inflection points. The pressure-impedance data was selected from the indicated spot in the EIT lung image in the top left corner. It can be seen from the sigmoid graphs that most often both IP are not found, while the linear spline method is always able to locate an IP. 


\section{Chapter 7}

\section{Algorithm Design}

This chapter explains the optimal pressure algorithm designed for the use of taking EIT images and the associated pressure ramp and to calculate the optimal Positive End-Expiatory Pressure (PEEP) to reduce 'bad' (overdistended and collapsed) lung tissue while maximizing 'good' (well ventilated) lung tissue. The algorithm was designed and coded in the MATLAB language and was designed to integrate into preexisting data analysis code from [Pulletz et al., 2011]. The system first calculates the Inflection Points (IP) from the Pressure-Impedance (PI) graphs, then applies the IP along with expert knowledge of guided ventilation strategies to create fuzzy membership graphs for a Fuzzy Logic System (FLS), and finally chooses a PEEP. Two Fuzzy Logic systems were created for comparison, with one using the pressure values $\left(F S_{p}\right)$ of the IP while the other used the EIT values $\left(F S_{e}\right)$.

Using the designed algorithm optimal PEEP settings were found on the inflation and deflation limbs. With multiple patients in the data this chapter will display examples, results, and overall stats of specific patients or for the entire population. Using IP as the main source for the membership graphs allowed for past literature (Chapter 6) to be used in locating an optimal PEEP. The benefits of the approach here 
is that improvements can be done in parallel. I.E. improving the IP location algorithm and improvements to the FLS can be done at the same time. [Grychtol et al., 2010] worked on a similar task but did not use the information provided by the IP and had more complicated rules in his system. Along with the parallel nature of the design the ventilation recruitment maneuver was under 2 minutes in length. The last novel aspect is how regional IP were used when locating the PEEP.

The algorithm is designed into four stages. The first being the locating of the inflection points. The second is the fuzzification. The third is the premise calculation and fourth is defuzzification and optimization (Fig. 7.1). 


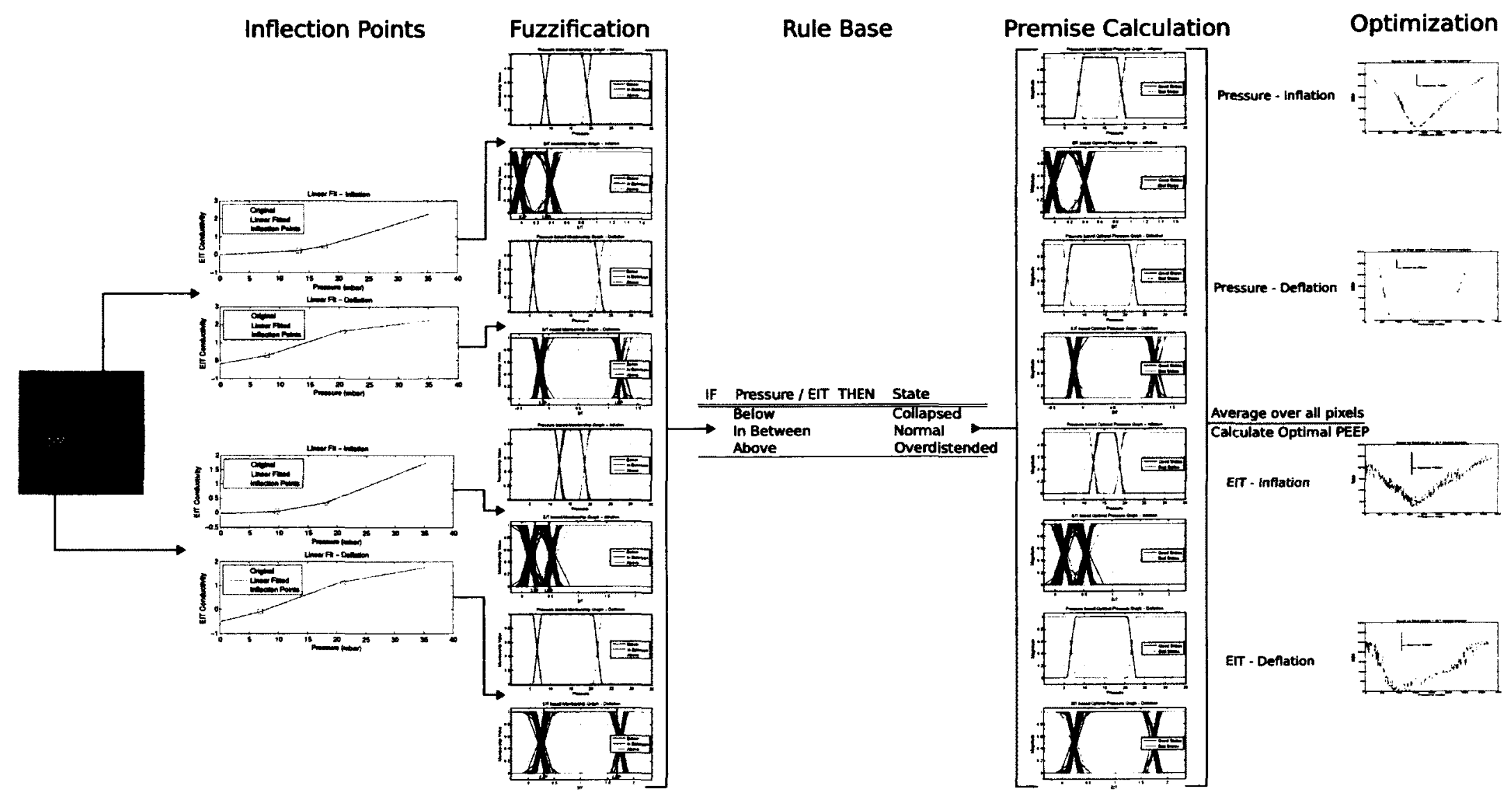

Figure 7.1: Example outputs from each stage of the algorithm. Starting with inflection point location the algorithm works toward fuzzification using the IP found the previous stage for the membership graphs. Once applying the rule base the premise is created and shown clearly. Finally each pixel is average and the PEEP is selected as shown in the last column. 


\subsection{Inflection Point Calculation}

To create the Fuzzy Logic system, regional inflection points were used within the membership graphs. In order to calculate the inflection points the pressure ramp and EIT images were first split into two sections: a) inflation limb and b) deflation limb. Details on the pre-analysis are discussed in Chapter 4.2.3. From here each pixel within the ROI was fitted to a three-piece linear spline in the partitioned least squares sense and the IP located. Fig. 7.1 shows an example of the expected output.

The three-piece linear spline algorithm computes the piecewise linear least squares spline with optimized internal breakpoints. It uses a partitioned least squares for the parameter estimation and was coded by [D'Errico, 2011]. Initial breakpoint values used for the linear fit were equally spaced value ranging from the lowest to highest pressure. From here the matrix $A$ and $b$ were set to solve the inequalities in (7.1). In (7.1) all inequalities, except $A x \leq b$ were not used. The three constraints which made up $A$ and $b$ are listed below. Where $X_{i n}$ is the input data on the x-axis. $a$ is the second break point, $b$ is the third break point, with the first and fourth break point being the min and $\max$ of the $X_{i n}$ data and $l_{\min }$ is the minimum length of each line segment.

1. $a>l_{\min }+\min \left(X_{i n}\right)$

2. $b-a>l_{\min }$

3. $b \leq \max \left(X_{i n}\right)-l_{\min }$

(7.1) represents the constraints for the minimization where $c(x)$ and $c_{e q}(x)$ are non-linear functions which output a vector. $x$ is a vector of the unknown parameters. $A$ and $A_{e q}$ are a set of linear equations with inequalities/equalities in $b$ and $b_{e q}$. Finally 
$l_{b}$ and $u_{b}$ are the lower and upper bounds for the parameters, $x$. In this thesis $x$ was the two break points.

$$
\min _{x} f(x) \text { such that } \begin{cases}c(x) & \leq 0, \\ c_{e q}(x) & =0, \\ A \cdot x & \leq b \\ A_{e q} \cdot x & =b_{e q} \\ & l_{b} \leq x \leq u_{b}\end{cases}
$$

\subsection{Fuzzification}

In order to create a FLS, membership functions are needed to map the crisp values to corresponding fuzzy sets (Fig. 7.1). Using trapezoidal membership graphs three fuzzy sets were created: Below, In Between, and Above. The states were created as such because of the known benefits of setting PEEP above the lower inflection point and below the upper inflection point. Studies showing the relationship between having PEEP below the LIP and above the UIP and the amount of lungs collapsed or overdistended are discussed in detail in Chapter 6. The first step in the process was to separate the inflation and deflation limb which was done via the inputed timing data. From here the next step was to set up the membership graphs. Using the trapezoidal method each membership graph takes 4 inputs. The first, (a), indicates where the upward slope starts with prior to reaching that point the membership is zero. The second, (b), indicates where the upward slope ends and the constant full membership line begins. The third, (c), indicates where the full membership ends and the decrease slope begins. The fourth, (d), parameter indicates where the slope stops and the membership graph goes to zero (Fig. 7.2). Each pixel in the 
ROI had a slightly different shaped membership graph due to the location of their respective IP (Fig. 7.1). For a detailed look of how the parameters are set for the membership graphs refer to Table 7.1. Table 7.1b has the constant $f$, this constant is used to convert pressure (mbar) to image amplitude $(\Delta Z)$. This was done using the calibration equation (7.2). In this equation $p_{v e c}$ is the pressure vector, $\Delta Z_{v e c}$ is the image amplitude vector, $p$ is the known value of $\pm 2 \mathrm{mbar}$, and $\Delta Z$ is the unknown factor, $f$.

$$
\frac{\max \left(p_{v e c}\right)}{\max \left(\Delta Z_{v e c}\right)}=\frac{p}{\Delta Z}
$$

\begin{tabular}{|l|c|c|c|c|}
\hline Membership & Input 1 (mbar) & Input 2 (mbar) & Input 3 (mbar) & Input 4 (mbar) \\
\hline \hline Below & $\min (\mathrm{p})$ & $\min (\mathrm{p})$ & $-2+$ LIP & LIP \\
In Between & $-2+$ LIP & LIP & UIP & $2+$ UIP \\
Above & UIP & $2+$ UIP & $\max (\mathrm{p})$ & $\max (\mathrm{p})$ \\
\hline
\end{tabular}

(a) Linguistic variable pressure membership graph parameters

\begin{tabular}{|l|c|c|c|c|}
\hline Membership & Input 1 $(\Delta Z)$ & Input 2 $(\Delta Z)$ & Input 3 $(\Delta Z)$ & Input 4 $(\Delta Z)$ \\
\hline \hline Below & $\min (\mathrm{e})$ & $\min (\mathrm{e})$ & $-2 \mathrm{f}+\mathrm{LIP}$ & LIP \\
In Between & $-2 \mathrm{f}+$ LIP & LIP & UIP & $2 \mathrm{f}+$ UIP \\
Above & UIP & 2f+UIP & $\max (\mathrm{e})$ & $\max (\mathrm{e})$ \\
\hline
\end{tabular}

(b) Linguistic variable EIT membership graph parameters

Table 7.1: Details on creating the trapezoidal based fuzzy membership functions

To calculate the degree of membership for a trapezoidal membership graph one can either use the R2011a Fuzzy Logic Toolbox in MATLAB, if available or use Equation 7.3 which simplifies to an easy to implement equation: $f(x: a, b, c, d)=$ $\max \left(\min \left(\frac{x-a}{b-a}, 1, \frac{d-x}{d-c}\right), 0\right)$. The later method was implemented, giving control over software details. 


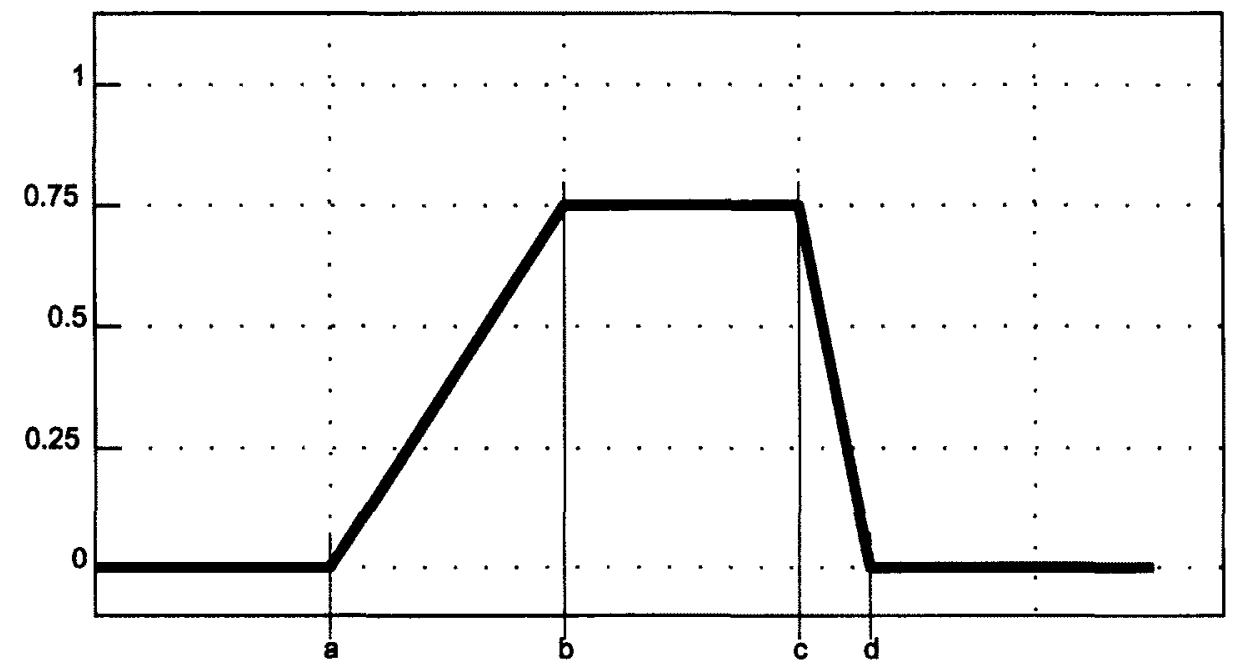

Figure 7.2: Trapezoidal Fuzzy Membership graph. This is the type of membership graph used within the designed algorithm.

$$
f(x: a, b, c, d)= \begin{cases}0, & x \leq a \\ \frac{x-a}{b-a}, & a \leq x \leq b \\ 1, & b \leq x \leq c \\ \frac{d-x}{d-c}, & c \leq x \leq d \\ 0, & d \leq x\end{cases}
$$

\subsection{Premise Calculation}

Once the two linguistic variables (pressure and impedance) were fuzzified they proceeded to the Inference (IF-THEN) system. For this thesis two rule sets were used, both were simple rules with one linguistic variable allowing for traceability. With minimal literature on this specific topic a simple basis in which to expand from was needed [Grychtol et al., 2010]. Using previous PEEP setting literature as a base three simple rules were derived and can be seen in the Rule Base Table 7.2. 
The similarities to past lung physiological papers exist. Looking at the first rule used if the pressure is found below the LIP it is considered collapsed which resembles many observations found in literature much of which is discussed in Chapter 6. This also applies to the overdistended state but with the key difference being if the pressure exceeds the UIP the lung unit is considered overdistended. [Grychtol et al., 2010] used more complex rules, as illustrated in (8.3) but for this thesis simplicity was the aim. The use of simple rules allowed for ease of testing the effects of the rules and provide a basis in which to develop on.

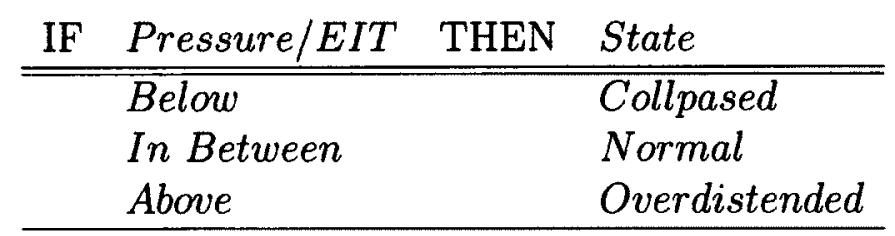

Table 7.2: Rule base table for the first and second fuzzy systems

\subsection{Defuzzification}

The last section of a FLS is to establish the defuzzification system. [Grychtol et al., 2010] used max defuzzification to decide which state the pixel would be end in. This entails taking the maximum of all consequents and choosing the fuzzy set with the max membership as the final class. This method would have been performed on each pixel at every time step. The MAX method is easy to implement but loses information by not considering the other states in the final classification. Other methods of defuzzification provide an adjustment based on the other consequent memberships, for instance finding the center of mass below the consequent curve. For a classification problem, like this thesis, more complicated defuzzification procedures can pose a problem since the final class must discrete. Defuzzification maps the consequents to a crisp out value, such as pressure. So for each input pressure to the FLS 
the output would also be a pressure. This would work fine for a control system but for our purpose, classification, we don't have a single input measurement but rather we are looking for an optimal PEEP from an entire range of pressures. For this thesis the MAX method was not used since the membership degree was kept to use all the information available in the pressure optimization.

The pressure optimization was done by categorizing each of the consequents into a 'good' and 'bad' category. The 'good' category consisted of units within the normal consequent state while the 'bad' category consisted of units within the collapsed and overdistended consequent states. From here the sum for all the pixels were taken with the logic that a single pressure must be selected for the entire ventilation system. This leads to working with two 1-D vectors with the $\mathrm{x}$-axis being the index for the optimal pressure. This is shown in Fig. 7.1 as the last section in the diagram, Optimization. To find the optimal index the bad states was subtracted from the good states and the global max was found of the resulting curve. For examples refer to Fig. 7.1.

\subsection{Summary and Discussion}

In summary this algorithm was designed to use the regional information from an EIT system to locate regional IP with the ultimate means of locating an optimal PEEP pressure. The steps taken were to first fit the PI graphs to a three-piece linear spline. From here the inflection points were taken as the location of the breakpoints where the pressure and impedance change values were used to create membership graph for two FLS. The membership graphs map the pressure and impedance change to 3 fuzzy sets: Below, In Between, and Above. The significance of these fuzzy sets are that they ensure the that optimal PEEP is found somewhere between the two inflection points. The significance of this point is discussed in Chapter 6. Once 
fuzzified the fuzzy sets are used in IF-THEN rules to create 3 conclusions: Collapsed, Normal, and Overdistended. The rules for each system can be seen in Table 7.2. With the conclusions now established for each pixel they are put into 'good' and 'bad' categorizes. The 'good' category consists of pixels within the Normal conclusion with the 'bad' category consisting of the Collapsed and Overdistended conclusions. From here the sum of the membership values for the two categories is done and the difference between 'good' and 'bad' is taken to find the maximum of the difference. The max location signifies the index for the optimal pressure. Finding the best PEEP is a matter of using the index in the pressure vector.

The system created here is a large first step into using both EIT and Fuzzy Logic for the purpose of PEEP adjustment. 


\section{Chapter 8}

\section{Results and Analysis}

This chapter explains the results from the algorithm described in Chapter 7. The data used for comparison and analysis is described in Chapter 4. This chapter goes through a comparative analysis of the linear and sigmoid methods, the linear and visual heuristic methods, the sigmoid and visual heuristic methods, analysis of the membership graphs, and finally an analysis of the PEEP selection.

\subsection{Inflection Points}

This section compares the inflection points (IP) using the linear method, the sigmoid method, and the visual heuristic method.

\subsubsection{Inflection Points for Individual Algorithms}

To find the regional IP a 3-piece linear spline [Grychtol et al., 2009] was used with optimal breakpoint location [D'Errico, 2011]. The algorithm is described in Chapter 7. When comparing LIP found between control and ALI patients, a noticeable difference in value was found with the ALI patients having an average LIP of 10.7146 
mbar versus an average LIP of 6.3550 mbar in control patients. This result matches [Pulletz et al., 2011] as they found similar increases, in the same data, using the regional opening technique.

\subsubsection{Linear Spline vs. Sigmoid Method}

To compare the linear and sigmoid methods locations where both IP were found could only be used. The linear method was able to find inflection points $99.95 \%$ of the time while the sigmoid method was only able to an inflection point $57.13 \%$ of the time. The sigmoid method had an interesting trend in that it tended to find one inflection point, either lower or upper but hardly ever both (Fig. 8.1).
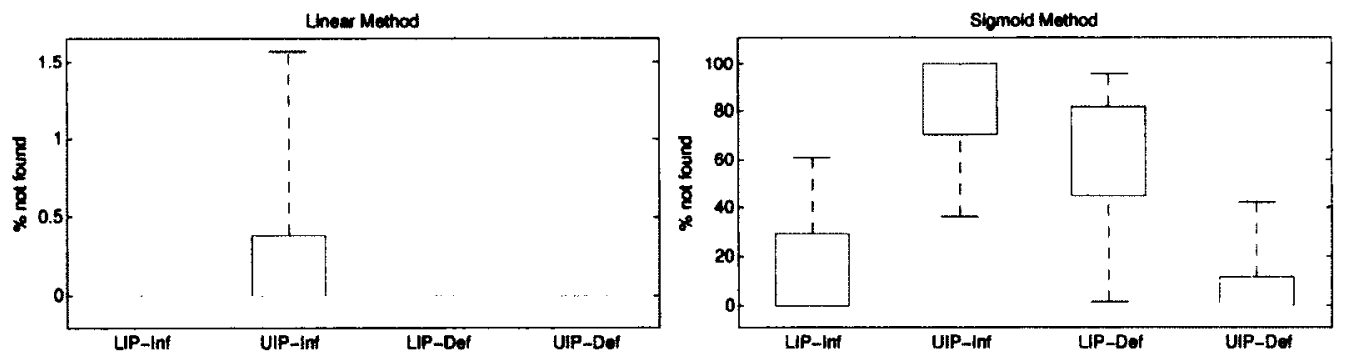

Figure 8.1: How frequent each curve fitting method was able to locate an IP. The box around the median line represents the $75^{\text {th }}$ to $25^{\text {th }}$ quartile. The dashed lines outside of the box indicates the max and min values.

Within Fig. 8.2 a single red line with no quartile box means only one difference was obtained. This is because one of the two methods was unable to locate an IP, thus no comparison could be taken. For some patients where no comparison was available because neither algorithm could find an IP in the same pixel and location a N/A was placed. When the sigmoid and linear methods were compared and averaged over the entire patient population the difference between the two methods for the lower inflection point on the inflation limb were similar, yielding a 1.47 mbar average difference with average standard deviation of 3.02 and average median difference of 
1.50 mbar. A full listing of the differences for both inflection points and both pressure arms can be seen in Table 8.1. From Fig. 8.2 we can see the difference between the two methods being small, but have large variability. When looking at the average absolute difference large differences of $4.23 \mathrm{mbar}$ was found. The value was calculated as the average absolute difference over both limbs and all IP. This value is significant considering that this may change the state in which the alveoli is in. From Table 8.1 it is clear the larger differences originated from the UIP on the inflation limb and the LIP on the deflation limb.

\begin{tabular}{|c|c|c|c|}
\hline & Mean & Std & Median \\
\hline \hline LIP - Inflation & 1.47 & 3.02 & 1.50 \\
UIP - Inflation & -6.80 & 2.54 & -6.82 \\
LIP - Deflation & 4.07 & 1.84 & 4.07 \\
UIP - Deflation & -2.37 & 2.24 & -2.78 \\
\hline
\end{tabular}

Table 8.1: Mean, standard deviation, and median difference between the sigmoid and linear inflection points averaged over all patients. The units are in mbar. For a graphical representation refer to Fig. 8.2.

One explanation for the large absolute difference is the amount of samples in each average. In the sigmoid method a median of $3.29 \%$ of all the pixels in patients could find an UIP on the inflation limb while $30.10 \%$ could find a LIP on the deflation limb. The lack of samples in the comparison can suggest that it does not give a correct representation of the actual data. Testing to see if the \% of IP found effects the magnitude of the difference (8.1) was used, where $d_{a}$ is the absolute difference between the two IP found by the linear and sigmoid methods and $p p$ is the percent of IP not found by the sigmoid method.

$$
r=\operatorname{corr}\left(d_{a}, p p\right)
$$

A correlation of $r_{\% \text { diff-uipinf }}=0.0360(p=0.8769)$ was found for the UIP on the 

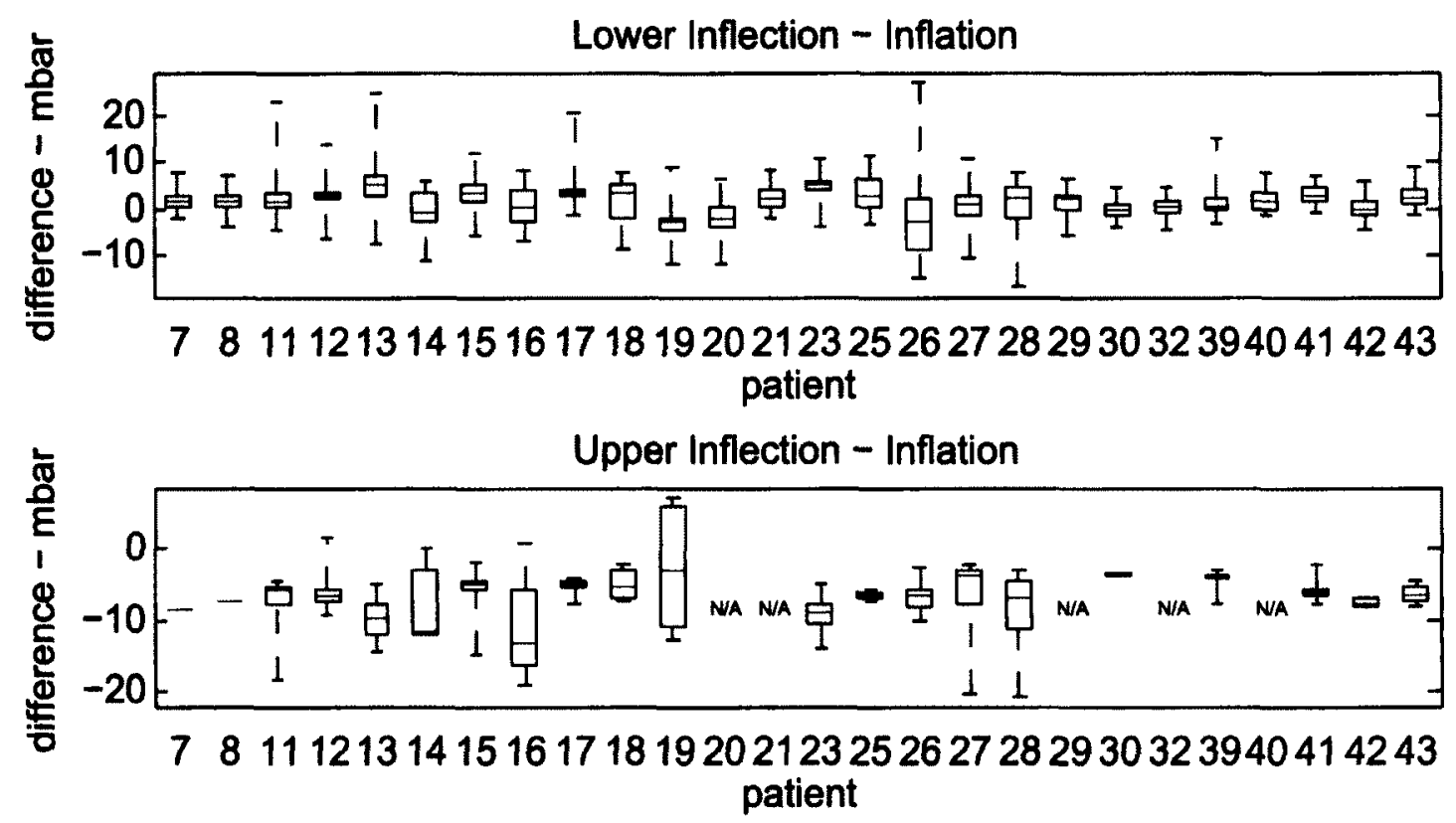

(a) Inflation Arm
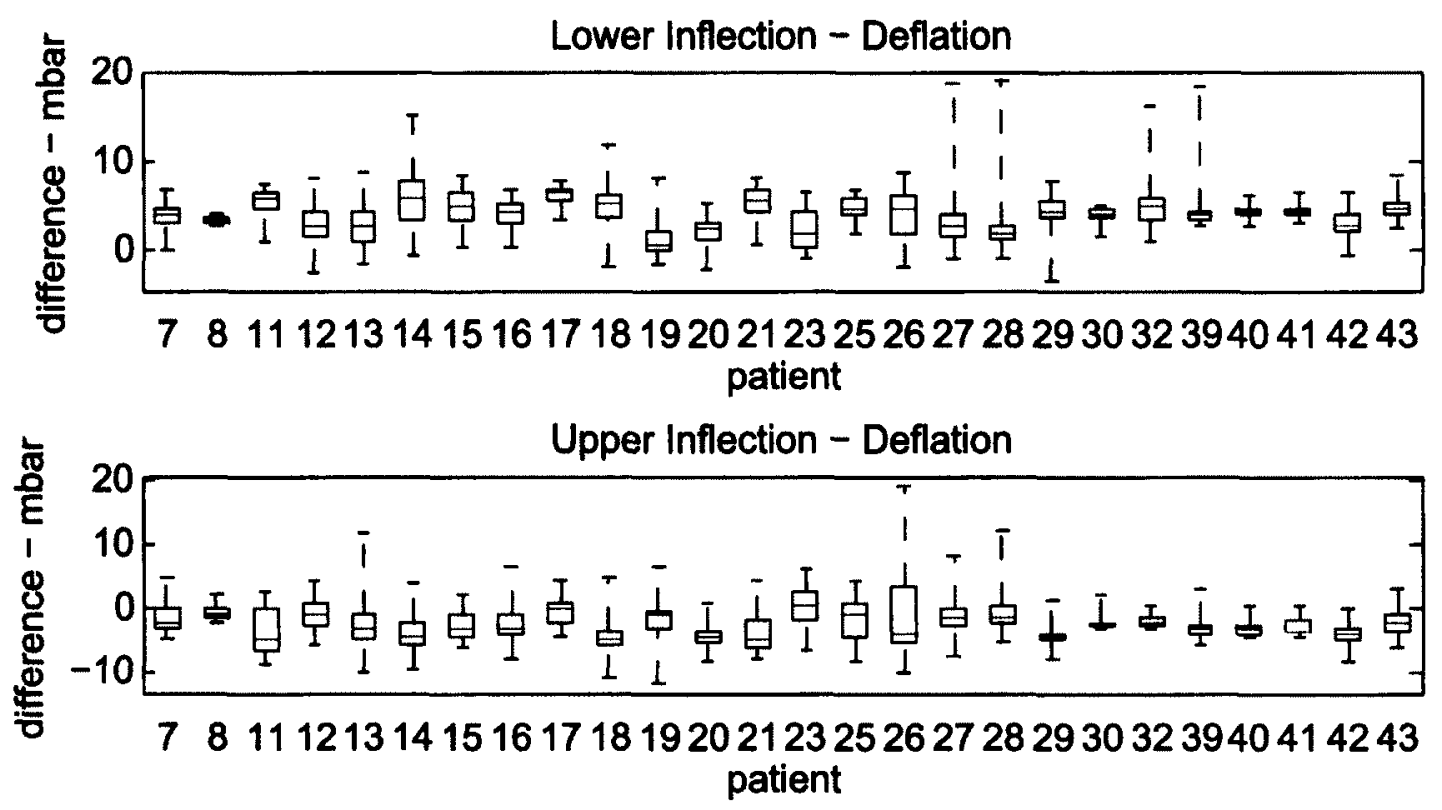

(b) Deflation Arm

Figure 8.2: Difference in IP between the Sigmoid and Linear methods. The boxes around the median line represent the $75^{\text {th }}$ to $25^{\text {th }}$ quartile while the dashed line represents the max and min for each difference. A lack of comparative data is indicated in Patients with no quartile boxes. N/A signifies patients in which neither algorithm was able to locate an IP in the same pixel. 
inflation limb and a $r_{\% \text { diff-lipdef }}=0.2018(p=0.3229)$ was found for the LIP on the deflation limb. These correlations were found by taking the Person correlation with a two-tail student $t$-test between the percent of pixels in which no IP was found verses the median difference for the entire lung. With 26 patients each vector had at max 26 values, with fewer due to no inflection points being found and thus no difference being calculated. The high p-value suggest that the number of pixels in the difference calculation have little effect on the actual difference value. Another idea which helps explain why there was a lack of samples for the LIP and UIP in the sigmoid method was that the airway pressure was limited to either $2 \mathrm{~L}$ tidal volume or $35 \mathrm{mbar}$ and in one patient the max pressure for the entire maneuver was as low as $19 \mathrm{mbar}$ and the minimum pressure being as high as 5.1 mbar. [Pulletz et al., 2011] also noted the $\max$ and min pressure problem and stated that the extent of the minimum/maximum pressure settings is unknown in clinical environments. Fig. 8.3 shows the distribution of the max and min pressures for the inflation limb and deflation limbs for all patients.
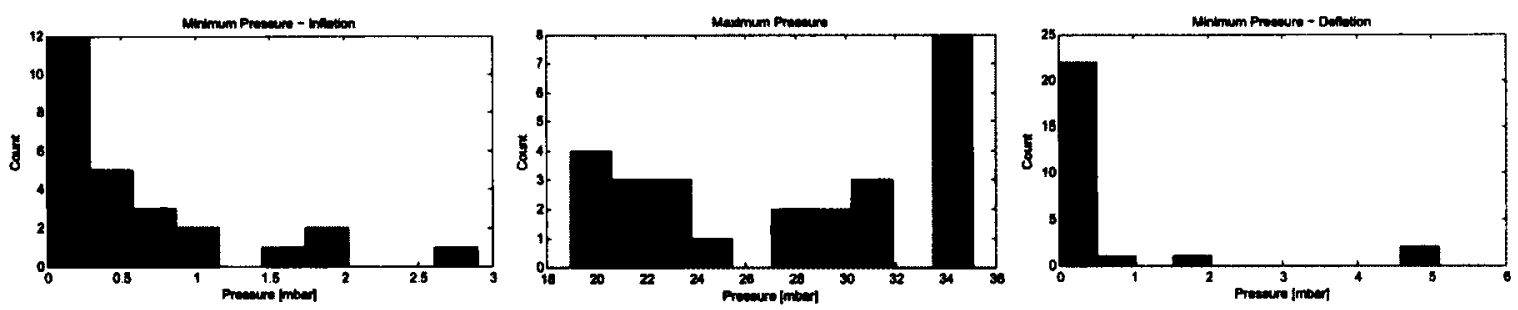

Figure 8.3: Histogram of the minimum and maximum pressures for all patients. The maximum pressure for the inflation and deflation limb are the same.

Testing to see if the max and min pressure had a significant relation on the differences (8.1) was used, where $d_{a}$ was the absolute difference between the IP and $p p$ is either the max or min pressure depending on if the UIP or the LIP respectively was being tested. It was found that a correlation did exist for the UIP on the inflation limb while none existed for the LIP on the deflation limb. The UIP on the inflation limb, which showed differences of $-6.80 \mathrm{mbar}$, had a correlation of 
$r_{m x m n-\text { diff-uipinf }}=0.4299(p=0.0518)$ with the max pressure while the difference for the LIP on the deflation limb had a $r_{m x m n-\text { diff-lipdef }}=-0.1072(p=0.6021)$ with the min pressure. From these results we can see a slight trend did exist between the differences in the UIP on the inflation limb and the maximum pressure used, while no correlation can be seen in the LIP on the deflation limb. This could be because of the narrow distribution of the min pressure in the deflation limb. The correlations can be seen in Table 8.2. Despite the very low p-value it can not be said that a relationship exists, further experimentation is needed to avoid any false classification [Ioannidis, 2005], but it does cater to the idea of further testing.

\begin{tabular}{|c|c|c|}
\hline & Correlation & p-value \\
\hline \hline \% Difference - UIP on Inflation & $r_{\% \text { diff-uipinf }}=0.0360$ & $p=0.8769$ \\
\% Difference - LIP on Deflation & $r_{\% \text { diff-lipdef }}=0.2018$ & $p=0.3229$ \\
Max Pressure - UIP on Inflation & $r_{m x m n-\text { diff-uipinf }}=0.4299$ & $p=0.0518$ \\
Min Pressure - LIP on Deflation & $r_{m x m n-\text { diff-lipdef }}=-0.1072$ & $p=0.6021$ \\
\hline
\end{tabular}

Table 8.2: Correlations used to explain large Inflection Point difference between the Sigmoid and Linear method

Another possible reason for such high differences could be due to the linear spline algorithm method of locating IP. Adjustments to how the linear method finds IP could be made to try and reduce the difference between the two methods. This alternative method has been implemented once before and has intuitive sense by trying to keep the first and last slopes to a low compliance [Grychtol et al., 2009]. This and more ideas are discussed in Chapter 9.

[Grychtol et al., 2009] compared the sigmoid method on a global scale to a threepiece linear method and found high correlations $\left(r^{2}=0.955, p<0.001\right)$ for both inflection points on post injury patients. A small difference between the spline and sigmoid method was found in the lower inflection point. An average of $1.43 \mathrm{mbar}$ was observed in the spline method over the sigmoid method and statistically tested 
with a paired sample T-test. [Grychtol et al., 2009]s result matches closely with the difference found in this thesis. In this thesis a 1.47 mbar average difference between the linear spline and sigmoid method was found.

Overall the difference between the IPs found by the two methods were similar and match previous results [Grychtol et al., 2009]. A major improvement with the linear method compared to the sigmoid method is the consistency in finding IPs. As mentioned earlier the linear method was able to locate an IP $99.95 \%$ while the sigmoid method was only able to locate IPs $57.13 \%$ of the time. The consistency found in the linear method allows for repeatable results over multiple experiments. The linear methods similarity to manual curve fitting also makes it a great fit for hospitals as clinicians will be familiar with workings and understand the results better then using the sigmoid method.

\subsubsection{Linear and Sigmoid vs. Visual Heuristics}

In this thesis we tested four pixels from each patient and asked five volunteers to fit three linear segments to the data. All volunteers except one had an Engineering background with the other having an English major background. The location of the inflection points were the points where the two lateral line segments intersected the median. An example of what each participant had to do is illustrated in (Fig. 8.4). The provided instructions were to fit the data in an optimal manner with three line segments. Each participant worked with both the inflation and deflation arms of the recruitment maneuver thus fitting eight pressure-impedance (PI) curves per patient. The experiment was designed in such a way that each person was only given one chance to place an IP thus medians over the participants were taken to help reduce the effects of a misplaced IP. It should be noted that each participant fitted the same PI curves providing continuity between participants and allowing for stats to taken 
over the participant data. For a detailed look of the location of the pixels refer to Fig. B.1.

$$
\text { Difference }=\text { Auto IP }- \text { Visual Heuristic IP }
$$



(a) Patient 15 - Pixel 4 - Good pixel

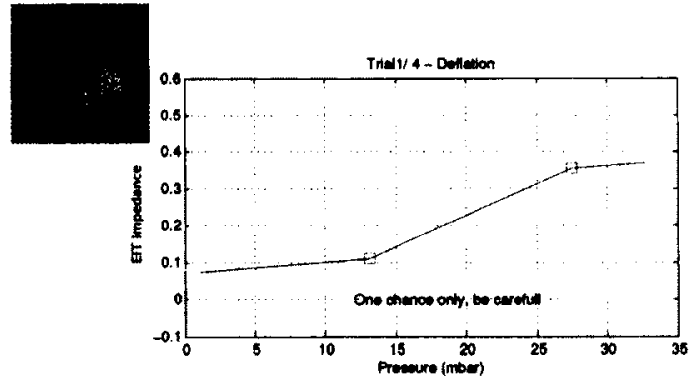

(b) Patient 14 - Pixel 1 - Bad pixel

Figure 8.4: Examples of both 'good' and 'bad' case scenarios encountered during the visual heuristic experiment. The PI data is taken from the pixel shown in the EIT image in the top left corner.

\section{Linear vs. Visual Heuristics}

In order to compare the linear and visual methods (8.2) was used and the median was found over the 5 participants then averaged over the 26 patients and 4 pixels. Similar inflection points were found between the linear and visual heuristic methods with the difference being -0.6247 mbar for the LIP and -0.4662 mbar for the UIP. Expanded results looking into per pixel differences can be seen in Table B.1. Comparing the IP generated by the linear spline method and the visual heuristic method provides some insight to the accuracy of the linear based results since prior to function based curve fitting inflection points were located by eye [Matamis et al., 1984a, Martin-Lefevre et al., 2001].

Fig. 8.5 and Table B.1 shows the difference between the human inflection points and linear based inflection points. From the table we can see the differences are quite 

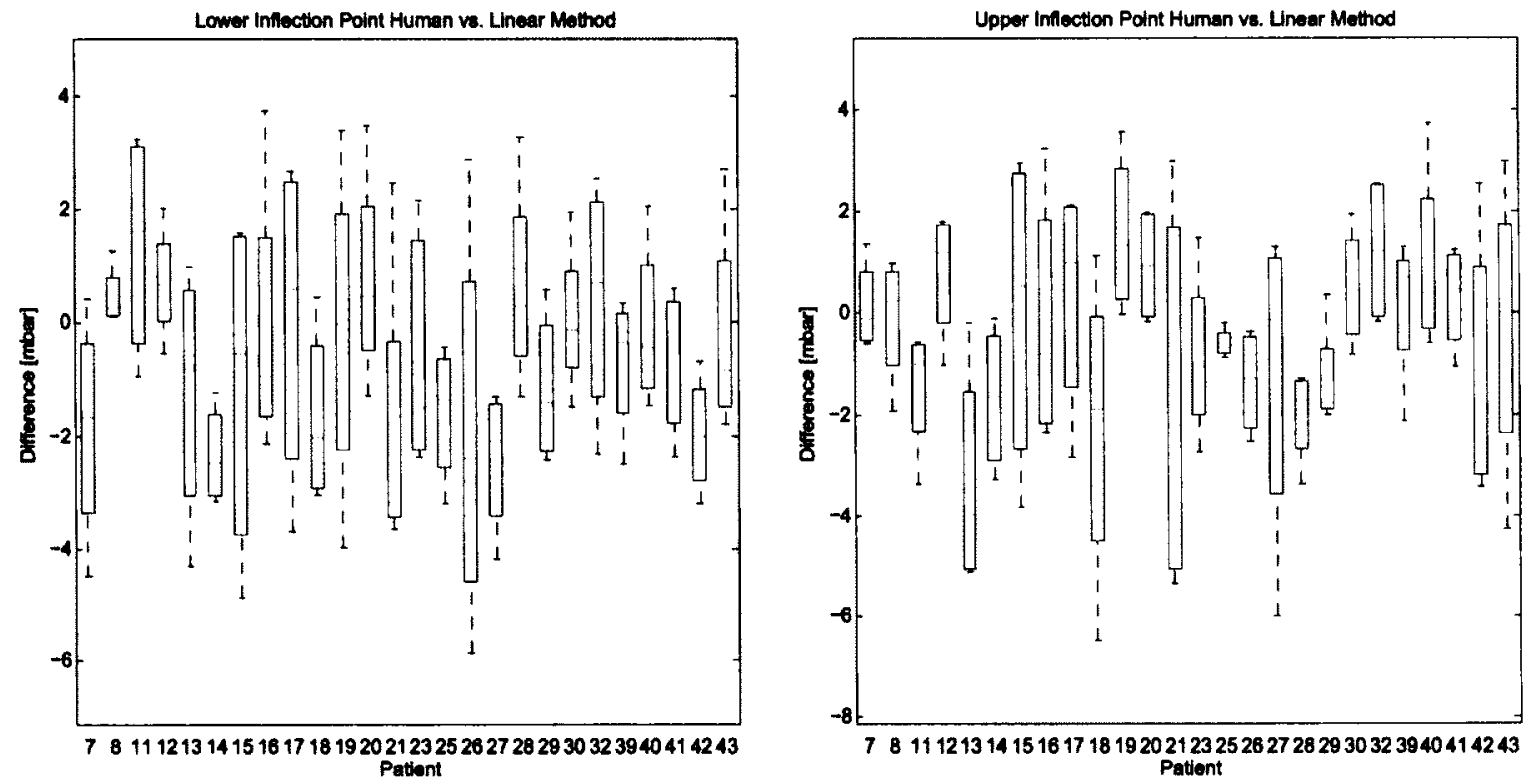

Figure 8.5: Box plot showing the difference in IP from the human heuristics method and the linear spline method. The boxes around the median line represents the $75^{\text {th }}$ to $25^{\text {th }}$ quartile while the dashed lines represents the max and min for each difference.

small with the best average difference being 0.007 mbar and a median difference of -0.0240 mbar. The median and average were taken over all patients. These values were made from the upper inflection of the 2 ind pixel which has the common location throughout all patients for being on the right lateral side when the anterior side is up. With the majority of patients being in the medial anterior - posterior plane. For a detailed look at the location of each pixel for all patients refer to Fig. B.1.

\section{Sigmoid vs. Visual Heuristics}

Similar to the linear comparison (8.2) was used to compare the sigmoid method and visual heuristic method with the median being taken over the 5 participants then averaged over the 26 patients and 4 pixels. Larger differences were noticed with sigmoid method, compared to the linear vs. visual comparison. Differences of 2.09 mbar for the LIP and $2.6 \mathrm{mbar}$ for the UIP were found. Additional to the larger differences the sigmoid method was again unable to find many IP. For this comparison 
a total of 147 of a possible 208 IP were not found, this is $70.7 \%$. In Fig. 8.6 N/A represents no comparison was made due to a lack of IP found by the sigmoid method. Plots showing a single red line indicates only 1 of 4 IP were found by the sigmoid method.


Figure 8.6: Box plot showing the difference in IP from the human heuristics method and the linear spline method. The boxes around the median line represents the $75^{\text {th }}$ to $25^{\text {th }}$ quartile while the dashed lines represents the max and min for each difference. A lack of comparative data is represented by no quartile box surrounding the median. N/A means the sigmoid method was unable to find an inflection points for any of the four pixels.

\subsubsection{Conclusion}

Overall from the larger differences between the visual heuristic and sigmoid method as well as the lack of finding inflection points makes the linear spline method a better choice. With more testing the linear spline method can be compared to a larger sample of IPs found from visual heuristics by professionals. From these tests though it is recommended that the linear spline method be further looked into. 


\subsection{Membership Graph and IF-THEN Rules}

In this section details of past Fuzzy Logic Systems (FLS) and current Fuzzy Logic Systems for the task of lung segmentation and classification are discussed. Each FLS is analysed using the [Wu and Mendel, 2011] criteria and can be seen in Table 8.3.

\begin{tabular}{l|l} 
Validity: & $\begin{array}{l}\text { The summaries must be derived from data with high confidence. } \\
\text { This describes how much of the data supports the summaries. } \\
\text { Usefulness: }\end{array}$ \\
This relates the summaries to the goal of the user, especially in terms of \\
the impact that these summaries may have on decision-making. \\
This describes the degree to which the summaries deviate from our ex- \\
Simplicity: & $\begin{array}{l}\text { This measure concerns the syntactic complexity of the summaries. Gen- } \\
\text { erally, simpler summaries are easier to understand and, hence, are pre- } \\
\text { ferred. }\end{array}$
\end{tabular}

Table 8.3: [Wu and Mendel, 2011] criteria for testing IF-THEN systems.

Using IP as the integral part of the fuzzy membership is a new idea when it comes to automated fuzzy PEEP setting for mechanical ventilation.

[Grychtol et al., 2010]s FLS base classifier fuzzified its inputs to three fuzzy sets: Low, Average, and High. In [Grychtol et al., 2010] the membership graphs were trapezoidal and spanned from the minimum value to the maximum value of each pixel with the mean being right in the middle of the Average fuzzy set. This method of using three fuzzy sets and many features relied on its rules to perform the segmentation. The system by [Grychtol et al., 2010] used more complicated rules using the impedance change values, the spectral information of the impedance change, and the pressure as linguistic variables to be fuzzified.

Using the [Wu and Mendel, 2011] criteria for quality of IF-THEN systems [Grychtol et al., 2010] was analysed. The rules implemented in [Grychtol et al., 2010] performed well with few improvements. The rules summarized the data well but were missing a conclusion for normal states. Instead [Grychtol et al., 2010] deciding on 
using the transition states such as; Collapsing, Opening, Recovering and Overdistending. The implemented rules were useful in achieving the goal of classification and do provide novelty; but, when simplicity is a concern, this set of rules are quite complicated. An example of one of the rules in [Grychtol et al., 2010] can be seen below.

$$
\begin{gathered}
\operatorname{Opening}(n)=(f e i t(n)=L o) \cap(f e i t(n-1)=L o) \cap(\operatorname{sgn}(\Delta f e i t(n))=H i) \\
\cap(\operatorname{sgn}(\Delta \sigma(n))=L o) \cap(\operatorname{sgn}(\Delta p)=H i)
\end{gathered}
$$

Similar to [Grychtol et al., 2010] this thesis has satisfied many of [Wu and Mendel, 2011] criteria. Validity: The rules in the FLS system were very closely tied to the data as they used the inflection points and knowledge of how PEEP was set in previous studies to create the membership which intern lead to the rules. Generality: This criteria was harder to asses as no hard validation data was available. While the data used created the membership graphs and rule base it can not be said if the data supports the truth of the rules. Since the IP were an integral part in the creation of the membership graphs if the IP were miscalculated the error would propagate into the FLS thus determining where the error originates impertinent. Usefulness: The rules in this FLS were extremely useful with no redundant or useless rules. Novelty: The rule base is novel as including the IP in a FLS has not been done before but the rules were largely based on knowledge attained from previous global based PEEP settings. An overview of past PEEP settings are discussed in Chapter 6 . Simplicity: The rule base in this study were extremely simple. 


\subsection{Optimal PEEP}

The optimal PEEP in this thesis was found using two separate systems $\left(F S_{p}\right.$ and $F S_{e}$ ), with the difference being the linguistic variables. The first, $F S_{p}$, used pressure as the input variable while the second, $F S_{e}$, used impedance change values. Details on the design of the algorithm are discussed in Chapter 7. Fig. 8.7 demonstrates the progression from collapsed states to overdistended states, from the figure it's clear to see some pressure ranges have larger areas of normal states.

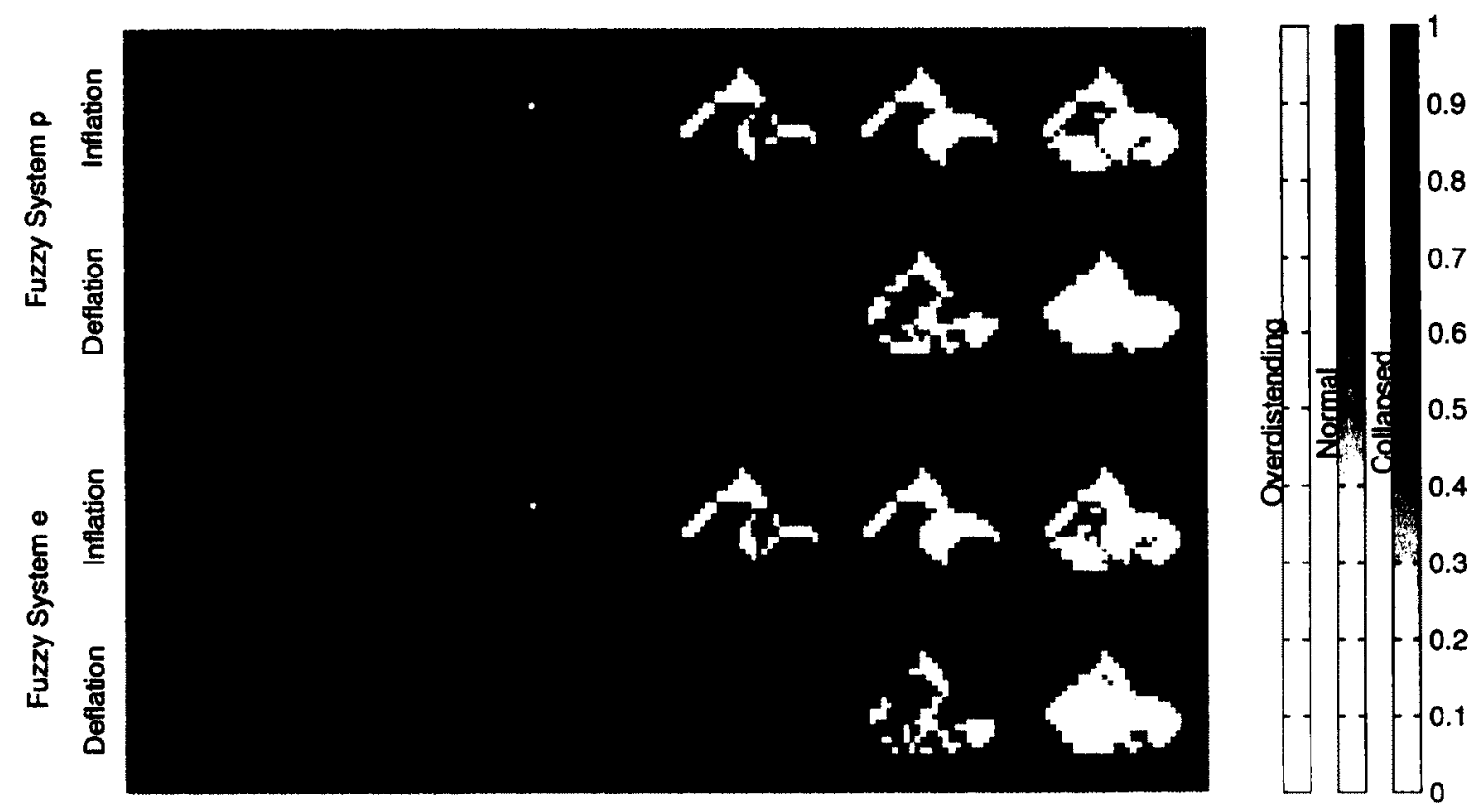

Figure 8.7: The progressive change of lungs regions as the pressure increases and decreases. The classifications are derived from the fuzzy logic system and show the progressive change from collapse to overdistended as the pressure increases. This figure also demonstrates the heterogeneous nature of the lungs.

Comparing the two FLS it can be seen that the results for optimal pressure were very similar. The average difference between the two systems was $0.74 \mathrm{mbar}$ and $-0.38 \mathrm{mbar}$ for inflation and deflation limbs respectively. The difference is illustrated in Fig. 8.8. The median difference between the two systems was 0.7 mbar and -0.5 mbar with the $\sigma$ being 0.98 mbar and 1.42 mbar for inflation and deflation respectively. 
The tabulated results are shown in Table 8.4. The average PEEP for both methods were 12.9 mbar and $16.6 \mathrm{mbar}$, and $12.1 \mathrm{mbar}$ and $19.9 \mathrm{mbar}$ for $F S_{p}$ and $F S_{e}$, and inflation and deflation respectively.

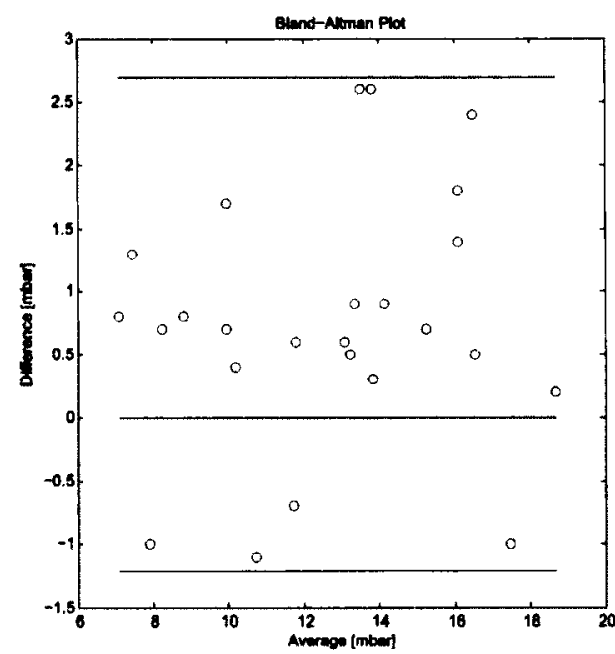

(a) Inflation



(b) Deflation

Figure 8.8: Difference and average of the optimal PEEP found between $F S_{p}$ and $F S_{e}$

\begin{tabular}{|c|c|c|}
\hline Statistic & Inflation & Deflation \\
\hline \hline Mean & 0.74 & -0.38 \\
Std & 0.98 & 1.42 \\
Median & 0.70 & -0.50 \\
\hline
\end{tabular}

Table 8.4: Results of the difference comparison for the optimal PEEP between Fuzzy Rule System p and e

[Takeuchi et al., 2002] showed that from a global PV curve the best PEEP to use was LIP +2 mbar. Using this idea a global pressure-impedance curve was created and the LIP +2 mbar was found. Comparing this value to the calculated optimal PEEP from $F S_{p}$ and $F S_{e}$ we can see that the FLS obtain similar results for some patients but not with others, Table 8.5 and Fig. 8.9.

From Fig. 8.9 four sample patients were selected for further analysis. Two patients with large error and two patients with near zero error. In Fig. 8.11 we can see the 


\begin{tabular}{|c|c|c|c|c|}
\hline Statistic & \multicolumn{2}{|c|}{ Fuzzy System p } & \multicolumn{2}{c|}{ Fuzzy System e } \\
& Inflation & Deflation & Inflation & Deflation \\
\hline \hline Mean & 2.70 & 6.68 & 1.96 & 7.06 \\
Std & 10.60 & 27.89 & 10.58 & 23.12 \\
Median & 1.98 & 7.20 & 1.15 & 7.37 \\
\hline
\end{tabular}

Table 8.5: Results of the comparison between Fuzzy Rule system $\mathrm{p}$ and e with [Takeuchi et al., 2002] result of LIP +2 mbar as a good PEEP.

global PI curves with the linear fit and inflection points and the FLS optimal selection graph. Looking at Fig. 8.11b we can see how the large difference between the two systems arrived. The LIP on the global graph is largely dependent on the curve fitting algorithm thus an errors in linear spline method will be weighted greatly on the PEEP selection. For the Fuzzy Logic system each pixel is fitted by the linear spline. Comparing patient 12 and 16 to the human heuristic method large variability can be seen. This is illustrated in Fig. 8.10. While patient 8 and 17 have low variability. This shows the IP calculation needs to be tweaked for increased accuracy.

\subsection{Conclusion}

Overall the algorithm did what it was designed to do, locate and suggest a PEEP based on regional data obtained from EIT. In terms of success with no gold standard to compare to, it is difficult to say if the suggested PEEP will produce better results then current methods. In terms of the suggested PEEPs both systems performed well with very little difference between the two the systems. I would suggest using the pressure based system as it is easier to follow and provides smoother input signals.

Between choosing the deflation limb verse the inflation limb for PEEP selection. [Hinz et al., 2006, Bigatello et al., 1999] have suggested the use of the deflation limb as collapsed lung tissue recruit at a higher PEEP then is needed to maintain open 
tissue. In this thesis opposite results were noticed, as shown in Fig. 8.12. This can be explained since prior to the recruitment maneuver ventilated breathing of the patient was taking place. This ventilation could have already initially recruited the tissue.

Drawing conclusions from the comparisons it can be said that the linear method for locating inflection points from pressure-impedance graphs is worth using. The linear method allows for locating inflection points reliably. It's also easy to understand by doctors, providing easy to access standards. It also obtained similar results to the visual heuristic method showing it's accuracy. It also had similar results to that of the Sigmoid method which is known to be a classical method for locating IP.

The automated PEEP portion of the work also worked well. It was able to suggest a PEEP using regional IP and knowledge of the use of PEEP and the effect on lung tissue at various PEEPs. The designed system also has benefit to be used to control mechanical ventilation systems. 

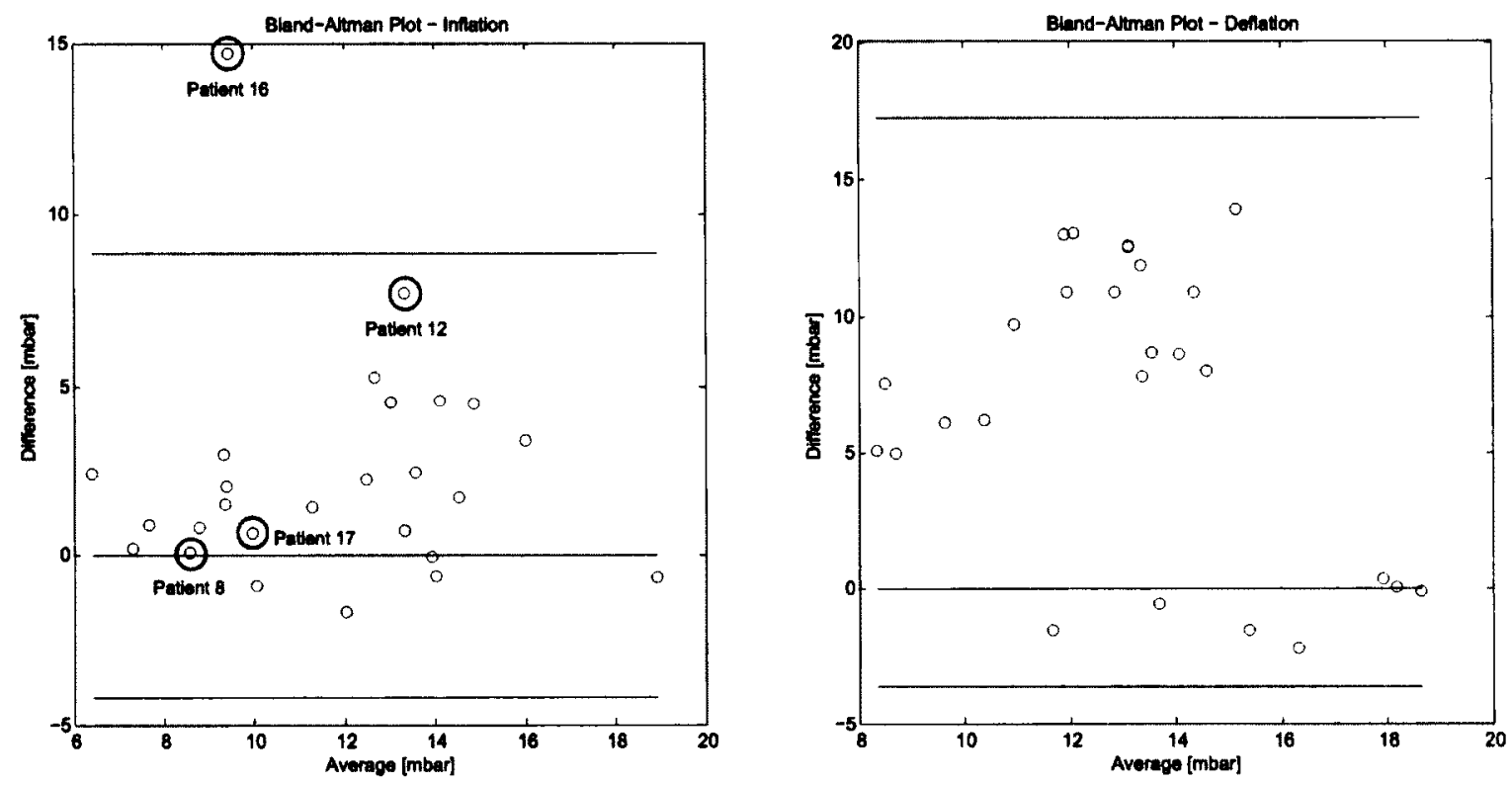

(a) Fuzzy Logic System $p$
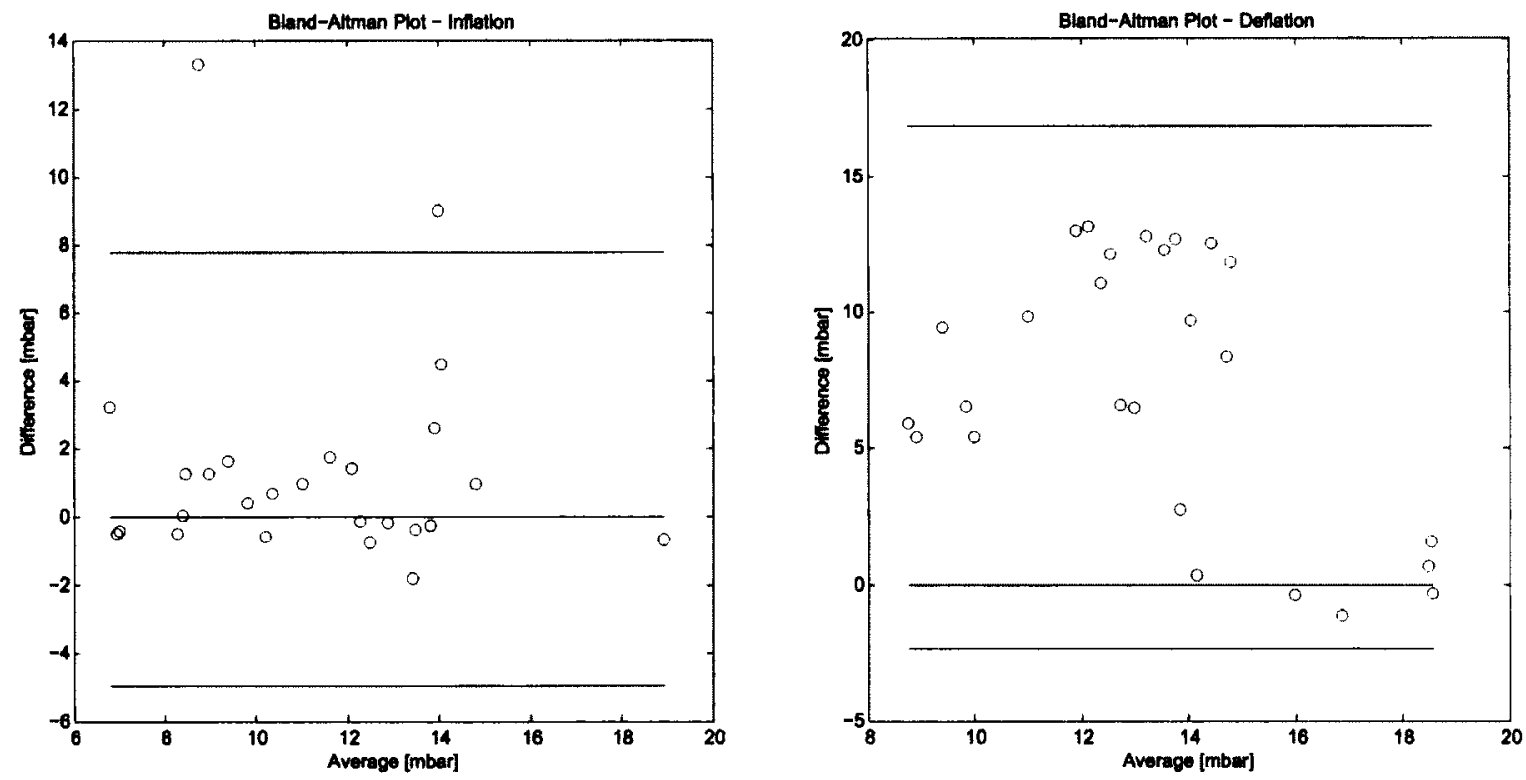

(b) Fuzzy Logic System e

Figure 8.9: Difference and average of the optimal PEEP for both FLS and [Takeuchi et al., 2002] suggested PEEP of using LIP +2 mbar. Within Fig. $8.9 \mathrm{~b}$ the circles indicate further analysis was performed. 




Figure 8.10: Difference between the human heuristic method and the linear spline method for the lower inflection point on the inflation limb only. 

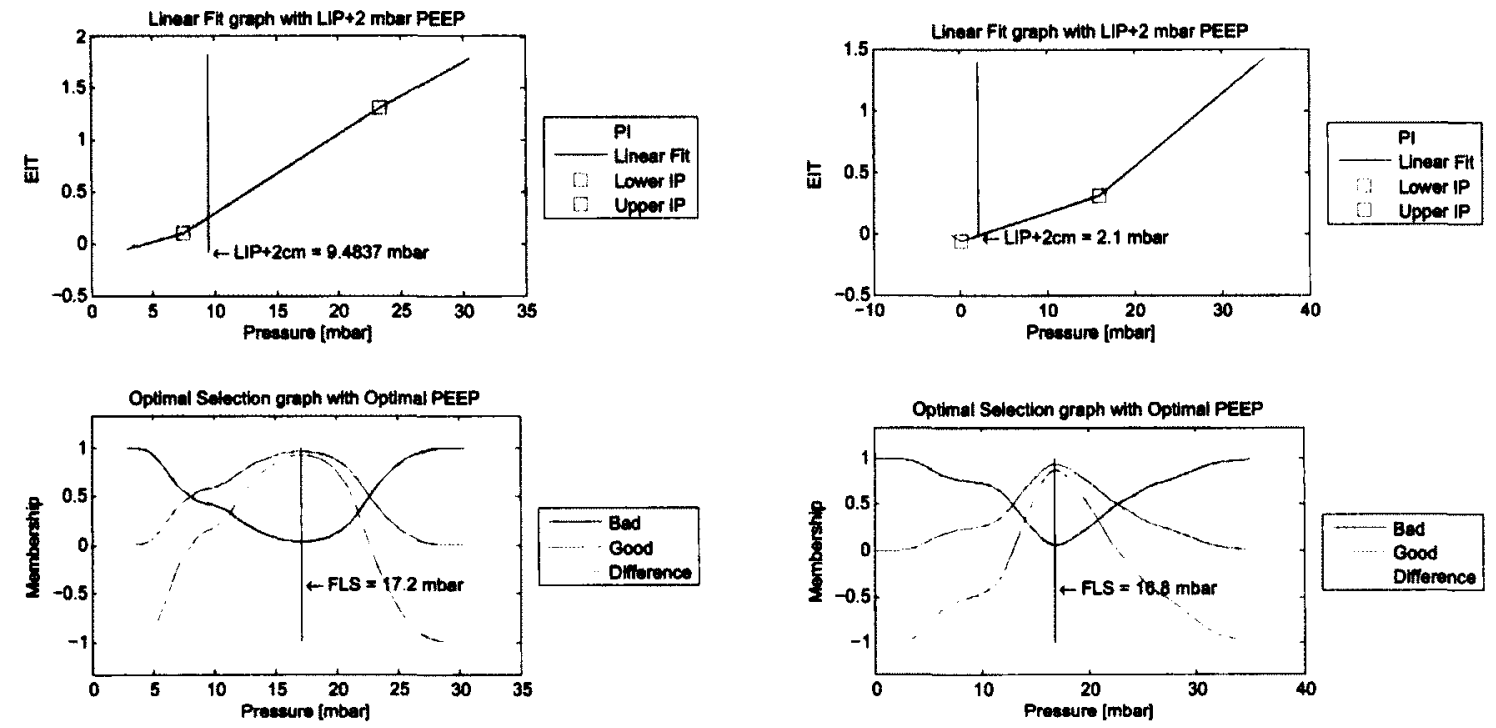

(a) Patient 12

(b) Patient 16
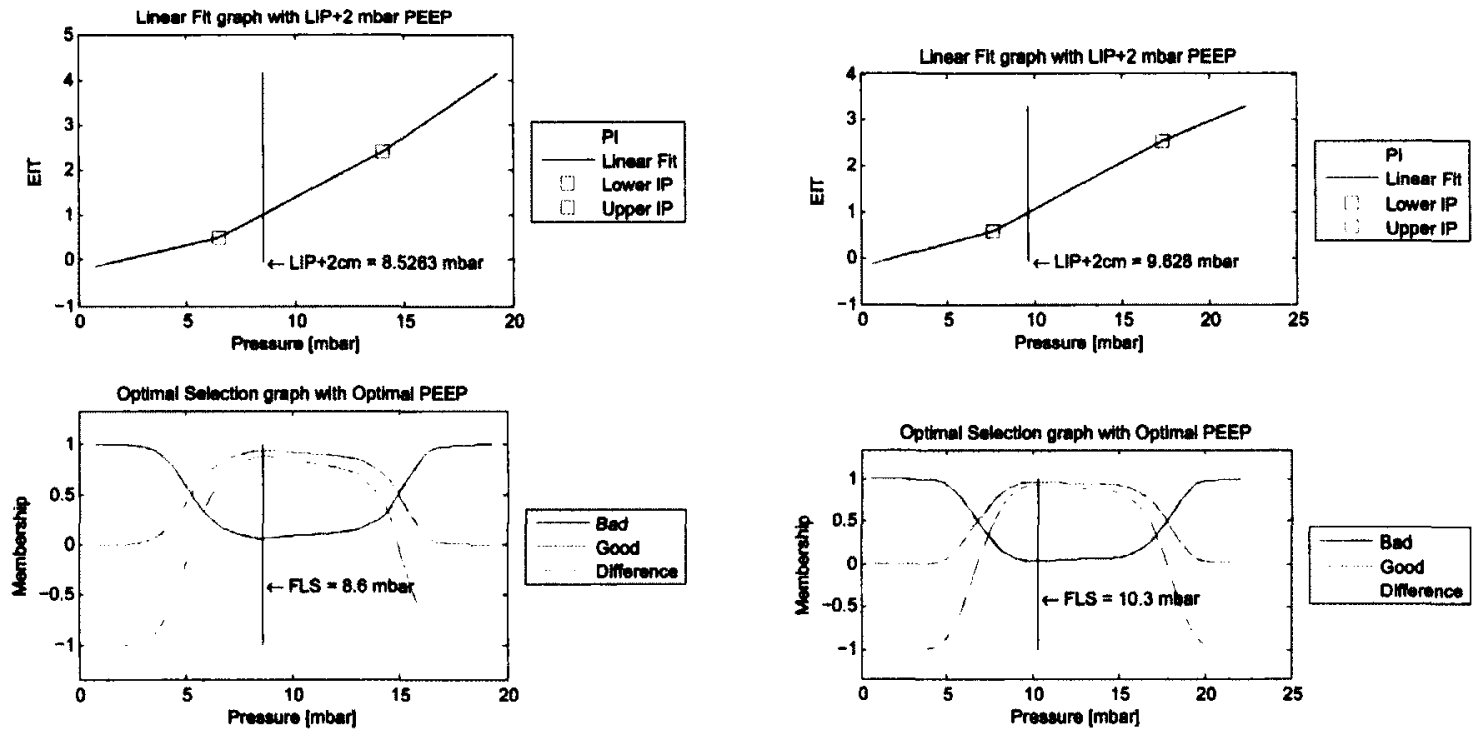

(c) Patient 8

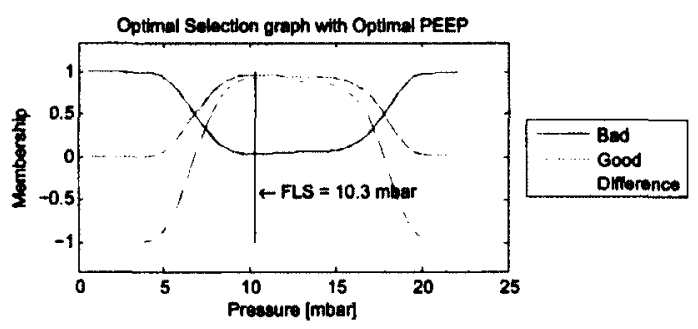

(d) Patient 17

Figure 8.11: Global PI curve with LIP, UIP, and the LIP+2 mbar pressure and the Fuzzy optimal selection with according FLS based pressure. 


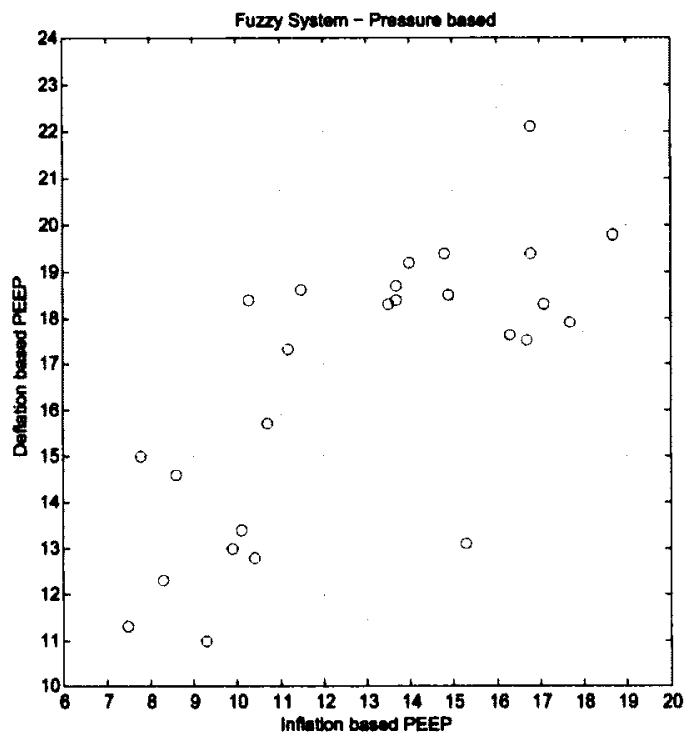

(a) FLS Pressure Based

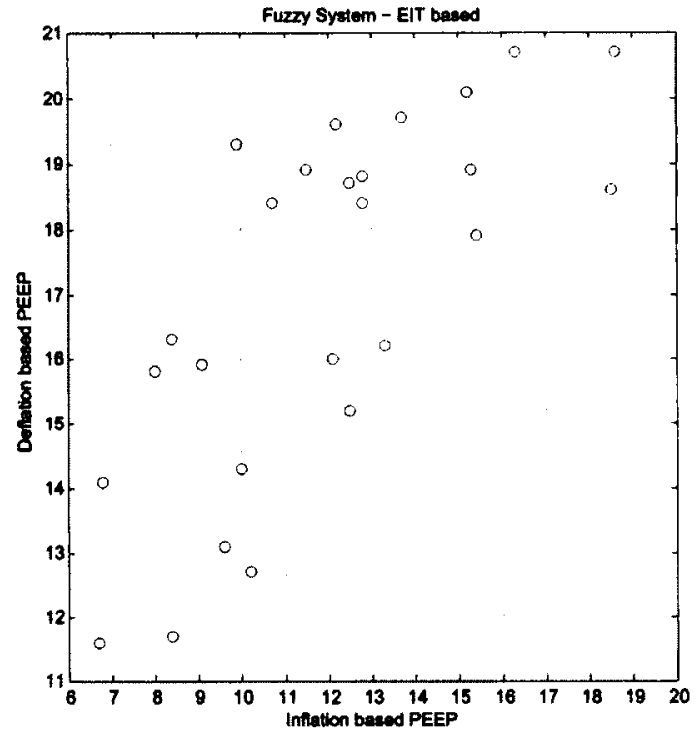

(b) FLS EIT based

Figure 8.12: The PEEP suggested using both Pressure based and EIT based systems. Each data point represents a patient. From the figure it can be seen the inflation limb suggested PEEP is lower compared to respective deflation suggested PEEP. 


\section{Chapter 9}

\section{Summary and Future Work}

This work takes a novel step toward development of tools for automated PEEP selection for mechanical ventilation systems. Using a quasi-static pressure based recruitment maneuver optimal PEEP was located to reduce lung injury conditions while maximizing typical working lung tissue. Contributions to this task were locating regional Inflection Points (IP) to reduce collapsed and overdistended lung tissue known to be factors of Ventilator Induce Lung Injury (VILI). Refer to Chapter 6 for a detailed study on locating IP. Additionally, using the lower and upper inflection points (LIP and UIP) a Singleton Type-1 Fuzzy Logic System (FLS) was created to classify lung regions as collapsed, normal, or overdistended. With the classifications a PEEP was located. Details on the algorithm design can be seen in Chapter 7 .

Evaluations were performed by comparing the recommended PEEP from this thesis to the recommended value in the study conducted by [Takeuchi et al., 2002]. Details of the comparison can be seen in Chapter 8.3.

This work investigates the use of regional IP along with the tool used to locate them, the linear spline technique [Grychtol et al., 2009]. It also investigates the use of Fuzzy Logic in converting quantitative values and expert knowledge of mechanical 
ventilation systems for ALI patients into classifications. This work retrospectively evaluated a group of 26 patients of which consisted of control and ALI patients. For more information on the data used refer to [Pulletz et al., 2011] or Chapter 4.

\subsection{Future Work}

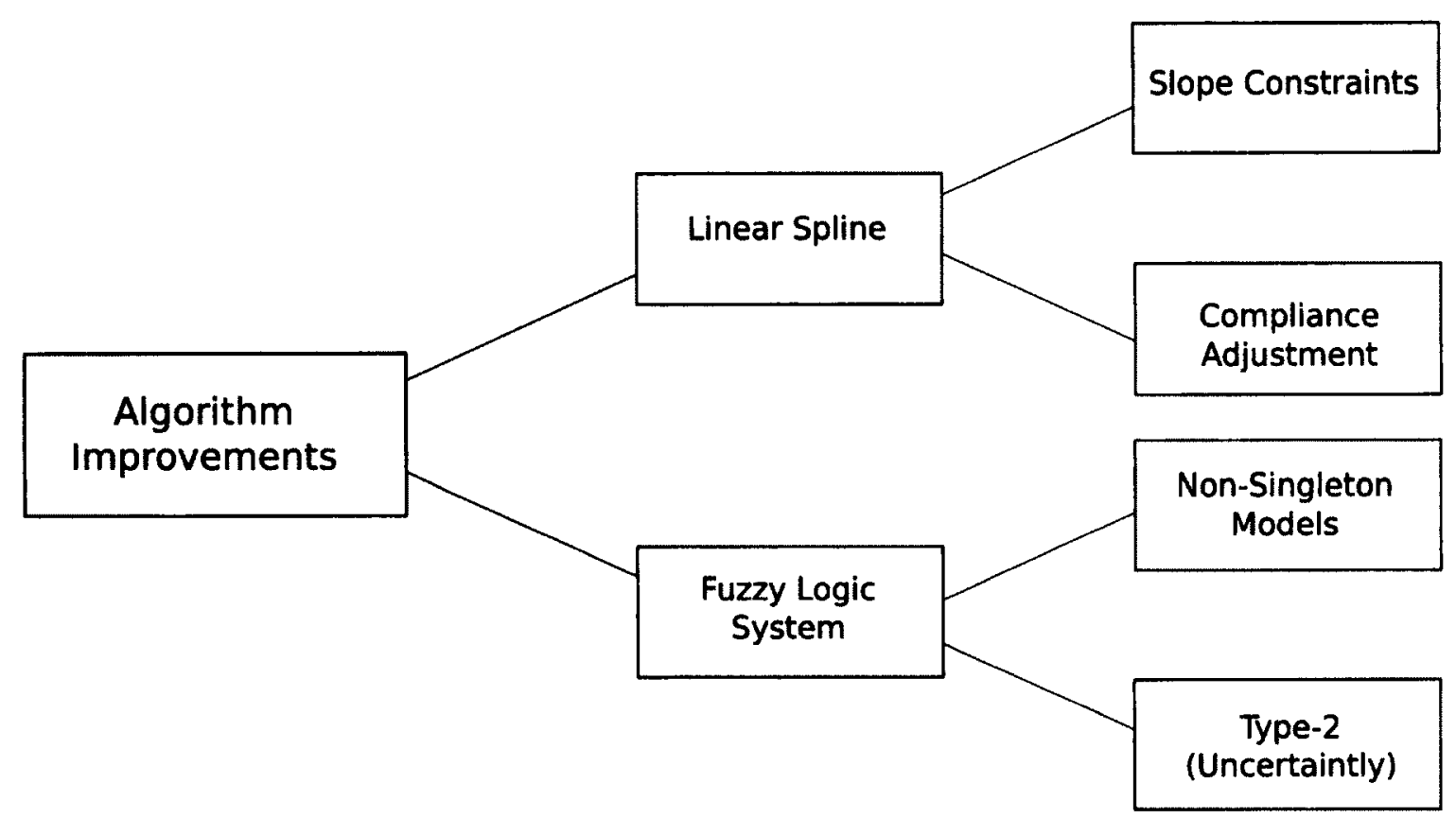

Figure 9.1: Algorithm improvement Tree Chart

\subsubsection{Linear Spline Improvements}

Improvements to the designed algorithm are broken into two categories and are illustrated in Fig. 9.1. The first improvement has to do with the algorithm used to detect the inflection points. The method currently implemented by [D'Errico, 2011] worked well and only needs minor revisions. Restrictions on the first and last line segment should be implemented to ensure relatively horizontal slopes are met. This 
would enforce the idea that these regions have low compliance and indicate collapsed or overdistended tissue. The current implementation locates the global optimal breakpoints and has no restrictions on the slopes (compliance). An additional improvement would be to convert the pressure-impedance graphs into standard pressure-volume graphs using the scaling factor in (9.1), where $P$ is the pressure, $V$ is the volume, and $\Delta Z$ is the change in impedance. Using (9.1) we could obtain regional pressure-volume curves making it similar to what past research [Harris, 2005, Papadakis and Lachmann, 2007, Lu et al., 1999, Takeuchi et al., 2001, Venegas et al., 1998, Martin-Lefevre et al., 2001, Harris et al., 2000].

$$
\frac{d V}{d P}=\frac{d V}{d \Delta Z} \frac{d \Delta Z}{d P}
$$

\subsubsection{Fuzzy Logic Improvements}

The second improvement is for the Fuzzy Logic System and is separated into two parts. The first is to incorporate uncertainty measures from the initial crisp data by apply non-singleton functions to the fuzzification processes. This works by modeling the data to a certain distribution, such as Gaussian, and then combining the distribution with the membership graph and choosing the largest value as the membership value. From Fig. 5.1 the variable $\mu_{X_{i}}\left(x_{i}\right)$ would the distribution. The second improvement would be to incorporate uncertainty measures into the fuzzy membership graphs. This would turn the Type-1 FLS into a Type-2 FLS. [Mendel, 2001] provides excellent documentation on this process and is a great source to Fuzzy Logic. The uncertainty measures can come from the variance incorporated within the EIT reconstruction.

$$
P_{A O}=\frac{V}{C}+\dot{V} R+\ddot{V} I-P_{m u s}
$$


A more novel improvement would be to tackle the assumption that the airway pressure which is used with in the pressure-impedance calculations are the same throughout the entire thorax [Costa et al., 2009].

In [Harris, 2005] the equation for airway pressure is used to model the changes in pressure of the respiratory system. We can see this equation in (9.2) where $V$ is the volume, $C$ is the respiratory compliance, $\dot{V}$ is the volume flow, $R$ is the respiratory resistance, $\ddot{V}$ is the volume acceleration, $I$ is the respiratory impedance, and $P_{m u s}$ is the pressure needed for respiratory muscle movement. All the variables listed assume equal lung expansion as can be seen from the globular nature.

For quasi-static and static recruitment maneuvers all factors other than $V$ and $C$ are ignored or negligible. The reason behind this is discussed in Chapter 2. Using the regional information of EIT, the compliance conversion equation in (9.1), and the measured volume provided by the mechanical ventilation system we can calculate the regional compliance. Using the regional compliance and measured volume we can calculate regional pressure values which can then by used for regional pressureimpedance curves.

\subsubsection{Future Testing}

With the lack of a golden standard in the data this study was only able to compute an optimal PEEP with no ground truth. With this being said a detailed study should be conducted in which patients are monitored after apply this algorithms suggested PEEP. From here we can record the survival rates along with the weaning time. Comparing this set of patients to a control a sense of long term results can be obtained. In terms of short term results CT scans can be taken and segmented into overdistended and collapsed regions, from here comparison between the algorithm in this thesis and the results from the CT scans can be compared. Finally arterial 
oxygen partial pressure, lung inflammation, and mRNA expressions can be taken at the suggested PEEP and compared to other recommendations.

Overall this thesis sets out to develop an automated system to analyse EIT images for the use of optimal PEEP selection. Its major contribution are: a) the testing of the linear spline method for the detection of inflection points within pressure-impedance curves, and b) the development of a fuzzy logic classification system. It is hoped that the contributions in this thesis will inspire future work for the improvement of patient care. 


\section{Appendix A}

\section{MATLAB Code}

All code in this section was written and designed by [D'Errico, 2011]. With his permission it was included in this thesis as it no longer appears online.

\section{A.1 Linear Spline}

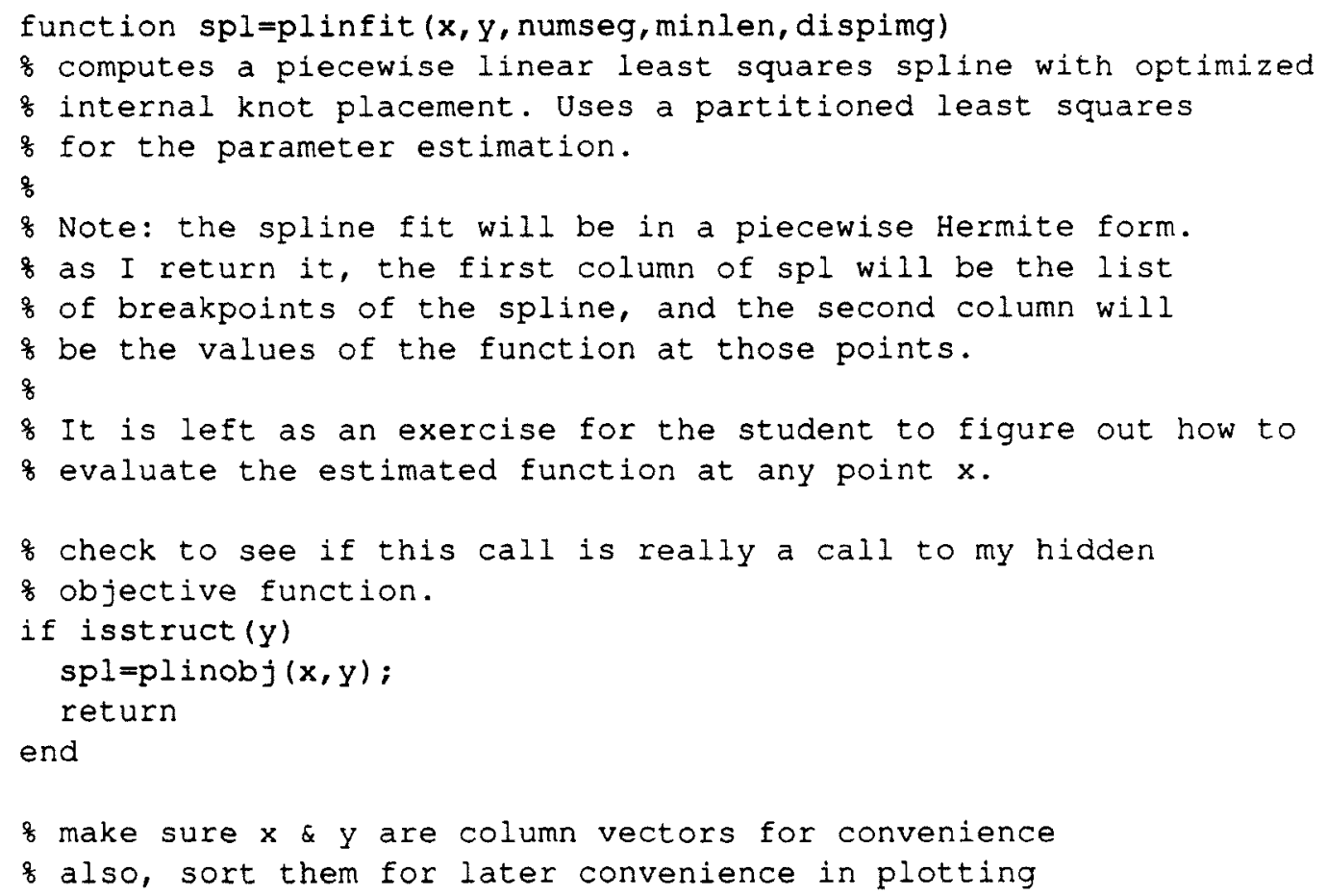




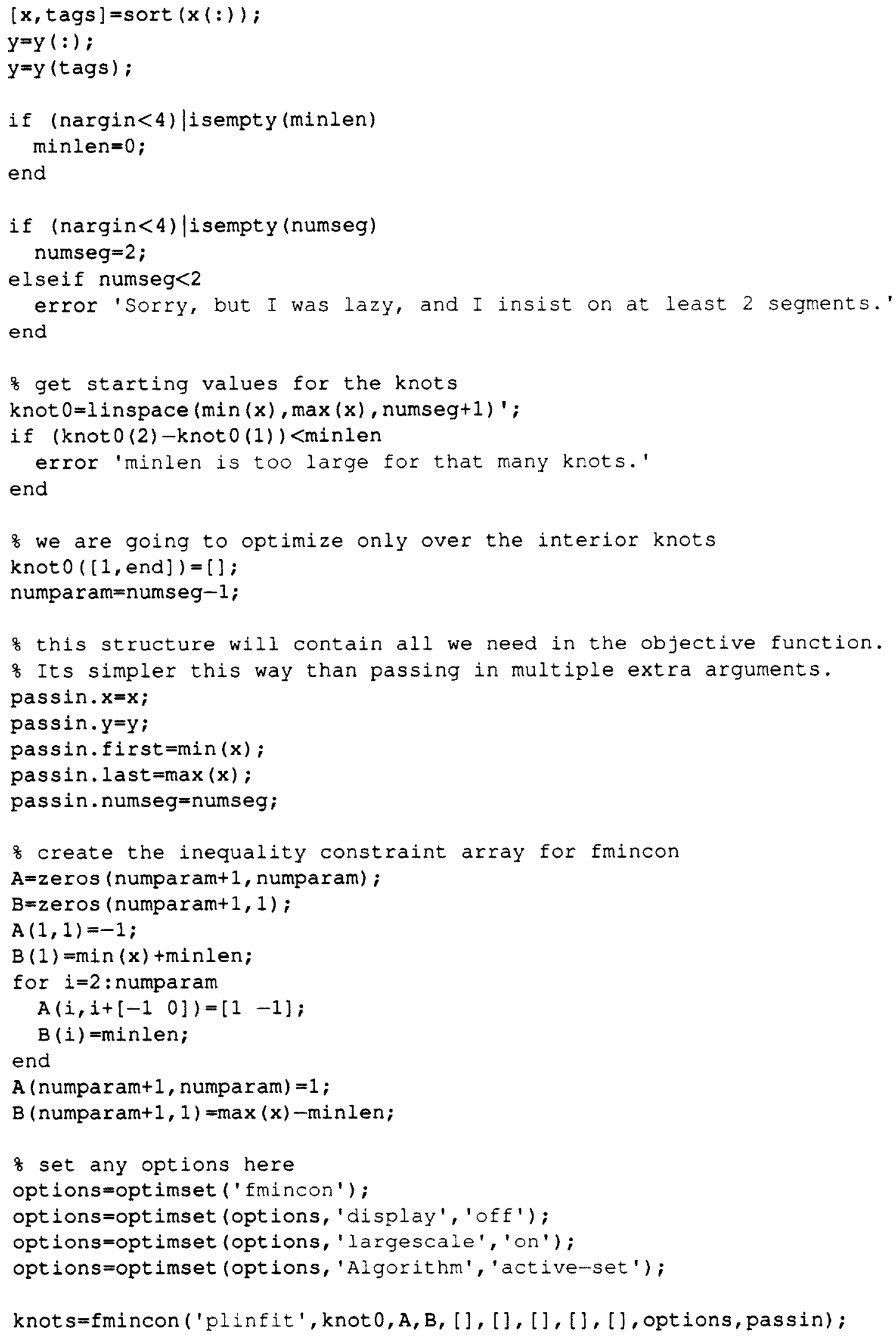




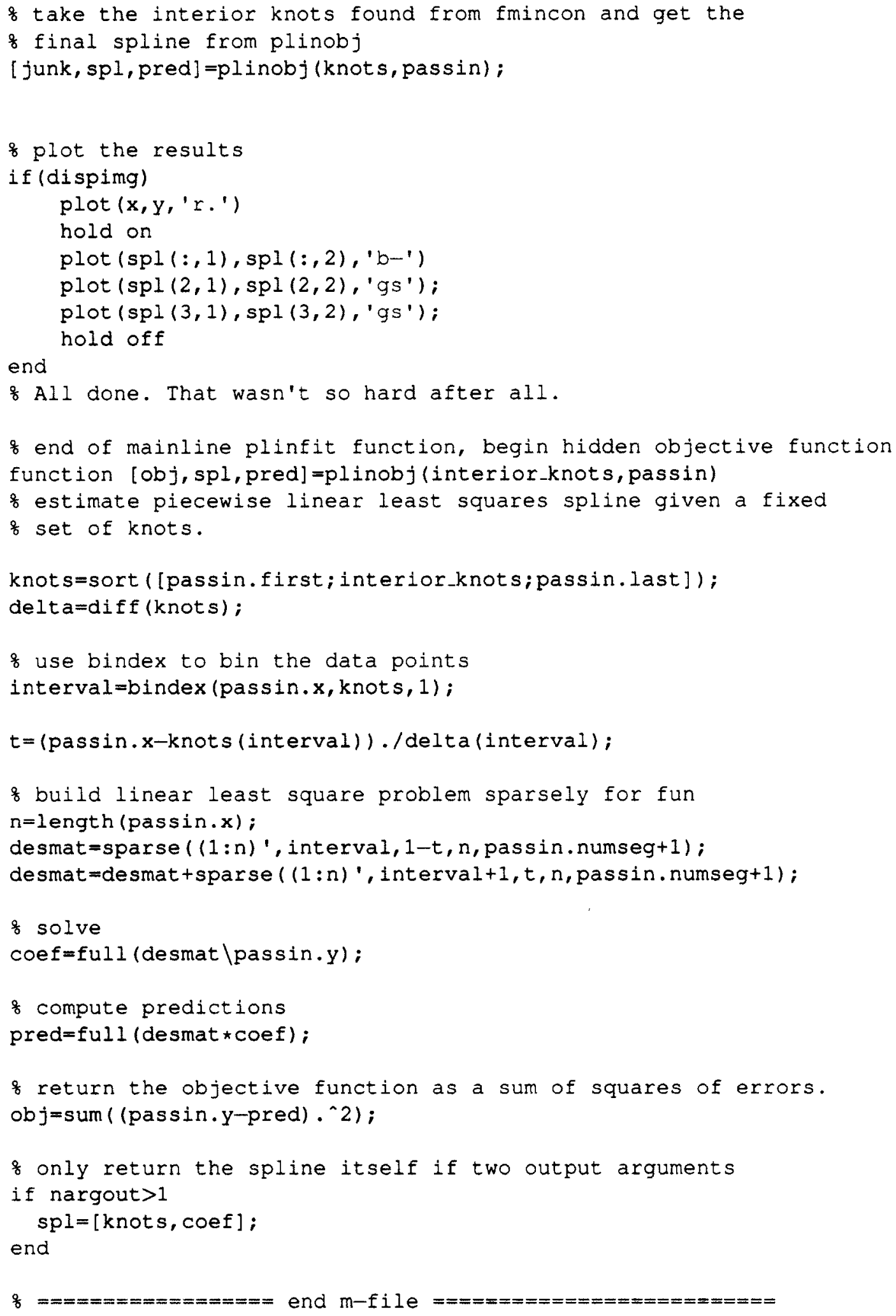




\section{A.1.1 Bin function needed for Spline}

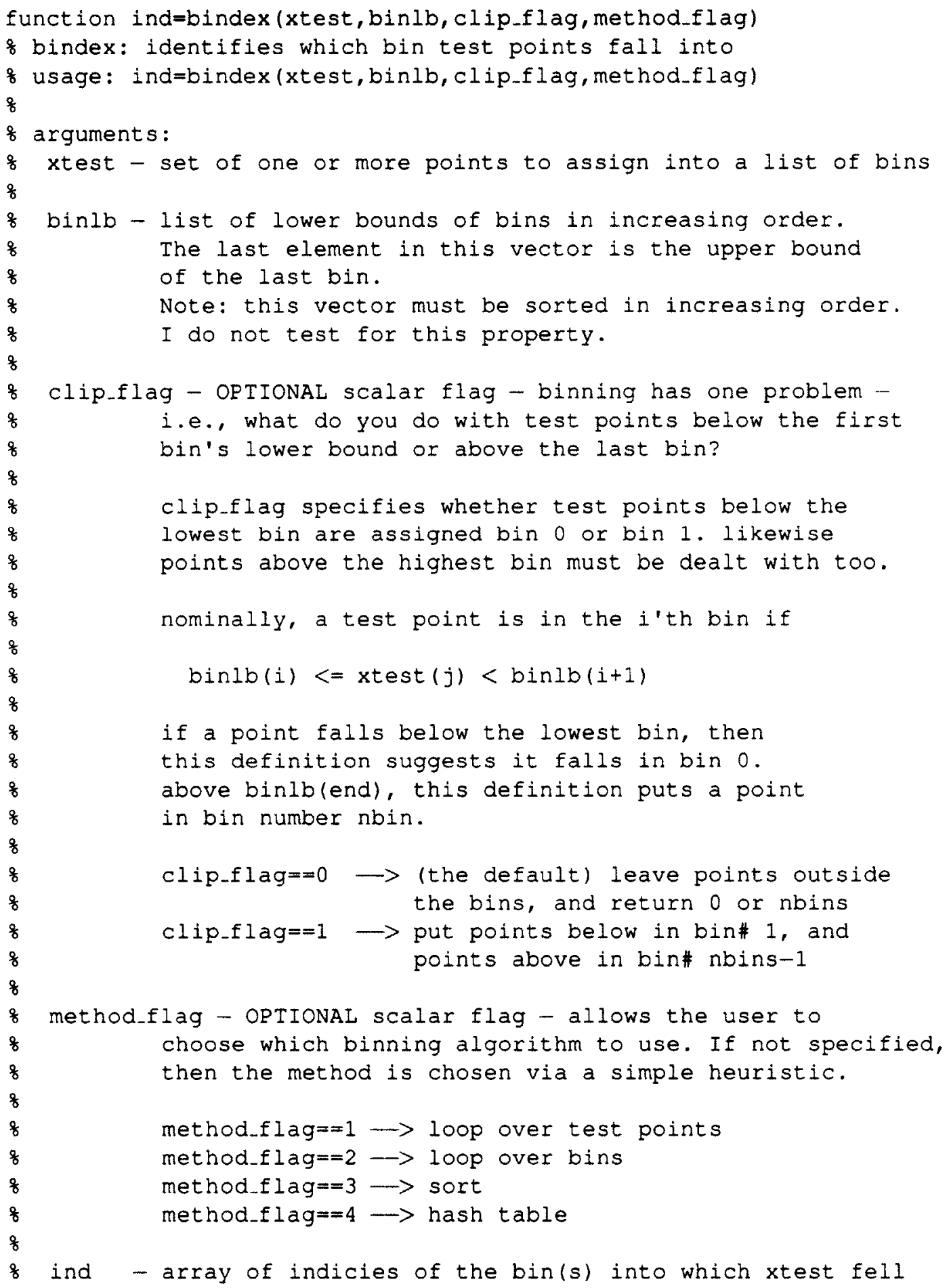

\& author: John R. D'Errico 
o email: woodchipslaworldnet.att.net

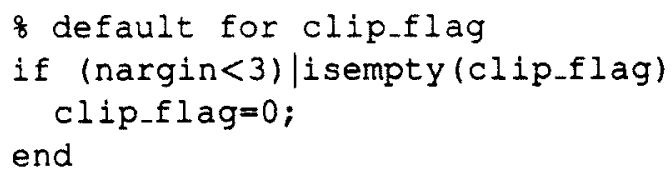




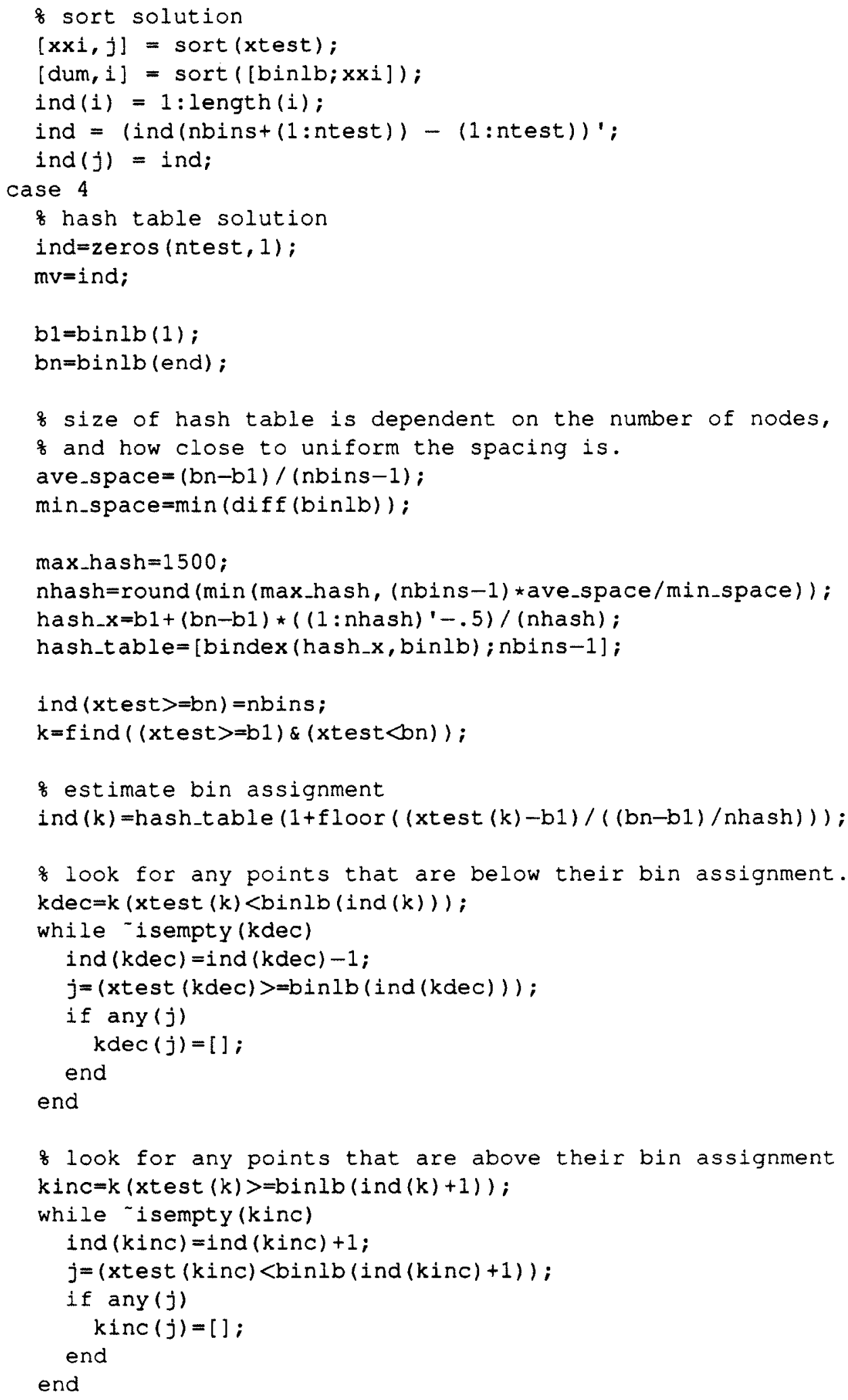


$\frac{8}{b}$ be nice and return indices as the same shape as xtest. ind=reshape (ind, sizeind);

\& do we return a bin index below 1 or above nbin-1?

if clip_flag ind $=\max (1, \min (n b i n s-1$, ind) );

end 


\section{Appendix B}

\section{Results Extra}

\section{B.1 Human vs. Linear Table}

\begin{tabular}{|c|c|c|c|c|}
\hline Patient & \multicolumn{4}{|c|}{ Pixel } \\
\hline & $\begin{array}{l}\text { Pixel 1 } \\
\text { LIP, UIP }\end{array}$ & $\begin{array}{l}\text { Pixel } 2 \\
\text { LIP, UIP }\end{array}$ & $\begin{array}{l}\text { Pixel } 3 \\
\text { LIP, UIP }\end{array}$ & $\begin{array}{l}\text { Pixel } 4 \\
\text { LIP, UIP }\end{array}$ \\
\hline 7 & $0.403-1.978,-0.118-2.058$ & $-1.122-1.466,1.365-1.41$ & $-1.693-1.878,-0.164-1.786$ & $-2.198-2.586,-0.807-2.457$ \\
\hline 8 & $1.629-1.355,-0.179-0.789$ & $0.375-0.955,0.930-1.186$ & $0.0970-1.969,0.602-1.259$ & $0.405-1.339,-1.899-0.972$ \\
\hline 11 & $2.992-3.858,-0.667-3.887$ & $0.735-2.321,-2.875-1.915$ & $2.453-3.406,-0.845-2.511$ & $-0.936-2.319,-1.47-2.56$ \\
\hline 12 & $-0.265-1.891,1.63-2.46$ & $0.782-1.125,-1.042-2.023$ & $2.029-2.742,1.696-2.462$ & $0.600-1.947,0.677-2.988$ \\
\hline 13 & $0.993-2.924,-5.043-2.744$ & $0.970-1.748,0.135-3.598$ & $-1.787-3.536,-2.954-3.446$ & $-0.537-3.022,-4.95-3.976$ \\
\hline 14 & $-2.369-3.314,-3.283-2.56$ & $-1.193-1.948,-0.529-2.336$ & $-2.965-3.206,-1.018-1.865$ & $-2.567-3.668,-2.168-2.056$ \\
\hline 15 & $-2.503-1.297,-1.621-2.271$ & $1.528-0.811,2.471-1.88$ & $-4,413-1.893,-3.90-1.801$ & $1.982-1.787,2.741-2.035$ \\
\hline 16 & $3.484-2.338,-3.406-2.47$ & $-0.194-3.428,3.238-3.199$ & $-0.716-2.271,-2.579-3.386$ & $-2.238-2.48,-3.059-2.18$ \\
\hline 17 & $-3.705-1.37,-2.872-1.872$ & $2.661-2.05,1.957-1.546$ & $-0.908-1.738,-0.0740-1.944$ & $2.009-1.178,2.085-2.49$ \\
\hline 18 & $2.223-1.014,2.527-2.163$ & $-2.026-1.749,-2.663-1.412$ & $-1.273-1.287,-2.507-0.882$ & $-2.779-1.347,-1.269-1.418$ \\
\hline 19 & $0.464-1.163,2.137-1.109$ & $2.368-2.332,2.19-1.905$ & $-0.457+2.232,0.100-2.039$ & $-3.178-4.36,0.311-2.917$ \\
\hline 20 & $0.335-1.768,1.812-1.976$ & $0.640-1.72,-0.0310-2.224$ & $-1.158-2.439,-0.808-2.304$ & $3.433-2.55,1.659-2.168$ \\
\hline 21 & $2.60-2.91,-0.170-3.009$ & $-7.337-3.735,-5.488-1.871$ & $-4.071-3.595,-4.755-1.844$ & $-3.03-2.621,0.430-2.072$ \\
\hline 23 & $2.161-1.756,1.472-1.776$ & $0.786-0.622,-0.884-2.752$ & $-2.363-1.632,-2.783-2.243$ & $-2.068-3.497,-1.249-4.299$ \\
\hline 25 & $-1.814-1.137,-0.761-2.261$ & $-0.820-2.194,-0.187-2.634$ & $-3.209-2.124,-0.913-1.86$ & $-0.435-3.462,-0.618-2.857$ \\
\hline 26 & $-6.077-3.807,-1.992-4.467$ & $-1.42-2.563,-0.915-1.535$ & $2.891-2.746,-0.316-3.74$ & $-3.583-2.891,-2.631-1.576$ \\
\hline 27 & $-1.099-0.856,1.285-2.064$ & $-1.609-2.204,-1.116-2.211$ & $-4.196-2.784,-0.678-2.272$ & $-2.554-3.207,-6.011-4.018$ \\
\hline 28 & $-0.384-1.544,-2.58-3.367$ & $0.472-1.664,-2.023-3.385$ & $-0.326-2.178,-3.242-1.778$ & $1.859-4.324,0.104-2.821$ \\
\hline 29 & $-2.112-2.213,-1.999-1.966$ & $-0.738-1.678,-1.851-1.161$ & $-2.402-1.513,-1.781-1.966$ & $0.589-1.482,0.347-1.619$ \\
\hline 30 & $2.137-1.317,2.025-2.21$ & $-0.161-1.316,-0.0100-1.569$ & $-1.339-1.062,-1.024-2.111$ & $-0.0870-2.02,0.804-2.223$ \\
\hline 32 & $1.711-1.91,2.553-1.668$ & $2.548-1.147,2.483-1.784$ & $-2.371-1.801,0.0570-1.757$ & $-0.301-1.694,-0.0200-1.833$ \\
\hline 39 & $0.0300-1.371,1.299-2.428$ & $-0.0350-1.957,1.285-1.33$ & $-2.401-1.925,-2.631-1.076$ & $-0.664-1.345,0.625-1.761$ \\
\hline 40 & $0.232-1.256,3.162-1.92$ & $-0.861-2.297,-0.0240-1.509$ & $-1.417-1.627,0.826-1.952$ & $2.137-2.651,1.249-1.788$ \\
\hline 41 & $-2.356-2.866,-0.775-2.418$ & $-0.800-1.23,3.532-2.084$ & $-1.216-1.90,-1.137-1.737$ & $0.632-1.295,1.342-2.212$ \\
\hline 42 & $-2.345-1.377,-2.935-1.895$ & $-0.628-1.301,1.488-1.938$ & $-1.657-1.328,-0.747-1.223$ & $-2.455-1.862,-3.475-1.576$ \\
\hline 43 & $-0.478-2.013,-0.517-2.34$ & 2.51-1.155,2.455-2.595 & $-1.173-3.231,-4.285-1.747$ & $-1.83-1.651,0.507-2.413$ \\
\hline Mean & $-0.278-1.946,-0.101-2.313$ & $0.0160-1.797,0.0240-2.038$ & $-1.507-2.232,-1.252-2.03$ & $-0.833-2.407,-0.620-2.357$ \\
\hline Median & $0.008-1.768,-0.0450-2.261$ & $0.153-1.748,0-1.915$ & $-1.499-2.124,-0.837-1.944$ & $-0.833-2.407,-0.601-2.212$ \\
\hline
\end{tabular}

Table B.1: Results from the comparison between the visual heuristics and the three piece linear methods. The table is formatted such that the first values indicate the median difference followed by it's standard deviation for the lower inflection points and then the median difference followed by it's standard deviation for the upper inflection point all for pixel 1. From here the pattern repeats for the last three pixels. 


\section{B.2 Visual Heuristics Pixel Locations}
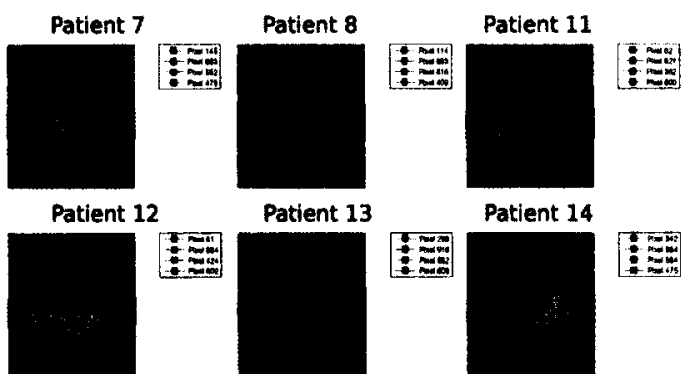

$8=$

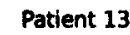

Patient 14



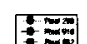

Patient 14

(15)

Patient 15

Patient 16

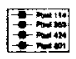
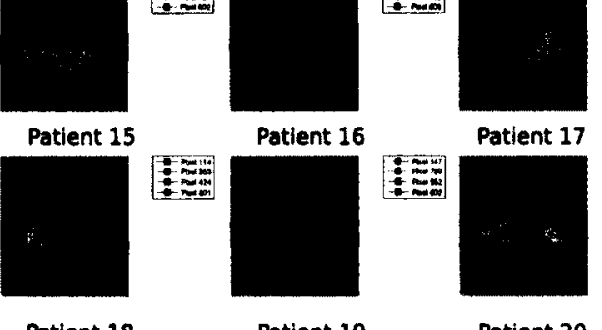

Patient 17

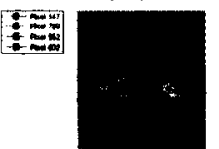

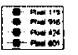

Patient 18

Patient 19

Patient 20


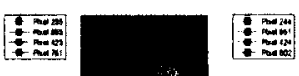

Patient 21

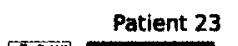

Patient 25

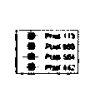
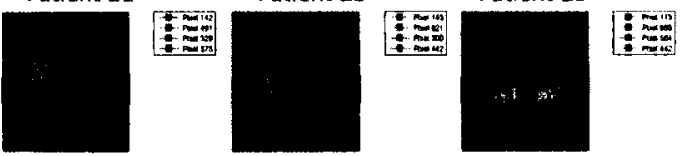

Patient 26
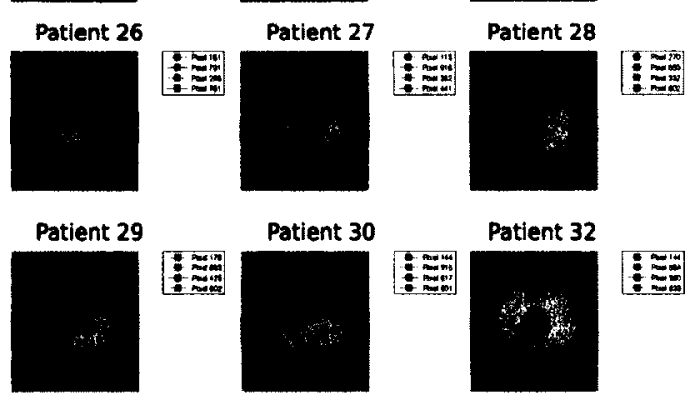

Patient 39

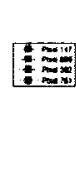

Patient 40

Patient 41
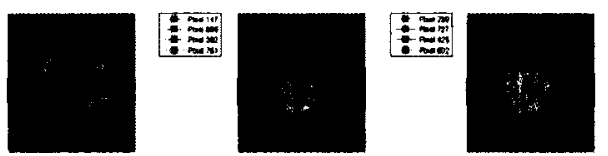

:

Patient 42

Patlent 43
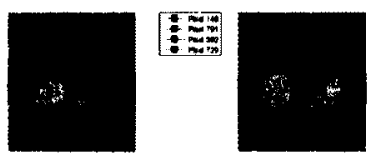

:

Figure B.1: Locations of the pixels used in the visual heuristic experiment. 


\section{References}

[Adler et al., 2012] Adler, A., Amato, M., Arnold, J., Bayford, R., Bodenstein, M., Böhm, S., Brown, B., Frerichs, I., Stenqvist, O., Weiler, N., and Wolf, G. (2012). Whither lung EIT: where are we, where do we want to go, and what do we need to get there? Submitted for publication to Journal of Applied Physiology.

[Adler et al., 1997] Adler, A., Amyot, R., Guardo, R., Bates, J. H. T., and Berthiaume, Y. (1997). Monitoring changes in lung air and liquid volumes with electrical impedance tomography. Journal of Applied Physiology, 83(5):1762-1767.

[Adler et al., 2009] Adler, A., Arnold, J. H., Bayford, R., Borsic, A., Brown, B., Dixon, P., Faes, T. J. C., Frerichs, I., Gagnon, H., Gärber, Y., Grychtol, B., Hahn, G., Lionheart, W. R. B., Malik, A., Patterson, R. P., Stocks, J., Tizzard, A., Weiler, N., and Wolf, G. K. (2009). GREIT: a unified approach to 2D linear EIT reconstruction of lung images. Physiological Measurement, 30(6):S35.

[Adler et al., 2011] Adler, A., Gaburro, R., and Lionheart, W. (2011). Handbook in Mathematical Methods in Imaging. XVIII. Springer, 1 edition.

[Adler and Guardo, 1996a] Adler, A. and Guardo, R. (1996a). Electrical impedance tomography: regularized imaging and contrast detection. Medical Imaging, IEEE Transactions on, 15(2):170-179. 
REFERENCES

[Adler and Guardo, 1996b] Adler, A. and Guardo, R. (1996b). Electrical impedance tomography: Regularized imaging and contrast detection. IEEE Transaction on Medical Imaging, 15(2):170-179.

[Adler et al., 1996] Adler, A., Guardo, R., and Berthiaume, Y. (1996). Impedance imaging of lung ventilation: do we need to account for chest expansion? Biomedical Engineering, IEEE Transactions on, 43(4):414-420.

[Adler and Lionheart, 2006] Adler, A. and Lionheart, W. R. B. (2006). Uses and abuses of EIDORS: an extensible software base for EIT. Physiological Measurement, 27(5):S25

[Albaiceta et al., 2004] Albaiceta, G. M., Taboada, F., Parra, D., Luyando, L. H., Calvo, J., Menendez, R., and Otero, J. (2004). Tomographic study of the inflection points of the pressure-volume curve in acute lung injury. Am. J. Respir. Crit. Care Med., 170(10):1066-1072.

[Allud and Martin, 1977] Allud, L. A. and Martin, M. H. (1977). Schlumberger: The history of a technique. John Wiley and Sons, New York.

[Amato et al., 1995] Amato, M. B., Barbas, C. S., Medeiros, D. M., de P Schettino, G., Filho, G. L., Kairalla, R. A., Deheinzelin, D., Morais, C., de O Fernandes, E., Takagaki, T. Y., and et al (1995). Beneficial effects of the "open lung approach" with low distending pressures in acute respiratory distress syndrome. A prospective randomized study on mechanical ventilation. Am J Respir Crit Care Med., $152: 1835-1846$.

[Amato et al., 1998] Amato, M. B. P., Barbas, C. S. V., Medeiros, D. M., Magaldi, R. B., Schettino, G. P., Lorenzi-Filho, G., Kairalla, R. A., Deheinzelin, D., Munoz, C., Oliveira, R., Takagaki, T. Y., and Carvalho, C. R. R. (1998). Effect 
of a protective-ventilation strategy on mortality in the acute respiratory distress syndrome. New England Journal of Medicine, 338(6):347-354.

[Andersen, 2008] Andersen, M. H. (2008). Investigation of lung mechanics using CT scan analysis, a mathematical model and the static pressure volume curve. Master's thesis, Aalborg University.

[Aster et al., 2004] Aster, R. C., Borchers, B., and Thurber, C. (2004). Parameter Estimation and Inverse Problems, chapter 2.2. Elsevier Academic Press.

[Bernard et al., 1994] Bernard, G., Artigas, A., Brigham, K., Carlet, J., Falke, K., Hudson, L., Lamy, M., Legall, J., Morris, A., and Spragg, R. (1994). The americaneuropean consensus conference on ARDS. definitions, mechanisms, relevant outcomes, and clinical trial coordination. Am. J. Respir. Crit. Care Med., 149(3):818824.

[Bigatello et al., 1999] Bigatello, William, H., and Antonio, P. (1999). Ventilatory management of severe acute respiratory failure for Y2K. Anesthesiology, 91(6).

[Bindslev et al., 1980] Bindslev, L., Hedenstierna, G., Santesson, J., Norlander, O., and Gram, I. (1980). Airway closure during anaesthesia, and its prevention by positive end expiratory pressure. Acta Anaesthesiologica Scandinavica, 24(3):199205.

[Borcea, 2002] Borcea, L. (2002). Electrical impedance tomography. Inverse Problems, 18(6):R99.

[Borges et al., 2006] Borges, J. B., Okamoto, V. N., Matos, G. F. J., Caramez, M. P. R., Arantes, P. R., Barros, F., Souza, C. E., Victorino, J. A., Kacmarek, R. M., Barbas, C. S. V., Carvalho, C. R. R., and Amato, M. B. P. (2006). Reversibility 
of lung collapse and hypoxemia in early acute respiratory distress syndrome. Am. J. Respir. Crit. Care Med., 174(3):268-278.

[Boyle, 2010] Boyle, A. J. S. (2010). The effect of shape deformation on twodimensional electrical impedance tomography. Master's thesis, Carleton University.

[Brunet et al., 1995] Brunet, F., Jeanbourquin, D., Monchi, M., Mira, J., Fierobe, L., Armaganidis, A., Renaud, B., Belghith, M., Nouira, S., and Dhainaut, J. (1995). Should mechanical ventilation be optimized to blood gases, lung mechanics, or thoracic CT scan? Am. J. Respir. Crit. Care Med., 152(2):524-530.

[Carlton et al., 1990] Carlton, D. P., Cummings, J. J., Scheerer, R. G., Poulain, F. R., and Bland, R. D. (1990). Lung overexpansion increases pulmonary microvascular protein permeability in young lambs. Journal of Applied Physiology, 69(2):577-583.

[Colebatch et al., 1979] Colebatch, H. J., Ng, C. K., and Nikov, N. (1979). Use of an exponential function for elastic recoil. Journal of Applied Physiology, 46(2):387393.

[Costa et al., 2009] Costa, E., Borges, J., Melo, A., Suarez-Sipmann, F., Toufen, C., Bohm, S., and Amato, M. (2009). Bedside estimation of recruitable alveolar collapse and hyperdistension by electrical impedance tomography. Intensive Care Medicine, 35:1132-1137.

[Coulombé et al., 2005] Coulombé, N., Gagnon, H., Marquis, F., Skrobik, Y., and Guardo, R. (2005). A parametric model of the relationship between EIT and total lung volume. Physiological Measurement, 26(4):401.

[Dambrosio et al., 1997] Dambrosio, M., Roupie, E., Mollet, J.-J., Anglade, M.-C., Vasile, N., Lemaire, F., and Brochard, L. (1997). Effects of positive end-expiratory 
pressure and different tidal volumes on alveolar recruitment and hyperinflation. Anesthesiology, 87:495-503.

[de Abreu et al., 2003] de Abreu, M. G., Heintz, M., Heller, A., Szechenyi, R., Albrecht, D. M., and Koch, T. (2003). One-lung ventilation with high tidal volumes and zero positive end-expiratory pressure is injurious in the isolated rabbit lung model. Anesthesia and Analgesia, 96(1):220-228.

[D'Errico, 2011] D'Errico, J. (2011). MATLAB central - file exchange. http://www. mathworks.com/matlabcentral/fileexchange/authors/679.

[Dreyfus and Saumon, 1998] Dreyfus, D. and Saumon, G. (1998). Ventilator-induced lung injury. lessons from experimental studies. Am. J. Respir. Crit. Care Med, S31-43.

[Dreyfuss and Saumon, 1993] Dreyfuss, D. and Saumon, G. (1993). Role of tital volume, FRC, and end-inspiratory volume in the deveolopment of pulmonary edema following mechanical ventilation. Am Rev Respir Dis, 148:1194-1203.

[EIDORS, 2011] EIDORS (2011). EIDORS: Electrical impedance tomography and diffuse optical tomography reconstruction software. http://eidors3d. sourceforge.net/.

[Faes et al., 2006] Faes, T. J. C., van Genderingen, H. R., and Noordegraaf, A. V. (2006). Some reflections on the EIT conference (London, UK, 22-24 June 2005). Physiological Measurement, 27(5).

[Frerichs et al., 2003] Frerichs, I., Dargaville, P., Dudykevych, T., and Rimensberger, P. (2003). Electrical impedance tomography: a method for monitoring regional lung aeration and tidal volume distribution? Intensive Care Medicine, 29. 
[Frerichs et al., 2002] Frerichs, I., Hinz, J., Herrmann, P., Weisser, G., Hahn, G., Dudykevych, T., Quintel, M., and Hellige, G. (2002). Detection of local lung air content by electrical impedance tomography compared with electron beam CT. Journal of Applied Physiology, 93(2):660-666.

[Fu et al., 1992] Fu, Z., Costello, M. L., Tsukimoto, K., Prediletto, R., Elliott, A. R., Mathieu-Costello, O., and West, J. B. (1992). High lung volume increases stress failure in pulmonary capillaries. Journal of Applied Physiology, 73(1):123-133.

[Gattinoni et al., 1987] Gattinoni, L., Pesenti, A., Avalli, L., Rossi, F., and Bombino, M. (1987). Pressure-volume curve of total respiratory system in acute respiratory failure: Computed tomographic scan study. Am. J. Respir. Crit. Care Med., 136(3):730-736.

[Gattinoni et al., 1984] Gattinoni, L., Pesenti, A., Caspani; N.; Pelizzola, A., Mascheroni, D., Marcolin, R., Iapichino, G., Langer, M., Agostoni, A., Kolobow, T., Melrose, D., and Damia, G. (1984). The role of total static lung compliance in the management of severe ARDS unresponsive to conventional treatment. Intensive Care Medicine, 10:121-126.

[Genderingen et al., 2004] Genderingen, H. R. V., Van Vught, A. J., and Jansen, J. R. C. (2004). Regional lung volume during high-frequency oscillatory ventilation by electrical impedance tomography. Critical Care Medicine, 32(3):787-794.

[Graham, 2007] Graham, B. M. (2007). Enhancements in Electrical Impedance Tomography (EIT) Image Reconstruction for 3D Lung Imaging. $\mathrm{PhD}$ thesis, University of Ottawa. 
[Greenleaf et al., 2003] Greenleaf, A., Lassas, M., and Uhlmann, G. (2003). Anisotropic conductivities that cannot be detected by EIT. Physiological Measurement, 24(2):413.

[Grychtol et al., 2010] Grychtol, B., Wolf, G. K., Adler, A., and Arnold, J. H. (2010). Towards lung EIT image segmentation: automatic classification of lung tissue state from analysis of EIT monitored recruitment manoeuvres. Physiological Measurement, 31(8):S31.

[Grychtol et al., 2009] Grychtol, B., Wolf, G. K., and Arnold, J. H. (2009). Differences in regional pulmonary pressure impedance curves before and after lung injury assessed with a novel algorithm. Physiological Measurement, 30(6):S137.

[Hahn et al., 1996] Hahn, G., Frerichs, I., Kleyer, M., and Hellige, G. (1996). Local mechanics of the lung tissue determined by functional EIT. Physiological Measurement, 17(4A):A159.

[Harris, 2005] Harris, R. S. (2005). Pressure-volume curves of the respiratory system. Respiratory Care, 50(1):78-99.

[Harris et al., 2000] Harris, R. S., Hess, D. R., and Venegas, J. G. (2000). An objective analysis of the pressure-volume curve in the acute respiratory distress syndrome. Am. J. Respir. Crit. Care Med., 161(2):432-439.

[Hasan, 2010] Hasan, A. (2010). Understanding Mechanical Ventilation A Practical Handbook. Springer-Verlab London Limited.

[Hickling, 1998] Hickling, K. G. (1998). The pressure-volume curve is greatly modified by recruitment . A mathematical model of ARDS lungs. Am. J. Respir. Crit. Care Med., 158(1):194-202. 
[Hinz et al., 2003a] Hinz, Hahn, Neumann, Sydow, Mohrenweiser, Hellige, and Burchardi (2003a). End-expiratory lung impedance change enables bedside monitoring of end-expiratory lung volume change. Intensive Care Medicine, 29:37-43.

[Hinz et al., 2006] Hinz, J., Moerer, O., Neumann, P., Dudykevych, T., Frerichs, I., Hellige, G., and Quintel, M. (2006). Regional pulmonary pressure volume curves in mechanically ventilated patients with acute respiratory failure measured by electrical impedance tomography. Acta Anaesthesiologica, 50(3):331-339.

[Hinz et al., 2003b] Hinz, J., Neumann, P., Dudykevych, T., Andersson, L. G., Wrigge, H., Burchardi, H., and Hedenstierna, G. (2003b). Regional ventilation by electrical impedance tomography: a comparison with ventilation scintigraphy in pigs. Chest, 124(1):314-322.

[Holder, 2004] Holder, D. (2004). Electrical Impedance Tomography: Methods, History, Applications. Institute of Physics Publishing.

[Hudson, 1989] Hudson, L. D. (1989). Survival data in patients with acute and chronic lung disease requiring mechanical ventilation. Am. J. Respir. Crit. Care Med., 140.

[Ioannidis, 2005] Ioannidis, J. P. A. (2005). Why most published research findings are false. PLoS Med, 2(8):e124.

[Kunst et al., 2000] Kunst, P. W., Böhm, S. H., Anda, G. V. D., Amato, M. B., Lachmann, B., Postmus, P. E., and De Vries, P. M. (2000). Regional pressure volume curves by electrical impedance tomography in a model of acute lung injury. Critical Care Medicine, 28(1):178-183. 
[Kunst et al., 1999] Kunst, P. W. A., de Vries, P. M. J. M., Postmus, P. E., and Bakker, J. (1999). Evaluation of electrical impedance tomography in the measurement of PEEP-induced changes in lung volume. Chest, 115(4):1102-1106.

[Lu et al., 1999] Lu, Q., Vieira, S. R., Richecoeur, J., Puybasset, L., Kalfon, P., Coriat, P., and Rouby, J.-J. (1999). A simple automated method for measuring pressure-volume curves during mechanical ventilation. Am. J. Respir. Crit. Care Med., 159(1):275-282.

[Luepschen et al., 2007] Luepschen, H., Meier, T., Grossherr, M., Leibecke, T., Karsten, J., and Leonhardt, S. (2007). Protective ventilation using electrical impedance tomography. Physiological Measurement, 28(7):S247.

[Maggiore et al., 2003] Maggiore, S. M., Richard, J.-C., and Brochard, L. (2003). What has been learnt from $\mathrm{P} / \mathrm{V}$ curves in patients with acute lung injury/acute respiratory distress syndrome. European Respiratory Journal, 22(42 suppl):22s-26s.

[Marini, 2001] Marini, J. J. (2001). Ventilator-induced airway dysfunction? Am. J. Respir. Crit. Care Med., 163(4):806-807.

[Martin-Lefevre et al., 2001] Martin-Lefevre, L., Ricard, J.-D., Roupie, E., Dreyfuss, D., and Saumon, G. (2001). Significance of the changes in the respiratory system pressure-volume curve during acute lung injury in rats. Am. J. Respir. Crit. Care Med., 164(4):627-632.

[Massad et al., 2008] Massad, E., Ortega, N. R. S., de Barros, L. C., and Struchiner, C. J. (2008). Fuzzy Logic in Action: Applications in Epidemiology and Beyond, volume 232 of Studies in Fuzziness and Soft Computing. Springer. 
[Matamis et al., 1984a] Matamis, D., Lemaire, F., Harf, A., Brun-Buisson, C., Ansquer, J. C., and Atlan, G. (1984a). Total respiratory pressure-volume curves in the adult respiratory distress syndrome. Chest, 86(1):58-66.

[Matamis et al., 1984b] Matamis, D., Lemaire, F., Harf, A., Brun-Buisson, C., Ansquer, J. C., and Atlan, G. (1984b). Total respiratory pressure-volume curves in the adult respiratory distress syndrome. Chest, 86(1):58-66.

[Mead et al., 1970] Mead, J., Takishima, T., and Leith, D. (1970). Stress distribution in lungs: a model of pulmonary elasticity. Journal of Applied Physiology, 28(5):596608.

[Meeson et al., 1996] Meeson, S., Blott, B. H., and Killingback, A. L. T. (1996). EIT data noise evaluation in the clinical environment. Physiological Measurement, $17(4 \mathrm{~A}): \mathrm{A} 33$.

[Meier et al., 2008] Meier, T., Luepschen, H., Karsten, J., Leibecke, T., Groherr, M., Gehring, H., and Leonhardt, S. (2008). Assessment of regional lung recruitment and derecruitment during a PEEP trial based on electrical impedance tomography. Intensive Care Medicine, 34:543-550.

[Mendel, 1995] Mendel, J. M. (1995). Fuzzy logic systems for engineering: a tutorial. Proceedings of the IEEE, 83(3):345-377.

[Mendel, 2001] Mendel, J. M. (2001). Uncertain rule-based fuzzy logic system: introduction and new directions. Prentice-Hall PTR, $1^{\text {st }}$ edition.

[Merriam-Webster, 2010] Merriam-Webster (2010). Medline plus - trusted health information for you. http://ww.nlm.nih.gov/medlineplus/mplusdictionary. html. 
[Muscedere et al., 1994] Muscedere, J., Mullen, J., Gan, K., and Slutsky, A. (1994). Tidal ventilation at low airway pressures can augment lung injury. Am. J. Respir. Crit. Care Med., 149(5):1327-1334.

[Neligan, 2006] Neligan, P. (2006). Pulmonary CCM tutorials. http://www. ccmtutorials. com/index.htm.

[Papadakis and Lachmann, 2007] Papadakis, P. J. and Lachmann, B. (2007). Mechanical Ventilation: Clincical Applications and Pathophysiology. Saunders Elsevier.

[Polydorides and Lionheart, 2002] Polydorides, N. and Lionheart, W. R. B. (2002). A matlab toolkit for three-dimensional electrical impedance tomography: a contribution to the electrical impedance and diffuse optical reconstruction software project. Measurement Science and Technology, 13(12):1871.

[Pulletz et al., 2011] Pulletz, S., Adler, A., Kott, M., Elke, G., Gawelczyk, B., Schädler, D., Zick, G., Weiler, N., and Frerichs, I. (2011). Regional lung opening and closing pressures in patients with acute lung injury. Journal of Critical Care, (0):-.

[Pulletz et al., 2006] Pulletz, S., van Genderingen, H. R., Schmitz, G., Zick, G., Schädler, D., Scholz, J., Weiler, N., and Frerichs, I. (2006). Comparison of different methods to define regions of interest for evaluation of regional lung ventilation by EIT. Physiological Measurement, 27(5):S115.

[Ranieri et al., 1997] Ranieri, V. M., Brienza, N., Santostasi, S., Puntillo, F., Mascia, L., Vitale, N., Giuliani, R., Memeo, V., Bruno, F., Fiore, T., Brienza, A., and Slutsky, A. S. (1997). Impairment of lung and chest wall mechanics in patients 
with acute respiratory distress syndrome . role of abdominal distension. Am. J. Respir. Crit. Care Med., 156(4):1082-1091.

[Ranieri et al., 1999] Ranieri, V. M., Suter, P. M., Tortorella, C., Tullio, R. D., Dayer, J. M., Brienza, A., Bruno, F., and Slutsky, A. S. (1999). Effect of mechanical ventilation on inflammatory mediators in patients with acute respiratory distress syndrome. JAMA: The Journal of the American Medical Association, 282(1):54-61.

[Ranieri et al., 2000] Ranieri, V. M., Zhang, H., Mascia, L., Aubin, M., Lin, C. Y., Mullen, J. B., Grasso, S., Binnie, M., Volgyesi, G. A., Eng, P., and et al. (2000). Pressure-time curve predicts minimally injurious ventilatory strategy in an isolated rat lung model. Anesthesiology, 93(5):1320-1328.

[Rawlins et al., 2003] Rawlins, R., Brown, K. M., Carr, C. S., and Cameron, C. R. (2003). Life threatening haemorrhage after anterior needle aspiration of pneumothoraces. A role for lateral needle aspiration in emergency decompression of spontaneous pneumothorax. Emergency Medicine Journal, 20(4):383-384.

[Rossi et al., 2008] Rossi, F. S. C. M., Mascaretti, R. S., Haddad, L. B., Freddi, N. A., Mauad, T., and Rebello (2008). Utilization of the lower inflection point of the pressure-volume curve results in protective conventional ventilation comparable to high frequency oscillatory ventilation in an animal model of acute respiratory distress syndrome. Clinics, 63:237-244.

[Rouby et al., 2002] Rouby, J. J., Lu, Q., and Goldstein, I. (2002). Selecting the right level of positive end-expiratory pressure in patients with acute respiratory distress syndrome. Am. J. Respir. Crit. Care Med., 165(8):1182-1186. 
[Salazar and Knowles, 1964] Salazar, E. and Knowles, J. H. (1964). An analysis of pressure-volume characteristics of the lungs. Journal of Applied Physiology, 19(1):97-104.

[Schraufnagel, 2010] Schraufnagel, D. (2010). Breathing in America: Diseases, Progress, and Hope. American Thoracic Society.

[Sivanandam et al., 2006] Sivanandam, S. N., Sumathi, S., and Deepa, S. N. (2006). Introduction to Fuzzy Logic using MATLAB. Springer-Verlag New York, Inc., Secaucus, NJ, USA.

[Slutsky, 1994] Slutsky, A. (1994). Consensus conference on mechanical ventilation january 28 30, 1993 at northbrook, illinois, USA. Intensive Care Medicine, 20:6479.

[Takeuchi et al., 2001] Takeuchi, M., Sedeek, K. A., Suchodolski, G. P. P. S. K., and Kacmarek, R. M. (2001). Peak pressure during volume history and pressure-volume curve measurement affects analysis. Am. J. Respir. Crit. Care Med., 164(7):12251230.

[Takeuchi et al., 2002] Takeuchi, R. M., Sven, G., Marisa, D., Motomu, S., Dean, H., B, A. M., and Kacmarek (2002). Set positive end-expiratory pressure during protective ventilation affects lung injury. Anesthesiology, 63:682-692.

[Tanaka, Harki and Neli Regina Siqueira Ortega and Mauricio Stanzione Galizia and João Batist Tanaka, Harki and Neli Regina Siqueira Ortega and Mauricio Stanzione Galizia and João Batista Borges and Amato, M. B. P. (2008). Fuzzy modeling of electrical impedance tomography images of the lungs. Clinics, 63:363-370. 
[Todd, 2010] Todd, R. B. (2010). Volume 4 of The Cyclopdia of Anatomy and Physiology. Sherwood, Gilbert, and Piper,.

[Tremblay et al., 1997] Tremblay, L., Valenza, F., Ribeiro, S. P., Li, J., and Slutsky, A. S. (1997). Injurious ventilatory strategies increase cytokines and c-fos m-RNA expression in an isolated rat lung model. The Journal of Clinical Investigation, 99:944-952.

[Tsuno et al., 1990] Tsuno, K., Prato, P., and Kolobow, T. (1990). Acute lung injury from mechanical ventilation at moderately high airway pressures. Journal of Applied Physiology, 69(3):956-961.

[Venegas et al., 1998] Venegas, J. G., Harris, R. S., and Simon, B. A. (1998). A comprehensive equation for the pulmonary pressure-volume curve. Journal of Applied Physiology, 84(1):389-395.

[Victorino et al., 2004] Victorino, J. A., Borges, J. B., Okamoto, V. N., Matos, G. F. J., Tucci, M. R., Caramez, M. P. R., Tanaka, H., Sipmann, F. S., Santos, D. C. B., Barbas, C. S. V., Carvalho, C. R. R., and Amato, M. B. P. (2004). Imbalances in regional lung ventilation: A validation study on electrical impedance tomography. Am. J. Respir. Crit. Care Med., 169(7):791-800.

[Vieillard-Baron and Jardin, 2002] Vieillard-Baron, A. and Jardin, F. (2002). Right level of positive end-expiratory pressure in acute respiratory distress syndrome. $\mathrm{Am}$ J Respir Crit Care Med, 167:1576-1577.

[Webster, 2009] Webster, J. G. (2009). Medical Instrumentation Application and Design, 4th Edition. Wiley. 
[Wolf et al., 2010] Wolf, G. K., Grychtol, B., Frerichs, I., Zurakowski, D., and Arnold, J. H. (2010). Regional lung volume changes during high-frequency oscillatory ventilation. 11(5):610-615.

[Wu and Mendel, 2011] Wu, D. and Mendel, J. M. (2011). Linguistic summarization using IFTHEN rules and interval type-2 fuzzy sets. Fuzzy Systems, IEEE Transactions on, 19(1):136-151.

[Yorkey and Webster, 1987] Yorkey, T. J. and Webster, J. G. (1987). A comparison of impedance tomographic reconstruction algorithms. Clinical Physics and Physiological Measurement, 8(4A):55.

[Zhang and Patterson, 2005] Zhang, J. and Patterson, R. P. (2005). EIT images of ventilation: what contributes to the resistivity changes? Physiological Measurement, 26(2):S81. 